\title{
Amplitudes, observables, and classical scattering
}

\author{
David A. Kosower, ${ }^{a}$ Ben Maybee ${ }^{b}$ and Donal O'Connell ${ }^{b}$ \\ ${ }^{a}$ Institut de Physique Théorique, CEA, CNRS, Université Paris-Saclay, \\ F-91191 Gif-sur-Yvette cedex, France \\ ${ }^{b}$ Higgs Centre for Theoretical Physics, School of Physics and Astronomy, \\ The University of Edinburgh, \\ Edinburgh EH9 3JZ, Scotland, U.K. \\ E-mail: David.Kosower@cea.fr, b.maybee@ed.ac.uk, \\ donal@staffmail.ed.ac.uk
}

AbSTRACT: We present a formalism for computing classically measurable quantities directly from on-shell quantum scattering amplitudes. We discuss the ingredients needed for obtaining the classical result, and show how to set up the calculation to derive the result efficiently. We do this without specializing to a specific theory. We study in detail two examples in electrodynamics: the momentum transfer in spinless scattering to next-to-leading order, and the momentum radiated to leading order.

Keywords: Scattering Amplitudes, Classical Theories of Gravity

ArXiv EPrint: 1811.10950 


\section{Contents}

1 Introduction 1

2 Restoring $\hbar+3$

3 Impulse and radiated momentum in quantum field theory 5

3.1 The incoming state 5

$\begin{array}{lll}3.2 & \text { The impulse on a particle } & 7\end{array}$

3.3 Impulse in terms of amplitudes 8

$\begin{array}{ll}3.4 & \text { The momentum radiated during a collision } \\ \end{array}$

$\begin{array}{ll}3.5 \text { Conservation of momentum } & 13\end{array}$

4 Classical point particles $\quad \mathbf{1 5}$

$\begin{array}{lll}4.1 & \text { Wavefunctions } & 15\end{array}$

$\begin{array}{lll}4.2 & \text { An example wavefunction } & 20\end{array}$

4.3 Classical impulse 22

4.4 Classical radiation 24

$\begin{array}{ll}4.5 & \text { Conservation of momentum } \\ \end{array}$

5 Examples $\quad \mathbf{2 7}$

5.1 Leading-order electromagnetic impulse 27

$\begin{array}{ll}5.2 \text { Next-to-leading order impulse } & 29\end{array}$

$\begin{array}{lll}5.2 .1 & \text { Purely quantum contributions } & 30\end{array}$

$\begin{array}{lll}5.2 .2 & \text { Triangles } & 31\end{array}$

$\begin{array}{lll}5.2 .3 & \text { Boxes } & 32\end{array}$

5.2.4 Cut box 34

$\begin{array}{ll}\text { 5.2.5 Combining contributions } & 35\end{array}$

$\begin{array}{lll}5.2 .6 & \text { On-shell cross check } & 37\end{array}$

$\begin{array}{lll}5.3 \text { Radiation } & 38\end{array}$

5.4 Momentum conservation and radiation reaction 40

6 Classical calculations $\quad 42$

6.1 The classical electromagnetic impulse $\quad 42$

6.1.1 Leading order 43

6.1.2 Next-to-leading order 44

$\begin{array}{ll}6.2 & \text { Classical radiated momentum }\end{array}$

$\begin{array}{lll}\text { 6.2.1 General expressions } & 46\end{array}$

6.2.2 Application at leading order 48

6.3 Momentum conservation and the radiation reaction force 49

$\begin{array}{lll}7 & \text { Discussion and conclusions } & 51\end{array}$ 


\section{Introduction}

The dawn of gravitational-wave astronomy, heralded by the binary black-hole and neutronstar mergers detected by the LIGO collaboration [1-5], has spawned interest in new techniques for solving the two-body problem in gravity and generating the theoretical waveforms required [6] for event detection as well as parameter extraction from observed mergers. Such techniques would complement methods based on the 'traditional' Arnowitt-Deser-Misner Hamiltonian formalism [7-11], direct post-Newtonian solutions in harmonic gauge [12], long-established effective-one-body (EOB) methods [13-16], numerical-relativity approaches $[17,18]$, and the effective-field theory approach pioneered by Goldberger and Rothstein [19-68].

Our broader interest is in exploring the application of modern scattering-amplitudes techniques to this question. Indeed, amplitudes have already been applied successfully to understand aspects of the general relativistic two-body problem, notably to computing the potential between two masses [69-76]. The relevance of a scattering amplitude in particular, a loop amplitude - to the classical potential was understood in earlier work on gravity as an effective field theory [77-82], and was emphasised by Donoghue and Holstein [83]. This connection will be important for us below. More recently, Damour has emphasised that methods based on scattering amplitudes are relevant to the EOB formalism $[84,85]$.

An important insight arising from the study of scattering amplitudes is that gravitational amplitudes are simpler than one would expect, and in particular are closely connected to the amplitudes of Yang-Mills theory. This connection is called the double copy, because gravitational amplitudes are obtained as a product of two Yang-Mills quantities. One can implement this double copy in a variety of ways: the original statement, by Kawai, Lewellen and Tye [86] presents a tree-level gravitational amplitude as a sum over terms, each of which is a product of two tree-level color-ordered Yang-Mills amplitudes (multiplied by appropriate Mandelstam invariants). More recently, Bern, Carrasco and Johansson $[87,88]$ demonstrated that the double copy can be understood very simply in terms of a diagrammatic expansion of a scattering amplitude: the gravitational numerators are simply the square of the kinematic numerators in Yang-Mills theory, once a property known as colour-kinematics duality is imposed on the numerators. These advances were particularly exciting as they lead to a clear generalisation to loop level. The work of BCJ 
suggests that gravity may be simpler than it seems, and also more closely connected to Yang-Mills theory than one would guess after inspecting their Lagrangians. We may hope that these insights will be relevant to the real-world physics of gravitational waves.

The double copy indeed connects classical solutions of Yang-Mills theory and gravity. In particular, point charges in Yang-Mills theory map to point sources in gravity [89-92]. This holds true to all orders, even for accelerating particles [90, 93], and we know that the classical radiation emitted by accelerating particles does indeed double copy from Yang-Mills theory to gravity [90, 91, 94, 95], even for a particle moving in an arbitrary manner [91, 95], at least to the first two orders of perturbation theory. There are also indications that the double copy can encompass bound states [96, 97] and perhaps spinning particles $[98,99]$. These observations suggest that scattering-amplitudes methods, which naturally incorporate spin, should apply to the classical gravitational physics of spinning matter [100].

In addition to offering us the double copy, the techniques of scattering amplitudes [101] or an analysis of soft limits [102-104] may help to simplify the computation of physical waveforms relevant for gravitational wave observatories. First, though, we must understand systematically how to extract the classical result using on-shell quantum-mechanical scattering amplitudes in order to take full advantage of amplitude methods in the gravitationalwave problem.

The present article is a step in this direction. We focus directly on physical observables, extracting the classical values from a fully relativistic quantum-mechanical computation. We examine two particular observables. The first is the change in momentum during a scattering event, both with and without accompanying radiation. The second is the radiated momentum during the event. We shall use them as a laboratory to explore certain conceptual and practical issues in approaching the classical limit. Our formalism applies to both electrodynamics and gravity. We will work out in detail explicit examples in electrodynamics, but many of the issues we explore also arise in the gravitational case. For simplicity, we restrict to spinless scattering in this article.

Our two observables are not completely independent. Indeed the relation between them goes to the heart of one of the difficulties in traditional approaches to classical field theory with point sources. In two-particle scattering in classical electrodynamics, for example, momentum is transferred from one particle to the other via the electromagnetic field, as described by the Lorentz force. But the energy-momentum lost by point particles to radiation is not accounted for by the Lorentz force. Conservation of momentum is restored by taking into account an additional force, the Abraham-Lorentz-Dirac (ALD) force [105-110], see e.g. refs. [111-116] for more recent treatments. Inclusion of this radiation reaction force is not without cost: rather, it leads to the celebrated issues of runaway solutions or causality violations in the classical electrodynamics of point sources.

The quantum-mechanical description of charged-particle scattering should cure these ills. Indeed we will see explicitly that a quantum-mechanical description will conserve energy and momentum in particle scattering automatically.

In the next section, we begin by describing where the factors of $\hbar$ appear in scattering amplitudes. As we will see it is straightforward to make these factors explicit, but 
nevertheless there are aspects of extracting the classical result that remain obscure. This motivates the following section, where we give a formal definition of the momentum transfer to a particle in quantum field theory and of the expectation value of the momentum emitted in radiation during a scattering event. We also give expressions for these observables in terms of on-shell scattering amplitudes. In section 4, we discuss the quantum wavefunctions suitable for studying classical point particles, and derive simplified formulæ for our observables. In section 5 we apply our formalism in electrodynamics to compute the two classical observables, the momentum transfer and the radiated momentum, from scattering amplitudes. In section 6, we perform the corresponding classical calculations, and compare the results with those obtained from quantum field theory. Section 7 contains a discussion of our results and our conclusions. In the appendices, we provide some details on our conventions and some of the integrals we used, as well as dwelling in more detail on radiation in the classical theory.

\section{Restoring $\hbar$}

A straightforward and pragmatic approach to restoring all factors of $\hbar$ in an expression is dimensional analysis. ${ }^{1}$ We will continue to use relativistically natural units, with $c=1$. We denote the dimensions of mass and length by $[M]$ and $[L]$, respectively.

We may choose the dimensions of an $n$-point scattering amplitude in four dimensions to be $[M]^{4-n}$ even when $\hbar \neq 1$. This is consistent with choosing the dimensions of creation and annihilation operators so that,

$$
\left[a_{p}, a_{p^{\prime}}^{\dagger}\right]=(2 \pi)^{3} \delta^{(3)}\left(\boldsymbol{p}-\boldsymbol{p}^{\prime}\right)
$$

where bold symbols (here $\boldsymbol{p}$ and $\boldsymbol{p}^{\prime}$ ) denote spatial three-vectors. We define single-particle states by,

$$
|p\rangle=\sqrt{2 E_{p}} a_{p}^{\dagger}|0\rangle .
$$

The dimension of $|p\rangle$ is thus $[M]^{-1}$. (The vacuum state is taken to be dimensionless.) We further define $n$-particle asymptotic states as tensor products of these normalised single particle states. In order to avoid an unsightly splatter of factors of $2 \pi$, it is convenient to define,

$$
\hat{\delta}^{(n)}(p) \equiv(2 \pi)^{n} \delta^{(n)}(p),
$$

for the $n$-fold Dirac $\delta$ distribution. The scattering matrix $S$ and the transition matrix $T$ are both, of course, dimensionless. We define the amplitudes in four dimensions as usual by

$$
\left\langle p_{1}^{\prime} \cdots p_{m}^{\prime}|T| p_{1} \cdots p_{n}\right\rangle=\mathcal{A}\left(p_{1} \cdots p_{n} \rightarrow p_{1}^{\prime} \cdots p_{m}^{\prime}\right) \hat{\delta}^{(4)}\left(p_{1}+\cdots p_{n}-p_{1}^{\prime}-\cdots-p_{m}^{\prime}\right)
$$

leading to the advertised dimensions for amplitudes.

When restoring powers of $\hbar$, we must distinguish between the momentum $p^{\mu}$ of a particle and its wavenumber, which has dimensions of $[L]^{-1}$. This distinction will be

\footnotetext{
${ }^{1}$ Paraphrasing the late Sidney Coleman, natural units are natural to use because one can always put the units, expressed through $\hbar \mathrm{s}$ and $c \mathrm{~s}$, back through dimensional analysis.
} 
important for us later, so we introduce a notation for the wavenumber $\bar{p}$ associated with a momentum $p$ :

$$
\bar{p} \equiv p / \hbar
$$

In the course of restoring powers of $\hbar$ by dimensional analysis, we first treat the momenta of all particles as genuine momenta. We also treat any mass as a mass rather than the associated Compton wavelength.

When is a point-particle description appropriate? We will consider the scattering of two point-like objects, with momenta $p_{1,2}$, initially separated by a transverse impact parameter $b^{\mu}$. (The impact parameter is transverse in the sense that $p_{i} \cdot b=0$ for $i=1,2$.) At the quantum level, the particles are described by wavefunctions. We will discuss these wavefunctions in more detail in section 4 . We expect the point-particle description to be valid when the separation of the two scattering particles is always very large compared to their (reduced) Compton wavelengths $\ell_{c}^{(i)} \equiv \hbar / m_{i}$, so the point-particle description will be accurate provided that

$$
\sqrt{-b^{2}} \gg \ell_{c}^{(1,2)}
$$

The impact parameter and the Compton wavelengths are not the only scales we must consider, however. The wavefunctions have another intrinsic scale, given by the spread of the wavepackets, $\ell_{w}$. The quantum-mechanical expectation values of observables, as we will discuss, are well-approximated by the corresponding classical ones, when the packet spreads are in the 'Goldilocks' zone, $\ell_{c} \ll \ell_{w} \ll \sqrt{-b^{2}}$.

Let us now imagine restoring the $\hbar$ s in a given amplitude. When $\hbar=1$, the amplitude has dimensions of $[M]^{4-n}$. When $\hbar \neq 1$, the dimensions of the momenta and masses in the amplitude are unchanged. Similarly there is no change to the dimensions of polarisation vectors or tensors or of any Lie-algebraic factors in Yang-Mills theories. However, we must remember that the dimensionless coupling in electrodynamics is $e / \sqrt{\hbar}$. Similarly, in gravity a factor of $1 / \sqrt{\hbar}$ appears as the appropriate coupling with dimensions of inverse mass is $\kappa=\sqrt{32 \pi G / \hbar}$. The algorithm to restore the dimensions of any amplitude in scalar electrodynamics or scalar gravity is thus simple: each factor of a coupling is multiplied by an additional factor of $1 / \sqrt{\hbar}$. For example, an $n$-point, $L$-loop amplitude in scalar QED is proportional to $\hbar^{1-n / 2-L}$.

This conclusion, though well-known, may be surprising in the present context because it seems naively that as $\hbar \rightarrow 0$, higher multiplicities and higher loop orders are more important. As we will see, however, the approach to the classical limits - for observables that make sense classically - effectively forces certain momenta to scale with $\hbar$. These momenta have the classical interpretation of wavenumbers. Examples include the momenta of massless particles, such as photons or gravitons. In putting the factors of $\hbar$ back into the couplings, we have therefore not yet made manifest all of the physically relevant factors of $\hbar$. This provides one motivation for the remainder of our paper: we wish to construct on-shell observables which are both classically and quantum-mechanically sensible. We will then carefully analyse the small- $\hbar$ region to understand how scattering amplitudes encode classical physics. We will see that the appropriate treatment is one where point 
particles have momenta which are fixed as we take $\hbar$ to zero, whereas for massless particles and momentum transfers between massive particles, it is the wavenumber which we should treat as fixed in the limit.

\section{Impulse and radiated momentum in quantum field theory}

We examine scattering events in which two widely separated particles are prepared at $t \rightarrow-\infty$, and then shot at each other with impact parameter $b^{\mu}$. We begin with a discussion of the appropriate incoming state, setting up convenient notation. We then describe the observables of interest.

Our formalism is quite general; for simplicity, we will nonetheless focus on scattering of two stable quanta of different scalar fields with different masses. We will also restrict our attention to scattering processes in which quanta of fields 1 and 2 are both present in the final state. This will happen, for example, if the particles have separately conserved quantum numbers. We also assume that no new quanta of fields 1 and 2 can be produced during the collision, for example because the centre-of-mass energy is too small.

\subsection{The incoming state}

As we prepare the particles in the far past, the appropriate states are incoming states $|\psi\rangle_{\text {in }}$. We describe the incoming particles by wavefunctions $\phi_{i}\left(p_{i}\right)$. The main application we have in mind is to the scattering of point-like classical particles, and so we take our wave functions to have reasonably well-defined positions and momenta. We will discuss the requirements on the wavepackets in considerably more detail in section 4 . In this section we focus on a general discussion of on-shell observables associated with the scattering of localised particles, without specialising to the kinds of wavefunctions which are most appropriate for approaching a classical limit.

The initial state is then,

$$
|\psi\rangle_{\text {in }}=\int \hat{d}^{4} p_{1} \hat{d}^{4} p_{2} \hat{\delta}^{(+)}\left(p_{1}^{2}-m_{1}^{2}\right) \hat{\delta}^{(+)}\left(p_{2}^{2}-m_{2}^{2}\right) \phi_{1}\left(p_{1}\right) \phi_{2}\left(p_{2}\right) e^{i b \cdot p_{1} / \hbar}\left|p_{1} p_{2}\right\rangle_{\text {in }},
$$

where $\hat{d} p$ absorbs a factor of $2 \pi$; more generally $\hat{d}^{n} p$ is defined by

$$
\hat{d}^{n} p \equiv \frac{d^{n} p}{(2 \pi)^{n}} .
$$

We restrict the integration to positive-energy solutions of the delta functions of $p_{i}^{2}-m_{i}^{2}$, as indicated by the $(+)$ superscript in $\hat{\delta}^{(+)}$, as well as absorbing a factor of $2 \pi$ just as for $\hat{\delta}(p)$,

$$
\hat{\delta}^{(+)}\left(p^{2}-m^{2}\right) \equiv 2 \pi \Theta\left(p^{0}\right) \delta\left(p^{2}-m^{2}\right) .
$$

In eq. (3.1) we have translated the wavepacket of particle 1 relative to particle 2 by the impact parameter $b$. In the following, we will often omit the subscript "in", any unlabeled state being understood to be an "in" state. 
We will find it convenient to further abbreviate the notation for on-shell integrals (over Lorentz-invariant phase space),

$$
d \Phi\left(p_{i}\right) \equiv \hat{d}^{4} p_{i} \hat{\delta}^{(+)}\left(p_{i}^{2}-m_{i}^{2}\right) .
$$

We will generally leave the mass implicit, along with the designation of the integration variable as the first summand when the argument is a sum.

As noted in section 2, we follow the standard convention for normalizing states, so that

$$
\left\langle p^{\prime} \mid p\right\rangle=2 E_{p} \hat{\delta}^{(3)}\left(\boldsymbol{p}-\boldsymbol{p}^{\prime}\right) .
$$

As the expression on the right-hand side is the appropriately normalized delta function for the on-shell measure,

$$
\int d \Phi\left(p_{1}^{\prime}\right) 2 E_{p_{1}^{\prime}} \hat{\delta}^{(3)}\left(\boldsymbol{p}_{1}-\boldsymbol{p}_{1}^{\prime}\right) f\left(p_{1}^{\prime}\right)=f\left(p_{1}\right)
$$

for any function $f\left(p_{1}^{\prime}\right)$, we define,

$$
\hat{\delta}_{\Phi}\left(p_{1}-p_{1}^{\prime}\right) \equiv 2 E_{p_{1}^{\prime}} \hat{\delta}^{(3)}\left(\boldsymbol{p}_{1}-\boldsymbol{p}_{1}^{\prime}\right) .
$$

The argument on the left-hand side is understood as a function of four-vectors. This leads to a notationally clearer version of eq. (3.6):

$$
\int d \Phi\left(p_{1}^{\prime}\right) \hat{\delta}_{\Phi}\left(p_{1}-p_{1}^{\prime}\right) f\left(p_{1}^{\prime}\right)=f\left(p_{1}\right)
$$

and of eq. (3.5):

$$
\left\langle p^{\prime} \mid p\right\rangle=\hat{\delta}_{\Phi}\left(p-p^{\prime}\right)
$$

We can also rewrite eq. (3.1),

$$
|\psi\rangle_{\text {in }}=\int d \Phi\left(p_{1}\right) d \Phi\left(p_{2}\right) \phi_{1}\left(p_{1}\right) \phi_{2}\left(p_{2}\right) e^{i b \cdot p_{1} / \hbar}\left|p_{1} p_{2}\right\rangle_{\text {in }} .
$$

Using this simplified notation, the normalisation condition is

$$
\begin{aligned}
1= & \langle\psi \mid \psi\rangle \\
= & \int d \Phi\left(p_{1}\right) d \Phi\left(p_{2}\right) d \Phi\left(p_{1}^{\prime}\right) d \Phi\left(p_{2}^{\prime}\right) e^{i b \cdot\left(p_{1}-p_{1}^{\prime}\right) / \hbar} \\
& \times \phi_{1}\left(p_{1}\right) \phi_{1}^{*}\left(p_{1}^{\prime}\right) \phi_{2}\left(p_{2}\right) \phi_{2}^{*}\left(p_{2}^{\prime}\right) \hat{\delta}_{\Phi}\left(p_{1}-p_{1}^{\prime}\right) \hat{\delta}_{\Phi}\left(p_{2}-p_{2}^{\prime}\right) \\
= & \int d \Phi\left(p_{1}\right) d \Phi\left(p_{2}\right)\left|\phi_{1}\left(p_{1}\right)\right|^{2}\left|\phi_{2}\left(p_{2}\right)\right|^{2} .
\end{aligned}
$$

We can obtain this normalization by requiring both wavefunctions $\phi_{i}$ to be normalized to unity:

$$
\int d \Phi\left(p_{1}\right)\left|\phi_{1}\left(p_{1}\right)\right|^{2}=1
$$




\subsection{The impulse on a particle}

At a gravitational wave observatory, we are of course interested in the gravitational radiation emitted by the source of interest. We will discuss the radiated momentum in section 3.4. Gravitational waves also carry information about the potential experienced by, for example, a black hole binary system. This observation motivates our interest in an on-shell observable related to the potential. We choose to explore the impulse on a particle during a scattering event: at the classical level, this is simply the total change in the momentum of one of the particles - say particle 1 - during the collision.

To define the observable, we place detectors at asymptotically large distances pointing at the collision region. The detectors measure only the momentum of particle 1 . We assume that these detectors cover all possible scattering angles. Let $\mathbb{P}_{i}^{\mu}$ be the momentum operator for particle $i$; the expectation of the first particle's outgoing momentum $p_{\text {out }, 1}^{\mu}$ is then

$$
\begin{aligned}
\left\langle p_{\text {out }, 1}^{\mu}\right\rangle & ={ }_{\text {out }}\left\langle\psi\left|\mathbb{P}_{1}^{\mu}\right| \psi\right\rangle_{\text {out }} \\
& ={ }_{\text {out }}\left\langle\psi\left|\mathbb{P}_{1}^{\mu} U(\infty,-\infty)\right| \psi\right\rangle_{\text {in }} \\
& ={ }_{\text {in }}\left\langle\psi\left|U(\infty,-\infty)^{\dagger} \mathbb{P}_{1}^{\mu} U(\infty,-\infty)\right| \psi\right\rangle_{\text {in }},
\end{aligned}
$$

where $U(\infty,-\infty)$ is the time evolution operator from the far past to the far future. This evolution operator is just the $S$ matrix, so the expectation value is simply,

$$
\left\langle p_{\text {out }, 1}^{\mu}\right\rangle={ }_{\text {in }}\left\langle\psi\left|S^{\dagger} \mathbb{P}_{1}^{\mu} S\right| \psi\right\rangle_{\text {in }} .
$$

We can insert a complete set of states and rewrite the expectation value as,

$$
\left\langle p_{\text {out }, 1}^{\mu}\right\rangle=\sum_{X} \int d \Phi\left(r_{1}\right) d \Phi\left(r_{2}\right) r_{1}^{\mu}\left|\left\langle r_{1} r_{2} X|S| \psi\right\rangle\right|^{2},
$$

where we can think of the inserted states as the final state of a scattering process. In this equation, $X$ refers to any other particles which may be created. The intermediate state containing $X$ also necessarily contains exactly one particle each corresponding to fields 1 and 2 . Their momenta are denoted by $r_{1,2}$ respectively. The sum over $X$ is a sum over all states, including $X$ empty, and includes phase-space integrals for $X$ non-empty. The phasespace integral over the momenta of particles 1 and 2 along with the sum over states $X$ is what gives a complete sum over all states in the Hilbert space. The expression (3.15) hints at the possibility of evaluating the momentum in terms of on-shell scattering amplitudes.

The physically interesting quantity is rather the change of momentum of the particle during the scattering, so we define,

$$
\left\langle\Delta p_{1}^{\mu}\right\rangle=\left\langle\psi\left|S^{\dagger} \mathbb{P}_{1}^{\mu} S\right| \psi\right\rangle-\left\langle\psi\left|\mathbb{P}_{1}^{\mu}\right| \psi\right\rangle .
$$

This impulse is the difference between the expected outgoing and the incoming momenta of particle 1. It is an on-shell observable, defined in both the quantum and the classical theories. Similarly, we can measure the impulse imparted to particle 2. In terms of the momentum operator, $\mathbb{P}_{2}^{\mu}$, of quantum field 2 , this impulse is evidently,

$$
\left\langle\Delta p_{2}^{\mu}\right\rangle=\left\langle\psi\left|S^{\dagger} \mathbb{P}_{2}^{\mu} S\right| \psi\right\rangle-\left\langle\psi\left|\mathbb{P}_{2}^{\mu}\right| \psi\right\rangle .
$$


Returning to the impulse on particle 1 , we proceed by writing the scattering matrix in terms of the transition matrix $T$ via $S=1+i T$, in order to make contact with the usual scattering amplitudes. The no-scattering (unity) part of the $S$ matrix cancels in the impulse, leaving behind only delta functions that identify the final-state momenta with the initial-state ones in the wavefunction or its conjugate. Using unitarity we obtain the result,

$$
\left\langle\Delta p_{1}^{\mu}\right\rangle=\left\langle\psi\left|i\left[\mathbb{P}_{1}^{\mu}, T\right]\right| \psi\right\rangle+\left\langle\psi\left|T^{\dagger}\left[\mathbb{P}_{1}^{\mu}, T\right]\right| \psi\right\rangle
$$

\subsection{Impulse in terms of amplitudes}

Having established a general expression for the impulse, we turn to expressing it in terms of scattering amplitudes. It is convenient to work on the two terms in equation (3.18) separately. For ease of discussion, we define

$$
\begin{aligned}
I_{(1)}^{\mu} & \equiv\left\langle\psi\left|i\left[\mathbb{P}_{1}^{\mu}, T\right]\right| \psi\right\rangle, \\
I_{(2)}^{\mu} & \equiv\left\langle\psi\left|T^{\dagger}\left[\mathbb{P}_{1}^{\mu}, T\right]\right| \psi\right\rangle,
\end{aligned}
$$

so that the impulse is $\left\langle\Delta p_{1}^{\mu}\right\rangle=I_{(1)}^{\mu}+I_{(2)}^{\mu}$. Expanding the wavefunction in the first term, $I_{(1)}^{\mu}$, we find

$$
\begin{aligned}
I_{(1)}^{\mu}= & \int d \Phi\left(p_{1}\right) d \Phi\left(p_{2}\right) d \Phi\left(p_{1}^{\prime}\right) d \Phi\left(p_{2}^{\prime}\right) e^{i b \cdot\left(p_{1}-p_{1}^{\prime}\right) / \hbar} \phi_{1}\left(p_{1}\right) \phi_{1}^{*}\left(p_{1}^{\prime}\right) \phi_{2}\left(p_{2}\right) \phi_{2}^{*}\left(p_{2}^{\prime}\right) \\
& \times i\left(p_{1}^{\prime \mu}-p_{1}^{\mu}\right)\left\langle p_{1}^{\prime} p_{2}^{\prime}|T| p_{1} p_{2}\right\rangle \\
= & \int d \Phi\left(p_{1}\right) d \Phi\left(p_{2}\right) d \Phi\left(p_{1}^{\prime}\right) d \Phi\left(p_{2}^{\prime}\right) e^{i b \cdot\left(p_{1}-p_{1}^{\prime}\right) / \hbar} \phi_{1}\left(p_{1}\right) \phi_{1}^{*}\left(p_{1}^{\prime}\right) \phi_{2}\left(p_{2}\right) \phi_{2}^{*}\left(p_{2}^{\prime}\right) \\
& \times i \int d \Phi\left(r_{1}\right) d \Phi\left(r_{2}\right)\left(r_{1}^{\mu}-p_{1}^{\mu}\right)\left\langle p_{1}^{\prime} p_{2}^{\prime} \mid r_{1} r_{2}\right\rangle\left\langle r_{1} r_{2}|T| p_{1} p_{2}\right\rangle
\end{aligned}
$$

where in the second form line we have re-inserted the final-state momenta $r_{i}$ in order to make manifest the phase independence of the result. We label the states in the incoming wavefunction by $p_{1,2}$, those in the conjugate ones by $p_{1,2}^{\prime}$. Let us now introduce the momentum shifts $q_{i}=p_{i}^{\prime}-p_{i}$, and then change variables in the integration from the $p_{i}^{\prime}$ to the $q_{i}$. In these variables, the matrix element is,

$$
\begin{aligned}
\left\langle p_{1}^{\prime} p_{2}^{\prime}|T| p_{1} p_{2}\right\rangle & =\mathcal{A}\left(p_{1} p_{2} \rightarrow p_{1}^{\prime}, p_{2}^{\prime}\right) \hat{\delta}^{(4)}\left(p_{1}^{\prime}+p_{2}^{\prime}-p_{1}-p_{2}\right) \\
& =\mathcal{A}\left(p_{1} p_{2} \rightarrow p_{1}+q_{1}, p_{2}+q_{2}\right) \hat{\delta}^{(4)}\left(q_{1}+q_{2}\right)
\end{aligned}
$$

yielding

$$
\begin{aligned}
I_{(1)}^{\mu}= & \int d \Phi\left(p_{1}\right) d \Phi\left(p_{2}\right) d \Phi\left(q_{1}+p_{1}\right) d \Phi\left(q_{2}+p_{2}\right) \\
& \times \phi_{1}\left(p_{1}\right) \phi_{1}^{*}\left(p_{1}+q_{1}\right) \phi_{2}\left(p_{2}\right) \phi_{2}^{*}\left(p_{2}+q_{2}\right) \hat{\delta}^{(4)}\left(q_{1}+q_{2}\right) \\
& \times e^{-i b \cdot q_{1} / \hbar} i q_{1}^{\mu} \mathcal{A}\left(p_{1} p_{2} \rightarrow p_{1}+q_{1}, p_{2}+q_{2}\right) .
\end{aligned}
$$

We remind the reader of the shorthand notation introduced earlier for the phase-space measure,

$$
d \Phi\left(q_{1}+p_{1}\right)=\hat{d}^{4} q_{1} \hat{\delta}\left(\left(p_{1}+q_{1}\right)^{2}-m_{1}^{2}\right) \Theta\left(p_{1}^{0}+q_{1}^{0}\right) .
$$


We can perform the integral over $q_{2}$ in eq. (3.22) using the four-fold delta function. Further relabeling $q_{1} \rightarrow q$, we obtain

$$
\begin{aligned}
I_{(1)}^{\mu}= & \int d \Phi\left(p_{1}\right) d \Phi\left(p_{2}\right) \hat{d}^{4} q \hat{\delta}\left(2 p_{1} \cdot q+q^{2}\right) \hat{\delta}\left(2 p_{2} \cdot q-q^{2}\right) \Theta\left(p_{1}^{0}+q^{0}\right) \Theta\left(p_{2}^{0}-q^{0}\right) \\
& \times e^{-i b \cdot q / \hbar} \phi_{1}\left(p_{1}\right) \phi_{1}^{*}\left(p_{1}+q\right) \phi_{2}\left(p_{2}\right) \phi_{2}^{*}\left(p_{2}-q\right) \\
& \times i q^{\mu} \mathcal{A}\left(p_{1} p_{2} \rightarrow p_{1}+q, p_{2}-q\right) .
\end{aligned}
$$

Unusually for a physical observable, this contribution is linear in the amplitude. We emphasize that the incoming and outgoing momenta of this amplitude do not correspond to the initial- and final-state momenta of the scattering process, but rather both correspond to the initial-state momenta, as they appear in the wavefunction and in its conjugate. The momentum $q$ looks like a momentum transfer if we examine the amplitude alone, but for the physical scattering process it represents a difference between the momentum within the wavefunction and that in the conjugate. We will call it a 'momentum mismatch'. As indicated on the first line of eq. (3.20), we should think of this term as an interference of a standard amplitude with an interactionless forward scattering. Diagrammatically, we have learned that

$$
I_{(1)}^{\mu}=\int d \Phi\left(p_{1}\right) d \Phi\left(p_{2}\right) \hat{d}^{4} q \hat{\delta}\left(2 p_{1} \cdot q+q^{2}\right) \hat{\delta}\left(2 p_{2} \cdot q-q^{2}\right) \Theta\left(p_{1}^{0}+q^{0}\right) \Theta\left(p_{2}^{0}-q^{0}\right)
$$

Turning to the second term, $I_{(2)}^{\mu}$, in the impulse, we again introduce a complete set of states labelled by $r_{1}, r_{2}$ and $X$ so that,

$$
\begin{aligned}
I_{(2)}^{\mu} & =\left\langle\psi\left|T^{\dagger}\left[\mathbb{P}_{1}^{\mu}, T\right]\right| \psi\right\rangle \\
& =\sum_{X} \int d \Phi\left(r_{1}\right) d \Phi\left(r_{2}\right)\left\langle\psi\left|T^{\dagger}\right| r_{1} r_{2} X\right\rangle\left\langle r_{1} r_{2} X\left|\left[\mathbb{P}_{1}^{\mu}, T\right]\right| \psi\right\rangle .
\end{aligned}
$$

As above, we can now expand the wavefunctions. We again label the states in the incoming wavefunction by $p_{1,2}$, those in the conjugate ones by $p_{1,2}^{\prime}$,

$$
\begin{aligned}
I_{(2)}^{\mu}= & \sum_{X} \int \prod_{i=1,2} d \Phi\left(r_{i}\right) d \Phi\left(p_{i}\right) d \Phi\left(p_{i}^{\prime}\right) \phi_{i}\left(p_{i}\right) \phi_{i}^{*}\left(p_{i}^{\prime}\right) e^{i b \cdot\left(p_{1}-p_{1}^{\prime}\right) / \hbar}\left(r_{1}^{\mu}-p_{1}^{\mu}\right) \\
& \times \hat{\delta}^{(4)}\left(p_{1}+p_{2}-r_{1}-r_{2}-r_{X}\right) \hat{\delta}^{(4)}\left(p_{1}^{\prime}+p_{2}^{\prime}-r_{1}-r_{2}-r_{X}\right) \\
& \times \mathcal{A}\left(p_{1}, p_{2} \rightarrow r_{1}, r_{2}, r_{X}\right) \mathcal{A}^{*}\left(p_{1}^{\prime}, p_{2}^{\prime} \rightarrow r_{1}, r_{2}, r_{X}\right) .
\end{aligned}
$$

In this expression, $r_{X}$ denotes the total momentum carried by particles in $X$. The second term in the impulse can thus be interpreted as a weighted cut of an amplitude; the lowest order contribution is a weighted two-particle cut of a one-loop amplitude. 
In order to simplify $I_{(2)}^{\mu}$, let us again define the momentum shifts $q_{i}=p_{i}^{\prime}-p_{i}$, and change variables in the integration from the $p_{i}^{\prime}$ to the $q_{i}$, so that,

$$
\begin{aligned}
I_{(2)}^{\mu}= & \sum_{X} \int \prod_{i=1,2} d \Phi\left(r_{i}\right) d \Phi\left(p_{i}\right) d \Phi\left(q_{i}+p_{i}\right) \phi_{i}\left(p_{i}\right) \phi_{i}^{*}\left(p_{i}+q_{i}\right) e^{-i b \cdot q_{1} / \hbar}\left(r_{1}^{\mu}-p_{1}^{\mu}\right) \\
& \times \hat{\delta}^{(4)}\left(p_{1}+p_{2}-r_{1}-r_{2}-r_{X}\right) \hat{\delta}^{(4)}\left(q_{1}+q_{2}\right) \\
& \times \mathcal{A}\left(p_{1}, p_{2} \rightarrow r_{1}, r_{2}, r_{X}\right) \mathcal{A}^{*}\left(p_{1}+q_{1}, p_{2}+q_{2} \rightarrow r_{1}, r_{2}, r_{X}\right) .
\end{aligned}
$$

We can again perform the integral over $q_{2}$ using the four-fold delta function, and relabel $q_{1} \rightarrow q$ to obtain,

$$
\begin{aligned}
I_{(2)}^{\mu}= & \sum_{X} \int \prod_{i=1,2} d \Phi\left(r_{i}\right) d \Phi\left(p_{i}\right) \hat{d}^{4} q \hat{\delta}\left(2 p_{1} \cdot q+q^{2}\right) \hat{\delta}\left(2 p_{2} \cdot q-q^{2}\right) \Theta\left(p_{1}^{0}+q^{0}\right) \Theta\left(p_{2}^{0}-q^{0}\right) \\
& \times \phi_{1}\left(p_{1}\right) \phi_{2}\left(p_{2}\right) \phi_{1}^{*}\left(p_{1}+q\right) \phi_{2}^{*}\left(p_{2}-q\right) e^{-i b \cdot q / \hbar}\left(r_{1}^{\mu}-p_{1}^{\mu}\right) \\
& \times \hat{\delta}^{(4)}\left(p_{1}+p_{2}-r_{1}-r_{2}-r_{X}\right) \\
& \times \mathcal{A}\left(p_{1}, p_{2} \rightarrow r_{1}, r_{2}, r_{X}\right) \mathcal{A}^{*}\left(p_{1}+q, p_{2}-q \rightarrow r_{1}, r_{2}, r_{X}\right) .
\end{aligned}
$$

The momentum $q$ is again a momentum mismatch. The momentum transfers $w_{i} \equiv r_{i}-p_{i}$ will play an important role in analyzing the classical limit, so its convenient to change variables to them from the final-state momenta $r_{i}$,

$$
\begin{aligned}
I_{(2)}^{\mu}= & \sum_{X} \int \prod_{i=1,2} d \Phi\left(p_{i}\right) \hat{d}^{4} w_{i} \hat{d}^{4} q \hat{\delta}\left(2 p_{i} \cdot w_{i}+w_{i}^{2}\right) \Theta\left(p_{i}^{0}+w_{i}^{0}\right) \\
& \times \hat{\delta}\left(2 p_{1} \cdot q+q^{2}\right) \hat{\delta}\left(2 p_{2} \cdot q-q^{2}\right) \Theta\left(p_{1}^{0}+q^{0}\right) \Theta\left(p_{2}^{0}-q^{0}\right) \\
& \times \phi_{1}\left(p_{1}\right) \phi_{2}\left(p_{2}\right) \phi_{1}^{*}\left(p_{1}+q\right) \phi_{2}^{*}\left(p_{2}-q\right) \\
& \times e^{-i b \cdot q / \hbar} w_{1}^{\mu} \hat{\delta}^{(4)}\left(w_{1}+w_{2}+r_{X}\right) \\
& \times \mathcal{A}\left(p_{1}, p_{2} \rightarrow p_{1}+w_{1}, p_{2}+w_{2}, r_{X}\right) \\
& \times \mathcal{A}^{*}\left(p_{1}+q, p_{2}-q \rightarrow p_{1}+w_{1}, p_{2}+w_{2}, r_{X}\right) .
\end{aligned}
$$

Diagrammatically, this second contribution to the impulse is

$$
\begin{aligned}
I_{(2)}^{\mu}= & \sum_{X} \int \prod_{i=1,2} d \Phi\left(p_{i}\right) \hat{d}^{4} w_{i} \hat{d}^{4} q \hat{\delta}\left(2 p_{i} \cdot w_{i}+w_{i}^{2}\right) \Theta\left(p_{i}^{0}+w_{i}^{0}\right) \\
& \times \hat{\delta}\left(2 p_{1} \cdot q+q^{2}\right) \hat{\delta}\left(2 p_{2} \cdot q-q^{2}\right) \Theta\left(p_{1}^{0}+q^{0}\right) \Theta\left(p_{2}^{0}-q^{0}\right) \\
& \times e^{-i b \cdot q / \hbar} w_{1}^{\mu} \hat{\delta}^{(4)}\left(w_{1}+w_{2}+r_{X}\right)
\end{aligned}
$$




\subsection{The momentum radiated during a collision}

A familiar classical observable is the energy radiated by an accelerating particle, for example during a scattering process. More generally we can compute the four-momentum radiated. In quantum mechanics there is no precise prediction for the energy or the momentum radiated by localised particles; we obtain a continuous spectrum if we measure a large number of events. However we can compute the expectation value of the four-momentum radiated during a scattering process. This is a well-defined observable, and as we will see it is on-shell in the sense that it can be expressed in terms of on-shell amplitudes.

To define the observable, let us again surround the collision with detectors which measure outgoing radiation of some type. We may imagine two different contexts: scattering in electrodynamics with radiation of photons, and gravitational scattering with radiation of gravitons. In both cases, we will call the radiated particles 'messengers'. Let $\mathbb{K}^{\mu}$ be the momentum operator for whatever field is radiated; then the expectation of the radiated momentum is

$$
\begin{aligned}
\left\langle k^{\mu}\right\rangle & ={ }_{\text {out }}\left\langle\psi\left|\mathbb{K}^{\mu} U(\infty,-\infty)\right| \psi\right\rangle_{\text {in }} \\
& ={ }_{\text {in }}\left\langle\psi\left|U(\infty,-\infty)^{\dagger} \mathbb{K}^{\mu} U(\infty,-\infty)\right| \psi\right\rangle_{\text {in }},
\end{aligned}
$$

where $U(\infty,-\infty)$ is again the time evolution operator from the far past to the far future - that is, the $S$ matrix. Once again we can anticipate that the radiation will be expressed in terms of amplitudes. Again rewriting $S=1+i T$, the expectation becomes,

$$
R^{\mu} \equiv\left\langle k^{\mu}\right\rangle={ }_{\text {in }}\left\langle\psi\left|S^{\dagger} \mathbb{K}^{\mu} S\right| \psi\right\rangle_{\text {in }}={ }_{\text {in }}\left\langle\psi\left|T^{\dagger} \mathbb{K}^{\mu} T\right| \psi\right\rangle_{\text {in }},
$$

because $\mathbb{K}^{\mu}|\psi\rangle_{\text {in }}=0$ (there are no quanta of radiation in the incoming state).

We can insert a complete set of states $\left|X k r_{1} r_{2}\right\rangle$ containing at least one radiated messenger of momentum $k$, and write the expectation value of the radiated momentum as follows,

$$
\begin{aligned}
R^{\mu} & =\sum_{X} \int d \Phi(k) d \Phi\left(r_{1}\right) d \Phi\left(r_{2}\right)\left\langle\psi\left|T^{\dagger}\right| k r_{1} r_{2} X\right\rangle k_{X}^{\mu}\left\langle k r_{1} r_{2} X|T| \psi\right\rangle \\
& =\sum_{X} \int d \Phi(k) d \Phi\left(r_{1}\right) d \Phi\left(r_{2}\right) k_{X}^{\mu}\left|\left\langle k r_{1} r_{2} X|T| \psi\right\rangle\right|^{2}
\end{aligned}
$$

In this expression, $X$ can again be empty, and $k_{X}^{\mu}$ is the sum of the explicit messenger momentum $k^{\mu}$ and the momenta of any messengers in the state $X$. Notice that we are including explicit phase-space integrals for particles 1 and 2, consistent with our assumption that the number of these particles is conserved during the process. The state $|k\rangle$ describes a radiated messenger; the phase space integral over $k$ implicitly includes a sum over its helicity. 
Expanding the initial state, we find that the expectation value of the radiated momentum is given by,

$$
\begin{aligned}
R^{\mu}= & \sum_{X} \int d \Phi(k) d \Phi\left(r_{1}\right) d \Phi\left(r_{2}\right) k_{X}^{\mu} \mid \int d \Phi\left(p_{1}\right) d \Phi\left(p_{2}\right) e^{i b \cdot p_{1} / \hbar} \phi_{1}\left(p_{1}\right) \phi_{2}\left(p_{2}\right) \\
& \times\left.\mathcal{A}\left(p_{1}, p_{2} \rightarrow r_{1}, r_{2}, k, r_{X}\right) \hat{\delta}^{(4)}\left(p_{1}+p_{2}-r_{1}-r_{2}-k-r_{X}\right)\right|^{2} \\
= & \sum_{X} \int d \Phi(k) \prod_{i=1,2} d \Phi\left(r_{i}\right) d \Phi\left(p_{i}\right) d \Phi\left(p_{i}^{\prime}\right) \phi_{i}\left(p_{i}\right) \phi_{i}^{*}\left(p_{i}^{\prime}\right) k_{X}^{\mu} e^{i b \cdot\left(p_{1}-p_{1}^{\prime}\right) / \hbar} \\
& \times \mathcal{A}\left(p_{1}, p_{2} \rightarrow r_{1}, r_{2}, k, r_{X}\right) \hat{\delta}^{(4)}\left(p_{1}+p_{2}-r_{1}-r_{2}-k-r_{X}\right) \\
& \times \mathcal{A}^{*}\left(p_{1}^{\prime}, p_{2}^{\prime} \rightarrow r_{1}, r_{2}, k, r_{X}\right) \hat{\delta}^{(4)}\left(p_{1}^{\prime}+p_{2}^{\prime}-r_{1}-r_{2}-k-r_{X}\right) .
\end{aligned}
$$

We can again introduce momentum transfers, $q_{i}=p_{i}^{\prime}-p_{i}$, and trade the integrals over $p_{i}^{\prime}$ for integrals over the $q_{i}$. One of the four-fold $\delta$ functions will again become $\hat{\delta}^{(4)}\left(q_{1}+q_{2}\right)$, and we can use it to perform the $q_{2}$ integrations. We again relabel $q_{1} \rightarrow q$. The integration leaves behind a pair of on-shell $\delta$ functions and positive-energy $\Theta$ functions just as in eqs. (3.24) and (3.30),

$$
\begin{aligned}
R^{\mu}= & \sum_{X} \int d \Phi(k) \prod_{i=1,2} d \Phi\left(r_{i}\right) d \Phi\left(p_{i}\right) \hat{d}^{4} q \phi_{1}\left(p_{1}\right) \phi_{2}\left(p_{2}\right) \phi_{1}^{*}\left(p_{1}+q\right) \phi_{2}^{*}\left(p_{2}-q\right) \\
& \times \hat{\delta}\left(2 p_{1} \cdot q+q^{2}\right) \hat{\delta}\left(2 p_{2} \cdot q-q^{2}\right) \Theta\left(p_{1}^{0}+q^{0}\right) \Theta\left(p_{2}{ }^{0}-q^{0}\right) \\
& \times k_{X}^{\mu} e^{-i b \cdot q / \hbar} \hat{\delta}^{(4)}\left(p_{1}+p_{2}-r_{1}-r_{2}-k-r_{X}\right) \\
& \times \mathcal{A}\left(p_{1}, p_{2} \rightarrow r_{1}, r_{2}, k, r_{X}\right) \mathcal{A}^{*}\left(p_{1}+q, p_{2}-q \rightarrow r_{1}, r_{2}, k, r_{X}\right) .
\end{aligned}
$$

We emphasise that this is an all-orders expression: the amplitude $\mathcal{A}\left(p_{1}, p_{2} \rightarrow r_{1}, r_{2}, k, r_{X}\right)$ includes all loop corrections, though of course it can be expanded in perturbation theory. The corresponding real-emission contributions are present in the sum over states $X$. If we truncate the amplitude at a fixed order in perturbation theory, we should similarly truncate the sum over states. Given that the expectation value is expressed in terms of an on-shell amplitude, it is also appropriate to regard this observable as a fully on-shell quantity. As in eqs. (3.24) and (3.30), $q$ represents a momentum mismatch rather than a momentum transfer. Here too, the scattering momentum transfers $w_{i}=r_{i}-p_{i}$ will play an important role in our later discussion of the classical limit, and it is convenient to change variables from the $r_{i}$ to make use of them,

$$
\begin{aligned}
R^{\mu}= & \sum_{X} \int d \Phi(k) \prod_{i=1,2} d \Phi\left(p_{i}\right) \hat{d}^{4} w_{i} \hat{d}^{4} q \hat{\delta}\left(2 p_{i} \cdot w_{i}+w_{i}^{2}\right) \Theta\left(p_{i}^{0}+w_{i}^{0}\right) \\
& \times \hat{\delta}\left(2 p_{1} \cdot q+q^{2}\right) \hat{\delta}\left(2 p_{2} \cdot q-q^{2}\right) \Theta\left(p_{1}^{0}+q^{0}\right) \Theta\left(p_{2}^{0}-q^{0}\right) \\
& \times \phi_{1}\left(p_{1}\right) \phi_{2}\left(p_{2}\right) \phi_{1}^{*}\left(p_{1}+q\right) \phi_{2}^{*}\left(p_{2}-q\right) \\
& \times k_{X}^{\mu} e^{-i b \cdot q / \hbar} \hat{\delta}^{(4)}\left(w_{1}+w_{2}+k+r_{X}\right) \\
& \times \mathcal{A}\left(p_{1}, p_{2} \rightarrow p_{1}+w_{1}, p_{2}+w_{2}, k, r_{X}\right) \\
& \times \mathcal{A}^{*}\left(p_{1}+q, p_{2}-q \rightarrow p_{1}+w_{1}, p_{2}+w_{2}, k, r_{X}\right)
\end{aligned}
$$


It can be useful to represent the observables diagrammatically. Two equivalent expressions for the radiated momentum are helpful:

$$
\begin{aligned}
R^{\mu}= & \sum_{X} \int d \Phi(k) d \Phi\left(r_{1}\right) d \Phi\left(r_{2}\right) k_{X}^{\mu} \\
& \times \mid \int d \Phi\left(p_{1}\right) d \Phi\left(p_{2}\right) e^{i b \cdot p_{1} / \hbar} \hat{\delta}^{(4)}\left(p_{1}+p_{2}-r_{1}-r_{2}-k-r_{X}\right)
\end{aligned}
$$

which is a direct pictorial interpretation of equation (3.35), and

$$
\begin{aligned}
R^{\mu}= & \sum_{X} \int d \Phi(k) \prod_{i=1,2} d \Phi\left(r_{i}\right) d \Phi\left(p_{i}\right) d \Phi\left(p_{i}^{\prime}\right) k_{X}^{\mu} e^{i b \cdot\left(p_{1}-p_{1}^{\prime}\right) / \hbar} \\
& \times \hat{\delta}^{(4)}\left(p_{1}+p_{2}-r_{1}-r_{2}-k-r_{X}\right) \\
& \times \hat{\delta}^{(4)}\left(p_{1}^{\prime}+p_{2}^{\prime}-r_{1}-r_{2}-k-r_{X}\right)
\end{aligned}
$$

which demonstrates that we can think of the expectation value as the weighted cut of a loop amplitude. As $X$ can be empty, the lowest-order contribution arises from the weighted cut of a two-loop amplitude.

\subsection{Conservation of momentum}

The expectation of the radiated momentum is not completely independent of the impulse. In fact the relation between these quantities is physically rich. In the classical electrodynamics of point particles, for example, the impulse is due to a combination of the usual Lorentz force and the ALD radiation reaction (more precisely, the total time integrals of these forces). The Lorentz force exchanges momentum between particles 1 and 2, while the radiation reaction accounts for the irreversible loss of momentum due to radiation. Of course, the ALD force is a notably subtle issue in the classical theory.

In the quantum theory, there can be no question of violating conservation of momentum, so the quantum observables we have defined must already include all the effects which would classically be attributed to both the Lorentz and ALD forces. In particular it must be the case that our definitions respect conservation of momentum. It is easy to demonstrate this formally to all orders using our definitions. Later, in section 5.4, we will indicate how the radiation reaction is included in the impulse more explicitly. 
Our scattering processes involve two incoming particles. Consider, then,

$$
\begin{aligned}
\left\langle\Delta p_{1}^{\mu}\right\rangle+\left\langle\Delta p_{2}^{\mu}\right\rangle & =\left\langle\psi\left|i\left[\mathbb{P}_{1}^{\mu}+\mathbb{P}_{2}^{\mu}, T\right]\right| \psi\right\rangle+\left\langle\psi\left|T^{\dagger}\left[\mathbb{P}_{1}^{\mu}+\mathbb{P}_{2}^{\mu}, T\right]\right| \psi\right\rangle \\
& =\left\langle\psi\left|i\left[\sum_{i} \mathbb{P}_{i}^{\mu}, T\right]\right| \psi\right\rangle+\left\langle\psi\left|T^{\dagger}\left[\mathbb{P}_{1}^{\mu}+\mathbb{P}_{2}^{\mu}, T\right]\right| \psi\right\rangle,
\end{aligned}
$$

where the sum $\sum \mathbb{P}_{i}^{\mu}$ is over all momentum operators in the theory. The second equality above holds because $\mathbb{P}_{i}^{\mu}|\psi\rangle=0$ for $i \neq 1,2$; only quanta of fields 1 and 2 are present in the incoming state. Next, we use the fact that the total momentum is time independent, in other words

$$
\left[\sum \mathbb{P}_{i}^{\mu}, T\right]=0
$$

where the sum extends over all fields. Consequently,

$$
\left\langle\psi\left|i\left[\mathbb{P}_{1}^{\mu}+\mathbb{P}_{2}^{\mu}, T\right]\right| \psi\right\rangle=\left\langle\psi\left|i\left[\sum_{i} \mathbb{P}_{i}^{\mu}, T\right]\right| \psi\right\rangle=0 .
$$

Thus the first term $\left\langle\psi\left|i\left[\mathbb{P}_{1}^{\mu}, T\right]\right| \psi\right\rangle$ in the impulse (3.18) describes only the exchange of momentum between particles 1 and 2 ; in this sense it is associated with the classical Lorentz force (which shares this property) rather than with the classical ALD force (which does not). The second term in the impulse, on the other hand, includes radiation. To make the situation as clear as possible, let us restrict attention to the case where the only other momentum operator is $\mathbb{K}^{\mu}$, the momentum operator for the messenger field. Then we know that $\left[\mathbb{P}_{1}^{\mu}+\mathbb{P}_{2}^{\mu}+\mathbb{K}^{\mu}, T\right]=0$, and conservation of momentum at the level of expectation values is easy to demonstrate:

$$
\left\langle\Delta p_{1}^{\mu}\right\rangle+\left\langle\Delta p_{2}^{\mu}\right\rangle=-\left\langle\psi\left|T^{\dagger}\left[\mathbb{K}^{\mu}, T\right]\right| \psi\right\rangle=-\left\langle\psi\left|T^{\dagger} \mathbb{K}^{\mu} T\right| \psi\right\rangle=-\left\langle k^{\mu}\right\rangle=-R^{\mu},
$$

once again using the fact that there are no messengers in the incoming state.

In the classical theory, radiation reaction is a subleading effect, entering for two-body scattering at order $e^{6}$ in perturbation theory in electrodynamics. This is also the case in the quantum theory. To see why, we again expand the operator product in the second term of eq. (3.18) using a complete set of states:

$$
\left\langle\psi\left|T^{\dagger}\left[\mathbb{P}_{1}^{\mu}, T\right]\right| \psi\right\rangle=\sum_{X} \int d \Phi\left(r_{1}\right) d \Phi\left(r_{2}\right)\left\langle\psi\left|T^{\dagger}\right| r_{1} r_{2} X\right\rangle\left\langle r_{1} r_{2} X\left|\left[\mathbb{P}_{1}^{\mu}, T\right]\right| \psi\right\rangle
$$

The sum over $X$ is over all states, including an implicit integral over their momenta and a sum over any other quantum numbers. The inserted-state momenta of particles 1 and 2 (necessarily present) are labeled by $r_{i}$, and the corresponding integrations over these momenta by $d \Phi\left(r_{i}\right)$. These will ultimately become integrations over the final-state momenta in the scattering. To make the loss of momentum due to radiation explicit at this level, we note that

$$
\left\langle\psi\left|T^{\dagger}\left[\mathbb{P}_{1}^{\mu}+\mathbb{P}_{2}^{\mu}, T\right]\right| \psi\right\rangle=-\sum_{X} \int d \Phi\left(r_{1}\right) d \Phi\left(r_{2}\right)\left\langle\psi\left|T^{\dagger}\right| r_{1} r_{2} X\right\rangle\left\langle r_{1} r_{2} X\left|\mathbb{P}_{X}^{\mu} T\right| \psi\right\rangle,
$$

where $\mathbb{P}_{X}$ is the sum over momentum operators of all quantum fields other than the scalars 1 and 2. The sum over all states $X$ will contain, for example, terms where the state $X$ includes 
messengers of momentum $k^{\mu}$ along with other massless particles. We can further restrict attention to the contributions of the messenger's momentum to $\mathbb{P}_{X}^{\mu}$. This contribution produces a net change of momentum of particle 1 given by

$$
-\sum_{X} \int d \Phi(k) d \Phi\left(r_{1}\right) d \Phi\left(r_{2}\right) k^{\mu}\left\langle\psi\left|T^{\dagger}\right| k r_{1} r_{2} X\right\rangle\left\langle k r_{1} r_{2} X|T| \psi\right\rangle=-\left\langle k^{\mu}\right\rangle
$$

with the help of equation (3.34). Thus we explicitly see the net loss of momentum due to radiating messengers. This quantity is suppressed by factors of $g$ because of the additional state. The lowest order case corresponds to $X=\varnothing$; as there are two quanta in $|\psi\rangle$, we must compute the modulus squared of a five-point tree amplitude. The term is proportional to $g^{6}$, where $g$ is the coupling in the elementary three-point amplitude; as far as the impulse is concerned, it is a next-to-next-to-leading order (NNLO) effect. Other particles in the state $X$, and other contributions to its momentum, describe higher-order effects.

\section{Classical point particles}

The two observables we have discussed - the impulse and the expectation value of the radiated momentum - are designed to be well-defined in both the quantum and the classical theories. As we approach the classical limit, these expectation values should reduce to the classical impulse and the classical radiated momentum. This should ensure that we are able to explore the $\hbar \rightarrow 0$ limit.

We have already discussed in section 2 how to make explicit the factors of $\hbar$ in the observables. We must still discuss the issue of suitable wavefunctions. We must first ensure that the chosen wavefunctions have the desired classical limit. At that point, we could in principle perform the full quantum calculation, using the specific wavefunction we choose, and expand in the $\hbar \rightarrow 0$ limit at the end. However, we also want to choose wavefunctions that allow us to approach the limit as early as possible in the calculation, without relying on their detailed properties. This will lead us to impose stronger constraints on the choice than the mere existence of a suitable classical limit.

\subsection{Wavefunctions}

Heuristically, the wavefunctions for the scattered particles must satisfy two separate conditions. We will take these to be wavepackets, characterized by a smearing or spread in momenta. $^{2}$ That spread should not be too large, so that the interaction with the other particle cannot peer into the details of the wavepacket. At the same time, the details of the wavepacket should not be sensitive to quantum effects.

Let us ground our intuition about scales by first examining nonrelativistic wavefunctions. An example of a minimum-uncertainty wavefunction in momentum space (ignoring normalization) for a particle of mass $m$ growing sharper in the $\hbar \rightarrow 0$ limit has the form,

$$
\exp \left(-\frac{\boldsymbol{p}^{2}}{2 \hbar m \ell_{c} / \ell_{w}^{2}}\right)=\exp \left(-\frac{\boldsymbol{p}^{2}}{2 m^{2} \ell_{c}^{2} / \ell_{w}^{2}}\right)
$$

\footnotetext{
${ }^{2}$ Evaluating positions and uncertainties therein in relativistic field theory is a bit delicate, and we will not consider the question in this article.
} 
where $\ell_{c}$ is the particle's Compton wavelength, and where $\ell_{w}$ is an additional parameter with dimensions of length. We can obtain the conjugate in position space by Fouriertransforming,

$$
\exp \left(-\frac{\left(\boldsymbol{x}-\boldsymbol{x}_{0}\right)^{2}}{2 \ell_{w}^{2}}\right)
$$

The precision with which we know the particle's location is given by $\ell_{w}$, which we could take as an intrinsic measure of the wavefunction's spread.

This suggests that in considering relativistic wavefunctions, we should also take the dimensionless parameter controlling the approach to the classical limit in momentum space to be the square of the ratio of the Compton wavelength $\ell_{c}$ to the intrinsic spread $\ell_{w}$,

$$
\xi \equiv\left(\frac{\ell_{c}}{\ell_{w}}\right)^{2} .
$$

We obtain the classical result by studying the behavior of observables as $\xi \rightarrow 0$. Towards the limit the wavefunctions must be sharply peaked around the classical value for the momenta, $\breve{p}_{i}=m_{i} u_{i}$ with the classical four-velocities $u_{i}$ normalized to $u_{i}^{2}=1$. We can express this requirement through the conditions,

$$
\begin{aligned}
\left\langle p_{i}^{\mu}\right\rangle & =\int d \Phi\left(p_{i}\right) p_{i}^{\mu}\left|\phi\left(p_{i}\right)\right|^{2}=m_{i} \breve{u}_{i}^{\mu} f_{p, i}(\xi), \\
f_{p, i}(\xi) & =1+\mathcal{O}\left(\xi^{\beta^{\prime}}\right), \\
\breve{u}_{i} \cdot u_{i} & =1+\mathcal{O}\left(\xi^{\beta^{\prime \prime}}\right), \\
\sigma^{2}\left(p_{i}\right) / m_{i}^{2} & =\left\langle\left(p_{i}-\left\langle p_{i}\right\rangle\right)^{2}\right\rangle / m_{i}^{2} \\
& =\left(\left\langle p_{i}^{2}\right\rangle-\left\langle p_{i}\right\rangle^{2}\right) / m_{i}^{2}=c_{\Delta} \xi^{\beta},
\end{aligned}
$$

where $c_{\Delta}$ is a constant of order unity, and the $\beta_{\mathrm{S}}$ are simple rational exponents. (The integration measure for $p_{i}$ enforces $\left\langle p_{i}^{2}\right\rangle=m_{i}^{2}$.) For the simplest wavefunctions, $\beta=1$. This spread around the classical value is not necessarily positive, as the difference $p_{i}-\left\langle p_{i}^{\mu}\right\rangle$ may be spacelike, and the expectation of its Lorentz square possibly negative. For that reason, we should resist the usual temptation of taking its square root to obtain a variance.

Because of the phase-space integrals over the initial-state momenta, which enforce the on-shell conditions $p_{i}^{2}=m_{i}^{2}$, the only Lorentz invariant built out of each $p_{i}$ is constant, and so the wavefunction cannot usefully depend on it. This means the wavefunction must depend on at least one four-vector parameter. The simplest wavefunctions will depend on exactly one four-vector, which we can think of as the (classical) four-velocity $u$ of the corresponding particle. It can depend only on the dimensionless combination $p \cdot u / m$ in addition to the parameter $\xi$. The simplest form will be a function of these two in the combination $p \cdot u /(m \xi)$, so that large deviations from $m u$ will be suppressed in a classical quantity. The wavefunction will have additional dependence on $\xi$ in its normalization.

To see the meaning of the constraints more quantitatively, let us examine $I_{(1)}^{\mu}(3.24)$ more closely. It has the form of an amplitude integrated over the on-shell phase space for both of the incoming momenta, subject to additional $\delta$ function constraints - and then weighted by a phase $e^{-i b \cdot q / \hbar}$ dependent on the momentum mismatch $q$, and finally 
integrated over all $q$. As one nears the classical limit, the wavefunction and its conjugate should both represent the particle, that is they should be sharply peaked, and in addition their overlap should be $\mathcal{O}(1)$, up to corrections of $\mathcal{O}(\xi)$. The amplitude will vary slowly on the scale of the wavefunction when one is close to the limit. We will integrate the momentum mismatch $q$ over all possible values, so it is somewhat a matter of taste how we normalize it. Nonetheless, if we take $q_{0}$ to be a 'characteristic' value of $q$, requiring the overlap to be $\mathcal{O}(1)$ is equivalent to requiring that $\phi^{*}(p+q)$ does not differ much from $\phi^{*}(p)$, which in turn requires that the derivative at $p$ is small or that,

$$
\frac{q_{0} \cdot u_{i}}{m \xi} \ll 1
$$

If we scale $q$ by $1 / \hbar$, replacing the momentum by a wavenumber, this constraint takes the following form,

$$
\bar{q}_{0} \cdot u_{i} \ell_{w} \ll \sqrt{\xi} .
$$

We next examine another rapidly varying factor that appears in all our integrands, the delta functions in $q$ arising from the on-shell constraints on the conjugate momenta $p_{i}^{\prime}$. These delta functions, appearing in eqs. (3.24), (3.30), (3.37), take the form,

$$
\hat{\delta}\left(2 p_{i} \cdot q+q^{2}\right)=\frac{1}{\hbar m_{i}} \hat{\delta}\left(2 \bar{q} \cdot u_{i}+\ell_{c} \bar{q}^{2}\right) .
$$

The integration over the initial momenta $p_{i}$ and the initial wavefunctions will smear out these delta functions to sharply peaked functions whose scale is the same order as the original wavefunctions. As $\xi$ gets smaller, this function will turn back into a delta function imposed on the $\bar{q}$ integration. In addition to its dependence on $\xi$, it will depend on two additional dimensionless ratios,

$$
\ell_{c} \sqrt{-\bar{q}^{2}} \quad \text { and } \quad \frac{\bar{q} \cdot u_{i}}{\sqrt{-\bar{q}^{2}}} .
$$

(The characteristic momentum mismatch $q$ is necessarily spacelike.) Let us call $1 / \sqrt{-\bar{q}^{2}}$ a 'scattering length' $\ell_{s}$. In terms of this length, our two dimensionless ratios are,

$$
\frac{\ell_{c}}{\ell_{s}} \quad \text { and } \quad \bar{q} \cdot u_{i} \ell_{s}
$$

The argument of the delta function is polynomial in the two ratios, so we expect them to be constrained to be of order an appropriate power of the spread $\xi$. The direction-averaging implicit in the integration over the $p_{i}$ will lead to a constraint on two positive quantities built out of the ratios, so in general we expect both to be constrained independently. As suggested again by the nonrelativistic limit, the smallest reasonable power of $\xi$ we can imagine emerging as a constraint from the later $\bar{q}$ integration is one-half,

$$
\begin{aligned}
\frac{\ell_{c}}{\ell_{s}} & \lesssim \sqrt{\xi}, \\
\bar{q} \cdot u_{i} \ell_{s} & \lesssim \sqrt{\xi} .
\end{aligned}
$$


If we take a higher power of $\xi$, the constraints would grow stronger. If we had not already scaled out a factor of $\hbar$ from $q$, these constraints would make it natural to do so. Combining the second constraint with eq. (4.6), we obtain the constraint $\ell_{w} \ll \ell_{s}$. The first constraint is weaker, $\ell_{w} \lesssim \ell_{s}$, but on physical grounds as well we should expect the stronger one: only if the wavefunction spread is much smaller than the scattering length can we expect the interaction's probing of the internals of the particle to be negligible.

Combining the stronger constraint with $\xi \ll 1$, we obtain our first version of the 'Goldilocks' inequalities,

$$
\ell_{c} \ll \ell_{w} \ll \ell_{s} .
$$

As we shall see later in the explicit evaluation of $I_{(1)}^{\mu}, \ell_{s} \sim \sqrt{-b^{2}}$; this follows on dimensional grounds along with the observation that the integrals in $I_{(1)}^{\mu}$ lead to no large parameter-free dimensionless numbers. This gives us the second version of the 'Goldilocks' requirement,

$$
\ell_{c} \ll \ell_{w} \ll \sqrt{-b^{2}} .
$$

The combination of eq. (4.6) and the second constraint in eq. (4.10) yields a stronger restriction than the first one in eq. (4.10). We should not expect a similar strengthening of the second restriction; the sharp peaking alone will not force the left-hand side to be much smaller than the right-hand side. This means that we should expect $\bar{q} \cdot u_{i}$ to be smaller than, but still of order, $\sqrt{\xi} / \ell_{s}$. If we compare the two terms in the argument to the delta function (4.7), we see that the second term,

$$
\ell_{c} \bar{q}^{2} \sim \frac{\ell_{c}}{\ell_{s}} \frac{1}{\ell_{s}} \ll \frac{\sqrt{\xi}}{\ell_{s}},
$$

so that $\ell_{c} \bar{q}^{2} \ll \bar{q} \cdot u_{i}$, and the second term should be negligible. There is one caveat to the implied simplification, which we will mention below.

In computing the classical observable, we cannot simply set $\xi=0$. Indeed, we don't even want to fully take the $\xi \rightarrow 0$ limit. Rather, we want to take the leading term in that limit. This term may in fact be proportional to a power of $\xi$. To understand this, we should take note of one additional length scale in the problem, namely the classical radius of the point particle. In electrodynamics, this is $\rho_{c l}=e^{2} /(4 \pi m)$. However,

$$
\rho_{c l}=\frac{\hbar e^{2}}{4 \pi \hbar m}=\alpha \ell_{c}
$$

where $\alpha$ is the usual, dimensionless, electromagnetic coupling. Dimensionless ratios of $\rho_{c l}$ to other length scales will be the expansion parameters in classical observables; but as this relation shows, they too will vanish in the $\xi \rightarrow 0$ limit. There are really three dimensionless parameters we must consider: $\xi ; \ell_{w} / \ell_{s}$; and $\rho_{c l} / \ell_{s}$. We want to retain the full dependence on the latter, while considering only effects independent of the first two.

Under the influence of a perturbatively weak interaction (such as electrodynamics or gravity) below the particle-creation threshold, we expect a wavepacket's shape to be distorted slightly, but not radically changed by the scattering. We would expect the outgoing particles to be characterized by wavepackets similar to those of the incoming particles. 
However, using a wavepacket basis of states for the state sums in section 3 would be cumbersome, inconvenient, and computationally less efficient than the plane-wave states we used. We expect the narrow peaking of the wavefunction to impose constraints on the momentum transfers as they appear in higher-order corrections to the impulse $I_{(2)}^{\mu}$, and in the leading contribution to the radiation reaction $R^{\mu}(3.37)$; but we will need to see this narrowness indirectly, via assessments of the spread as in eq. (4.4) rather than directly through the presence of a wavefunction (or wavefunction mismatch) factors in our observables. We can estimate the spread $\sigma^{2}\left(r_{i}\right)$ in a final-state momentum $r_{i}$ as follows,

$$
\begin{aligned}
\sigma^{2}\left(r_{i}\right) / m_{i}^{2} & =\left\langle\left(r_{i}-\left\langle r_{i}\right\rangle\right)^{2}\right\rangle / m_{i}^{2} \\
& =\left(\left\langle r_{i}^{2}\right\rangle-\left\langle r_{i}\right\rangle^{2}\right) / m_{i}^{2} \\
& =1-\left(\left\langle p_{i}\right\rangle+\left\langle\Delta p_{i}\right\rangle\right)^{2} / m_{i}^{2} \\
& =\sigma^{2}\left(p_{i}\right) / m_{i}^{2}-\left\langle\Delta p_{i}\right\rangle \cdot\left(2\left\langle p_{i}\right\rangle+\left\langle\Delta p_{i}\right\rangle\right) / m_{i}^{2} .
\end{aligned}
$$

So long as $\left\langle\Delta p_{i}\right\rangle / m_{i} \lesssim \sigma^{2}\left(p_{i}\right) / m_{i}^{2}$, the second term will not greatly increase the result, and the spread in the final-state momentum will be of the same order as that in the initial-state momentum. Whether this condition holds depends on the details of the wavefunction. Even if it is violated, so long as $\left\langle\Delta p_{i}\right\rangle / m_{i} \lesssim c_{\Delta}^{\prime} \xi^{\beta^{\prime \prime \prime}}$ with $c_{\Delta}^{\prime}$ a constant of $\mathcal{O}(1)$, then the finalstate momentum will have a narrow spread towards the limit. (It would be broader than the initial-state momentum spread, but that does not affect the applicability of our results.)

The magnitude of $\left\langle\Delta p_{i}\right\rangle$ can be determined perturbatively. The leading-order value comes from $I_{(1)}^{\mu}$, with $I_{(2)}^{\mu}$ and radiative corrections contributing yet-smaller corrections. As we shall see, these computations reveal $\left\langle\Delta p_{i}\right\rangle / m_{i}$ to scale like $\sqrt{\xi}$ and be numerically much smaller. This in turn implies that for perturbative consistency, the 'characteristic' values of momentum transfers $w_{i}$ inside the definition of $R$ must also be very small compared to $m_{i} \sqrt{\xi}$. (This constraint is in fact much weaker than implied by the leading-order value of $\left\langle\Delta p_{i}\right\rangle$.) Just as for $q_{0}$ in eq. (4.5), we should scale these momentum transfers by $1 / \hbar$, replacing them by wavenumbers $\bar{w}_{i}$. The corresponding scattering lengths $\ell_{s}^{\prime}=\sqrt{-w_{1,2}^{2}}$ must again satisfy $\ell_{s}^{\prime} \gg \ell_{w}$. If we now examine the energy-momentum-conserving delta function in eq. (3.37), rewritten in terms of momentum transfers,

$$
\hat{\delta}^{(4)}\left(w_{1}+w_{2}+k+r_{X}\right),
$$

we see that all radiated momenta $k$ and $r_{X}$ must likewise be small compared to $m_{i} \sqrt{\xi}$ : all their energy components must be positive and hence no cancellations are possible inside the delta function. The typical values of these momenta should again by scaled by $1 / \hbar$ and replaced by wavenumbers.

What about loop integrations? As we integrate the loop momentum $\ell$ over all values, it is again a matter of taste how we scale it. If it is the momentum of a (virtual) massless line, however, unitarity considerations suggest that as the natural scaling is to remove a factor of $\hbar$ in real-emission contributions, we should likewise do so for virtual lines. More generally, we should scale those differences of the loop momentum with external legs that correspond to massless particles, and replace them by wavenumbers. Moreover, unitarity 
considerations also suggest that we should choose the loop momentum to be that of a massless line in the loop, if there is one.

In general, we may not be able to approach the $\hbar \rightarrow 0$ limit of each contribution to an observable separately, because they may contain terms which are singular, having too many inverse powers of $\hbar$. We find that such singular terms meet one of two fates: they are multiplied by functions which vanish in the regime of validity of the limit; or they cancel in the sum over all contributions. We cannot yet offer a general argument that such troublesome terms necessarily disappear in one of these two manners. We can treat independently contributions whose singular terms ultimately cancel in the sum, so long as we expand each contribution in a Laurent series in $\hbar$.

Integrand factors that appear uniformly in all contributions - that is, factors which appear directly in a final expression after cancellation of terms singular in the $\hbar \rightarrow 0$ limit - can benefit from applying two simplifications to the integrand: setting $p_{i}$ to $m_{i} u_{i}$, and truncating at the lowest order in $\hbar$ or $\xi$. For other factors, we must be careful to expand in a Laurent series. As mentioned above, inside the on-shell delta functions $\hat{\delta}\left(2 p_{i} \cdot \bar{q} \pm \hbar \bar{q}^{2}\right)$ we can neglect the $\hbar \bar{q}^{2}$ term; this is true so long as the factors multiplying these delta functions are not singular in $\hbar$. If they are indeed nonsingular (after summing over terms), we can safely neglect the second term inside such delta functions, and replace them by $\hat{\delta}\left(2 p_{1} \cdot \bar{q}\right)$. A similar argument allows us to neglect the $\hbar \bar{q}^{0}$ term inside the positive-energy theta functions; the $\bar{q}$ integration then becomes independent of them. Similar arguments, and caveats, apply to the squared momentum-transfer terms $\hbar \bar{w}_{i}^{2}$ appearing inside on-shell delta functions in higher-order contributions, along with the energy components $\bar{w}_{i}^{0}$ appearing inside positive-energy theta functions. They can be neglected so long as the accompanying factors are not singular in $\hbar$. If accompanying factors are singular as $\hbar \rightarrow 0$, then we may need to retain such formally suppressed $\hbar \bar{q}^{2}$ or $\hbar \bar{w}_{i}^{2}$ terms inside delta functions. We will see an example of this in the calculation of the NLO contributions to the impulse in section 5 .

It will be convenient to introduce a notation to allow us to manipulate integrands under the eventual approach to the $\hbar \rightarrow 0$ limit; we will use large angle brackets for the purpose,

$$
\left\langle\left\langle f\left(p_{1}, p_{2}, \ldots\right)\right\rangle\right\rangle \equiv \int d \Phi\left(p_{1}\right) d \Phi\left(p_{2}\right)\left|\phi_{1}\left(p_{1}\right)\right|^{2}\left|\phi_{2}\left(p_{2}\right)\right|^{2} f\left(p_{1}, p_{2}, \ldots\right),
$$

where the integration over both $p_{1}$ and $p_{2}$ is implicit. Within the angle brackets, we have approximated $\phi(p+q) \simeq \phi(p)$, and when evaluating the integrals (implicit in the large angle brackets), we will also set $p_{i} \simeq m_{i} u_{i}$, along with the other simplifications discussed above.

\subsection{An example wavefunction}

It will be helpful to look at the scales that arise in calculations in the context of a specific example for a wavefunction. For this purpose we take a linear exponential,

$$
\phi\left(p_{1}\right)=\mathcal{N} m_{1}^{-1} \exp \left[-\frac{p_{1} \cdot u}{\hbar \ell_{c} / \ell_{w}^{2}}\right]=\mathcal{N} m_{1}^{-1} \exp \left[-\frac{p_{1} \cdot u}{m_{1} \xi}\right]
$$


In this function, $u$ is a dimensionless four-vector; we will ultimately identify it as the four-velocity of particle 1 , and normalize it to $u^{2}=1$. (For the moment, we keep its normalization general.) As discussed in the previous section, $\ell_{w}$ characterizes the 'intrinsic' spread of the wavepacket. In spite of the linearity of the exponent in $p_{1}$, this function gives rise to the Gaussian of eq. (4.1) in the nonrelativistic limit in the rest frame of $u$. (The wavefunction shares some features with relativistic wavefunctions discussed in ref. [117].)

The normalization condition (3.12) requires,

$$
\mathcal{N}=\frac{2 \sqrt{2} \pi}{\xi^{1 / 2} K_{1}^{1 / 2}(2 / \xi)},
$$

where $K_{1}$ is a modified Bessel function of the second kind. For details of this computation and following ones, see appendix B. We can compute the spread of the wavepacket straightforwardly, obtaining

$$
\frac{\left\langle\left(\Delta p_{1}\right)^{2}\right\rangle}{\left\langle p_{1}^{2}\right\rangle}=1-\frac{K_{2}^{2}(2 / \xi)}{K_{1}^{2}(2 / \xi)}
$$

As we approach the classical region, where $\xi \rightarrow 0$, the wavefunction indeed becomes sharply peaked, as

$$
\frac{\left\langle\left(\Delta p_{1}\right)^{2}\right\rangle}{\left\langle p_{1}^{2}\right\rangle} \rightarrow-\frac{3}{2} \xi+\mathcal{O}\left(\xi^{2}\right)
$$

Next, let us consider the implications of the on-shell delta function. Examine a wavefunction integral similar to $I_{(1)}^{\mu}$, but with a simpler integrand,

$$
T_{1}=\int d \Phi\left(p_{1}\right) \phi\left(p_{1}\right) \phi^{*}\left(p_{1}+q\right) \hat{\delta}\left(2 p_{1} \cdot q+q^{2}\right) .
$$

With $\phi$ chosen to be the linear exponential (4.18), this integral simplifies,

$$
T_{1}=\frac{1}{\hbar m_{1}} \eta_{1}\left(\bar{q} ; p_{1}\right) \int d \Phi\left(p_{1}\right) \hat{\delta}\left(2 p_{1} \cdot \bar{q} / m_{1}+\hbar \bar{q}^{2} / m_{1}\right)\left|\phi\left(p_{1}\right)\right|^{2},
$$

where we have also replaced $q \rightarrow \hbar \bar{q}$, and where

$$
\eta_{1}\left(\bar{q} ; p_{1}\right)=\exp \left[-\frac{\hbar \bar{q} \cdot u}{m_{1} \xi}\right] .
$$

The remaining integrations in $T_{1}$ are evaluated in appendix $\mathrm{B}$, yielding

$$
T_{1}=\frac{1}{4 \hbar m_{1} \sqrt{(\bar{q} \cdot u)^{2}-\bar{q}^{2}} K_{1}(2 / \xi)} \exp \left[-\frac{2}{\xi} \frac{\sqrt{(\bar{q} \cdot u)^{2}-\bar{q}^{2}}}{\sqrt{-\bar{q}^{2}}} \sqrt{1-\hbar^{2} \bar{q}^{2} /\left(4 m_{1}^{2}\right)}\right] .
$$

(The wavenumber transfer is necessarily spacelike, so that $-\bar{q}^{2}>0$.)

As we approach the $\hbar, \xi \rightarrow 0$ limit, we may expect this function to be concentrated in a small region in $\bar{q}$. Towards the limit, the dependence on the magnitude is just given by the prefactor. To understand the behavior in the boost and angular degrees of freedom, we may note that

$$
\frac{1}{K_{1}(2 / \xi)} \sim \frac{2}{\sqrt{\pi} \sqrt{\xi}} \exp \left[\frac{2}{\xi}\right]
$$


and that $\hbar \sqrt{\xi}$ is of order $\xi$, so that overall $T_{1}$ has the form,

$$
\frac{1}{\xi} \exp \left[-\frac{f(\bar{q})}{\xi}\right]
$$

which becomes a delta function as $\xi \rightarrow 0$ limit. The more detailed discussion in the appendix shows that it has the form,

$$
\delta(\bar{q} \cdot u)
$$

as anticipated in the previous section.

\subsection{Classical impulse}

We have written the impulse in terms of two terms, $\left\langle\Delta p_{1}^{\mu}\right\rangle=I_{(1)}^{\mu}+I_{(2)}^{\mu}$, and expanded these in terms of wavefunctions in equations (3.24) and (3.30). We will now discuss the classical limit of these terms in detail, applying the rules discussed in section 4.1.

We begin with the first and simplest term in the impulse, $I_{(1)}^{\mu}$, given in eq. (3.24), here recast in the notation of eq. (4.17) in preparation,

$$
\begin{aligned}
I_{(1), \mathrm{cl}}^{\mu}= & i\left\langle\left\langle\int \hat{d}^{4} q \hat{\delta}\left(2 p_{1} \cdot q+q^{2}\right) \hat{\delta}\left(2 p_{2} \cdot q-q^{2}\right) \Theta\left(p_{1}^{0}+q^{0}\right) \Theta\left(p_{2}^{0}-q^{0}\right)\right.\right. \\
& \left.\left.\times e^{-i b \cdot q / \hbar} q^{\mu} \mathcal{A}\left(p_{1} p_{2} \rightarrow p_{1}+q, p_{2}-q\right)\right\rangle\right\rangle .
\end{aligned}
$$

Rescale $q \rightarrow \hbar \bar{q}$; drop the $q^{2}$ inside the on-shell delta functions; and also remove the overall factor of $g^{2}$ and accompanying $\hbar$ s from the amplitude, to obtain the leading-order (LO) contribution to the classical impulse,

$$
\begin{aligned}
\Delta p_{1}^{\mu,(0)} \equiv I_{(1), \mathrm{cl}}^{\mu,(0)}= & i \frac{g^{2}}{4}\left\langle\left\langle\hbar^{2} \int \hat{d}^{4} \bar{q} \hat{\delta}\left(\bar{q} \cdot p_{1}\right) \hat{\delta}\left(\bar{q} \cdot p_{2}\right)\right.\right. \\
& \left.\left.\times e^{-i b \cdot \bar{q}} \bar{q}^{\mu} \overline{\mathcal{A}}^{(0)}\left(p_{1}, p_{2} \rightarrow p_{1}+\hbar \bar{q}, p_{2}-\hbar \bar{q}\right)\right\rangle\right\rangle .
\end{aligned}
$$

We denote by $\overline{\mathcal{A}}^{(L)}$ the reduced $L$-loop amplitude, that is the $L$-loop amplitude with a factor of $g / \sqrt{\hbar}$ removed for every interaction: in the electromagnetic case, this removes a factor of $e / \sqrt{\hbar}$, while in the gravitational case, we would remove a factor of $\kappa / \sqrt{\hbar}$. In general, this rescaled fixed-order amplitude depends only on $\hbar$-free ratios of couplings; in pure electrodynamics or gravitational theory, it is independent of couplings. In pure electrodynamics, it depends on the charges of the scattering particles. While it is free of the powers of $\hbar$ discussed in section 2 , it will in general still scale with an overall power of $\hbar$ thanks to dependence on momentum mismatches or transfers. As we shall see in the next section, additional inverse powers of $\hbar$ emerging from $\overline{\mathcal{A}}$ will cancel the $\hbar^{2}$ prefactor and yield a nonvanishing result.

As a reminder, while this contribution to a physical observable is linear in an amplitude, it arises from an expression involving wavefunctions multiplied by their conjugates. This is reflected in the fact that both the 'incoming' and 'outgoing' momenta in the amplitude 
here are in fact initial-state momenta. Any phase which could be introduced by hand in the initial state would thus cancel out of the observable.

The LO classical impulse is special in that only the first term (3.24) contributes. In general, it is only the sum of the two terms in eq. (3.18) that has a well-defined classical limit. We may write this sum as

$$
I_{\mathrm{cl}}^{\mu}=i\left\langle\left\langle\hbar^{-2} \int \hat{d}^{4} q \hat{\delta}\left(2 p_{1} \cdot q+q^{2}\right) \hat{\delta}\left(2 p_{2} \cdot q-q^{2}\right) \Theta\left(p_{1}^{0}+q^{0}\right) \Theta\left(p_{2}^{0}-q^{0}\right) e^{-i b \cdot q / \hbar} \mathcal{I}^{\mu}\right\rangle\right\rangle,
$$

where the impulse kernel $\mathcal{I}^{\mu}$ is defined as,

$$
\begin{aligned}
\mathcal{I}^{\mu} \equiv & \hbar^{2} q^{\mu} \mathcal{A}\left(p_{1} p_{2} \rightarrow p_{1}+q, p_{2}-q\right) \\
& -i \hbar^{2} \sum_{X} \int \prod_{i=1,2} \hat{d}^{4} w_{i} \hat{\delta}\left(2 p_{i} \cdot w_{i}+w_{i}^{2}\right) \Theta\left(p_{i}^{0}+w_{i}^{0}\right) \\
& \times w_{1}^{\mu} \hat{\delta}^{(4)}\left(w_{1}+w_{2}+r_{X}\right) \\
& \times \mathcal{A}\left(p_{1} p_{2} \rightarrow p_{1}+w_{1}, p_{2}+w_{2}, r_{X}\right) \\
& \times \mathcal{A}^{*}\left(p_{1}+q, p_{2}-q \rightarrow p_{1}+w_{1}, p_{2}+w_{2}, r_{X}\right) .
\end{aligned}
$$

The prefactor in eq. (4.31) and the normalization of $\mathcal{I}^{\mu}$ are chosen so that the latter is $\mathcal{O}\left(\hbar^{0}\right)$ in the classical limit. At leading order, the only contribution comes from the treelevel four-point amplitude in the first term, and after passing to the classical limit, we recover eq. (4.30) as expected. At next-to-leading order (NLO), both terms contribute. The contribution from the first term is from the one-loop amplitude, while that from the second term has $X=\varnothing$, so that both the amplitude and conjugate inside the integral are tree-level four-point amplitudes.

Focus on the NLO contributions, and pass to the classical limit. As discussed in section 4.1 we may neglect the $q^{2}$ terms in the delta functions present in eq. (4.31) so long as any singular terms in the impulse kernel cancel. We then rescale $q \rightarrow \hbar \bar{q}$; and remove an overall factor of $g^{4}$ and accompanying $\hbar$ s from the amplitudes. In addition, we may rescale $w \rightarrow \hbar \bar{w}$. However, since singular terms may be present in the individual summands of the impulse kernel - in general, they will cancel against singular terms emerging from the loop integration in the first term in eq. (4.32) — we are not entitled to drop the $w^{2}$ inside the on-shell delta functions. We obtain,

$$
\Delta p_{1}^{\mu,(1)}=i \frac{g^{4}}{4}\left\langle\left\langle\int \hat{d}^{4} \bar{q} \hat{\delta}\left(p_{1} \cdot \bar{q}\right) \hat{\delta}\left(p_{2} \cdot \bar{q}\right) e^{-i b \cdot \bar{q}} \mathcal{I}_{\mathrm{cl}}^{\mu,(1)}\right\rangle\right\rangle,
$$

where,

$$
\begin{aligned}
\mathcal{I}_{\mathrm{cl}}^{\mu,(1)}= & \hbar \bar{q}^{\mu} \overline{\mathcal{A}}^{(1)}\left(p_{1} p_{2} \rightarrow p_{1}+\hbar \bar{q}, p_{2}-\hbar \bar{q}\right) \\
& -i \hbar^{3} \int \hat{d}^{4} \bar{w} \hat{\delta}\left(2 p_{1} \cdot \bar{w}+\hbar \bar{w}^{2}\right) \hat{\delta}\left(2 p_{2} \cdot \bar{w}-\hbar \bar{w}^{2}\right) \bar{w}^{\mu} \\
& \times \overline{\mathcal{A}}^{(0)}\left(p_{1}, p_{2} \rightarrow p_{1}+\hbar \bar{w}, p_{2}-\hbar \bar{w}\right) \\
& \times \overline{\mathcal{A}}^{(0) *}\left(p_{1}+\hbar \bar{q}, p_{2}-\hbar \bar{q} \rightarrow p_{1}+\hbar \bar{w}, p_{2}-\hbar \bar{w}\right) .
\end{aligned}
$$

Once again, we will see in the next section that additional inverse powers of $\hbar$ will arise from the amplitudes, and will yield a finite and nonvanishing answer in the classical limit. 


\subsection{Classical radiation}

Our starting point for obtaining a prediction for the classical limit of the momentum emitted in radiation is eq. (3.37), which we recast in the notation of eq. (4.17),

$$
\begin{aligned}
R_{\mathrm{cl}}^{\mu}= & \sum_{X}\left\langle\left\langle\int d \Phi(k) \prod_{i=1,2} \hat{d}^{4} w_{i} \hat{d}^{4} q \hat{\delta}\left(2 p_{i} \cdot w_{i}+w_{i}^{2}\right) \Theta\left(p_{i}^{0}+w_{i}^{0}\right)\right.\right. \\
& \times \hat{\delta}\left(2 p_{1} \cdot q+q^{2}\right) \hat{\delta}\left(2 p_{2} \cdot q-q^{2}\right) \Theta\left(p_{1}{ }^{0}+q^{0}\right) \Theta\left(p_{2}{ }^{0}-q^{0}\right) \\
& \times k_{X}^{\mu} e^{-i b \cdot q / \hbar} \hat{\delta}^{(4)}\left(w_{1}+w_{2}+k+r_{X}\right) \\
& \times \mathcal{A}\left(p_{1} p_{2} \rightarrow p_{1}+w_{1}, p_{2}+w_{2}, k, r_{X}\right) \\
& \left.\times \mathcal{A}^{*}\left(p_{1}+q, p_{2}-q \rightarrow p_{1}+w_{1}, p_{2}+w_{2}, k, r_{X}\right) 》\right\rangle .
\end{aligned}
$$

Recall that $k_{X}^{\mu}$ is the sum of the messenger momentum $k^{\mu}$ and the momenta of other messengers in $X$. We will determine the classical limit using precisely the same logic as in the previous subsection. Let us again focus on the leading contribution, with $X=\varnothing$. Once again, rescale $q \rightarrow \hbar \bar{q}$, and drop the $q^{2}$ inside the on-shell delta functions. Here, remove an overall factor of $g^{6}$ and accompanying $\hbar$ s from the amplitude and its conjugate. In addition, rescale the momentum transfers $w \rightarrow \hbar \bar{w}$ and the radiation momenta, $k \rightarrow \hbar k$. At leading order there is no sum, so there will be no hidden cancellations, and we may drop the $w_{i}^{2}$ inside the on-shell delta functions to obtain,

$$
\begin{aligned}
R_{\mathrm{cl}}^{\mu,(0)}= & g^{6}\left\langle\left\langle\hbar^{4} \int d \Phi(\bar{k}) \prod_{i=1,2} \hat{d}^{4} \bar{w}_{i} \hat{d}^{4} \bar{q} \hat{\delta}\left(2 \bar{w}_{i} \cdot p_{i}\right) \hat{\delta}\left(2 \bar{q} \cdot p_{1}\right) \hat{\delta}\left(2 \bar{q} \cdot p_{2}\right)\right.\right. \\
& \times \bar{k}^{\mu} e^{-i b \cdot \bar{q}} \hat{\delta}^{(4)}\left(\bar{w}_{1}+\bar{w}_{2}+\bar{k}\right) \\
& \times \overline{\mathcal{A}}^{(0)}\left(p_{1} p_{2} \rightarrow p_{1}+\hbar \bar{w}_{1}, p_{2}+\hbar \bar{w}_{2}, \hbar \bar{k}\right) \\
& \left.\left.\times \overline{\mathcal{A}}^{(0) *}\left(p_{1}+\hbar \bar{q}, p_{2}-\hbar \bar{q} \rightarrow p_{1}+\hbar \bar{w}_{1}, p_{2}+\hbar \bar{w}_{2}, \hbar \bar{k}\right)\right\rangle\right\rangle .
\end{aligned}
$$

We will make use of this expression below to verify that momentum is conserved as expected.

One disadvantage of this expression for the leading order radiated momentum is that it is no longer in a form of an integral over a perfect square, such as shown in eq. (3.38). Nevertheless we can recast eq. (4.35) in such a form. To do so, perform a change of variable, including in the wavefunctions. To begin, it is helpful to write eq. (4.35) as

$$
\begin{aligned}
R_{\mathrm{cl}}^{\mu}= & \sum_{X} \prod_{i=1,2} \int d \Phi\left(p_{i}\right)\left|\phi_{i}\left(p_{i}\right)\right|^{2} \int d \Phi(k) d \Phi\left(w_{i}+p_{i}\right) d \Phi\left(q_{i}+p_{i}\right) \\
& \times \hat{\delta}^{(4)}\left(w_{1}+w_{2}+k+r_{X}\right) \hat{\delta}^{(4)}\left(q_{1}+q_{2}\right) e^{-i b \cdot q_{1} / \hbar} k_{X}^{\mu} \\
& \times \mathcal{A}\left(p_{1}, p_{2} \rightarrow p_{1}+w_{1}, p_{2}+w_{2}, k, r_{X}\right) \\
& \times \mathcal{A}^{*}\left(p_{1}+q_{1}, p_{2}+q_{2} \rightarrow p_{1}+w_{1}, p_{2}+w_{2}, k, r_{X}\right)
\end{aligned}
$$

We will now re-order the integration and perform a change of variables. Let us define $\tilde{p}_{i}=p_{i}-\tilde{w}_{i}, \tilde{q}_{i}=q_{i}+\tilde{w}_{i}$, and $\tilde{w}_{i}=-w_{i}$, changing variables from $p_{i}$ to $\tilde{p}_{i}$, from $q_{i}$ to $\tilde{q}_{i}$, 
and from $w_{i}$ to $\tilde{w}_{i}$,

$$
\begin{aligned}
R_{\mathrm{cl}}^{\mu}= & \sum_{X} \prod_{i=1,2} \int d \Phi\left(\tilde{p}_{i}\right) d \Phi(k) d \Phi\left(\tilde{w}_{i}+\tilde{p}_{i}\right) d \Phi\left(\tilde{q}_{i}+\tilde{p}_{i}\right)\left|\phi_{i}\left(\tilde{p}_{i}+\tilde{w}_{i}\right)\right|^{2} \\
& \times \hat{\delta}^{(4)}\left(\tilde{w}_{1}+\tilde{w}_{2}-k-r_{X}\right) \hat{\delta}^{(4)}\left(\tilde{q}_{1}+\tilde{q}_{2}-k-r_{X}\right) \\
& \times e^{-i b \cdot\left(\tilde{q}_{1}-\tilde{w}_{1}\right) / \hbar} k_{X}^{\mu} \\
& \times \mathcal{A}\left(\tilde{p}_{1}+\tilde{w}_{1}, \tilde{p}_{2}+\tilde{w}_{2} \rightarrow \tilde{p}_{1}, \tilde{p}_{2}, k, r_{X}\right) \\
& \times \mathcal{A}^{*}\left(\tilde{p}_{1}+\tilde{q}_{1}, \tilde{p}_{2}+\tilde{q}_{2} \rightarrow \tilde{p}_{1}, \tilde{p}_{2}, k, r_{X}\right)
\end{aligned}
$$

As the $\tilde{w}_{i}$ implicitly carry a factor of $\hbar$, just as argued in section 4.1 for the momentum mismatch $q$, we may neglect the shift in the wave functions. Dropping the tildes, and associating the $w_{i}$ integrals with $\mathcal{A}$ and the $q_{i}$ integrals with $\mathcal{A}^{*}$, our expression is revealed as an integral over a perfect square,

$$
\begin{aligned}
R_{\mathrm{cl}}^{\mu}= & \sum_{X} \prod_{i=1,2} \int d \Phi\left(p_{i}\right)\left|\phi_{i}\left(p_{i}\right)\right|^{2} \int d \Phi(k) k_{X}^{\mu} \\
& \times \mid \int d \Phi\left(w_{i}+p_{i}\right) \hat{\delta}^{(4)}\left(w_{1}+w_{2}-k-r_{X}\right) \\
& \times\left. e^{i b \cdot w_{1} / \hbar} \mathcal{A}\left(p_{1}+w_{1}, p_{2}+w_{2} \rightarrow p_{1}, p_{2}, k, r_{X}\right)\right|^{2} \\
= & \sum_{X} \prod_{i=1,2}\left\langle\left\langle\int d \Phi(k) k_{X}^{\mu}\right| \int d \Phi\left(w_{i}+p_{i}\right) \hat{\delta}^{(4)}\left(w_{1}+w_{2}-k-r_{X}\right)\right. \\
& \left.\left.\times\left. e^{i b \cdot w_{1} / \hbar} \mathcal{A}\left(p_{1}+w_{1}, p_{2}+w_{2} \rightarrow p_{1}, p_{2}, k, r_{X}\right)\right|^{2}\right\rangle\right\rangle .
\end{aligned}
$$

The perfect-square structure allows us to define a radiation kernel,

$$
\begin{aligned}
\mathcal{R}\left(k, r_{X}\right) \equiv & \hbar^{3 / 2} \prod_{i=1,2} \int d \Phi\left(p_{i}+w_{i}\right) \hat{\delta}^{(4)}\left(w_{1}+w_{2}-k-r_{X}\right) \\
& \times e^{i b \cdot w_{1} / \hbar} \mathcal{A}\left(p_{1}+w_{1}, p_{2}+w_{2} \rightarrow p_{1}, p_{2}, k, r_{X}\right), \\
= & \hbar^{3 / 2} \prod_{i=1,2} \int \hat{d}^{4} w_{i} \hat{\delta}\left(2 p_{i} \cdot w_{i}+w_{i}^{2}\right) \Theta\left(p_{i}^{0}+w_{i}^{0}\right) \hat{\delta}^{(4)}\left(w_{1}+w_{2}-k-r_{X}\right) \\
& \times e^{i b \cdot w_{1} / \hbar} \mathcal{A}\left(p_{1}+w_{1}, p_{2}+w_{2} \rightarrow p_{1}, p_{2}, k, r_{X}\right)
\end{aligned}
$$

so that

$$
R_{\mathrm{cl}}^{\mu}=\sum_{X} \hbar^{-3}\left\langle\left\langle\int d \Phi(k) k_{X}^{\mu}\left|\mathcal{R}\left(k, r_{X}\right)\right|^{2}\right\rangle\right\rangle .
$$

The prefactor along with the normalization of $\mathcal{R}$ are again chosen so that the classical limit of the radiation kernel will be of $\mathcal{O}\left(\hbar^{0}\right)$. As we will see in section 6.2.1, this expression is related to a classical all-order formula for the radiated momentum.

Let us now focus once more on the leading contribution, with $X=\varnothing$. As usual, rescale $w \rightarrow \hbar \bar{w}$, and as above we may drop the $w^{2}$ inside all of the on-shell delta functions. Again, remove an overall factor of $g^{6}$ and accompanying $\hbar$ s from the amplitude and its 
conjugate. We choose to express the leading-order classical radiated momentum in terms of a leading-order radiation kernel,

$$
\begin{aligned}
\mathcal{R}^{(0)}(\bar{k}) \equiv & \hbar^{2} \prod_{i=1,2} \int \hat{d}^{4} \bar{w}_{i} \hat{\delta}\left(2 p_{i} \cdot \bar{w}_{i}\right) \hat{\delta}^{(4)}\left(\bar{w}_{1}+\bar{w}_{2}-\bar{k}\right) e^{i b \cdot \bar{w}_{1}} \\
& \times \overline{\mathcal{A}}^{(0)}\left(p_{1}+\hbar \bar{w}_{1}, p_{2}+\hbar \bar{w}_{2} \rightarrow p_{1}, p_{2}, \hbar \bar{k}\right),
\end{aligned}
$$

so that the leading-order momentum radiated is simply,

$$
R_{\mathrm{cl}}^{\mu,(0)}=g^{6}\left\langle\left\langle\int d \Phi(\bar{k}) \bar{k}^{\mu}\left|\mathcal{R}^{(0)}(\bar{k})\right|^{2}\right\rangle\right\rangle \text {. }
$$

\subsection{Conservation of momentum}

Conservation of momentum certainly holds to all orders, as we saw in section 3.5. In the classical theory, momentum conservation was however historically a controversial issue. It is thus worth making sure that we have not spoiled this critical physical property in our classical impulse discussion in section 4.3, or in our classical radiation discussion, section 4.4. One might worry, for example, that there is a subtlety with the order of limits.

There is no issue at LO and NLO for the impulse, because

$$
\Delta p_{1}^{\mu,(0)}+\Delta p_{2}^{\mu,(0)}=0, \quad \Delta p_{1}^{\mu,(1)}+\Delta p_{2}^{\mu,(1)}=0 .
$$

These follow straightforwardly from the definitions, eq. (4.30) and eq. (4.33). The essential point is that the amplitudes entering into these orders in the impulse conserve momentum for four particles. At LO, for example, using eq. (4.30) the impulse on particle 2 can be written as

$$
\begin{aligned}
\Delta p_{2}^{\mu,(0)}= & i \frac{g^{2}}{4}\left\langle\left\langle\hbar^{2} \int \hat{d}^{4} \bar{q}_{1} \hat{d}^{4} \bar{q}_{2} \hat{\delta}\left(\bar{q}_{1} \cdot p_{1}\right) \hat{\delta}\left(\bar{q}_{1} \cdot p_{2}\right) \hat{\delta}^{(4)}\left(\bar{q}_{1}+\bar{q}_{2}\right)\right.\right. \\
& \left.\left.\times e^{-i b \cdot \bar{q}_{1}} \bar{q}_{2}^{\mu} \overline{\mathcal{A}}^{(0)}\left(p_{1}, p_{2} \rightarrow p_{1}+\hbar \bar{q}_{1}, p_{2}+\hbar \bar{q}_{2}\right)\right\rangle\right\rangle .
\end{aligned}
$$

In this equation, conservation of momentum at the level of the four point amplitude $\overline{\mathcal{A}}^{(0)}\left(p_{1}, p_{2} \rightarrow p_{1}+\hbar \bar{q}_{1}, p_{2}+\hbar \bar{q}_{2}\right)$ is expressed by the presence of the four-fold delta function $\hat{\delta}^{(4)}\left(\bar{q}_{1}+\bar{q}_{2}\right)$. Using this delta function, we may replace $\bar{q}_{2}^{\mu}$ with $-\bar{q}_{1}^{\mu}$ and then integrate over $\bar{q}_{2}$, once again using the delta function. The result is manifestly $-\Delta p_{1}^{\mu,(0)}$, eq. (4.30). A similar calculation goes through at NLO.

In this sense, the scattering is conservative at LO and at NLO. At NNLO, however, we must take radiative effects into account. This backreaction is entirely described by $I_{(2)}^{\mu}$. As indicated in eq. (3.42), $I_{(1)}^{\mu}$ is always conservative. From our perspective here, this is because it involves only four-point amplitudes. Thus to understand conservation of momentum we need to investigate $I_{(2)}^{\mu}$. The lowest order case in which a five point amplitude can enter $I_{(2)}^{\mu}$ is at NNLO. Let us restrict attention to this lowest order case, taking the additional state $X$ to be a messenger. 
Using precisely the methods of previous subsections, the lowest order term in $I_{(2)}^{\mu}$ involving one messenger is, in the classical regime,

$$
\begin{aligned}
I_{(2), \mathrm{cl}}^{\mu,(\mathrm{rad})}= & g^{6}\left\langle\left\langle\hbar^{4} \int d \Phi(\bar{k}) \prod_{i=1,2} \hat{d}^{4} \bar{w}_{i} \hat{d}^{4} \bar{q}_{1} \hat{d}^{4} \bar{q}_{2} \hat{\delta}\left(2 \bar{w}_{i} \cdot p_{i}\right) \hat{\delta}\left(2 \bar{q}_{1} \cdot p_{1}\right) \hat{\delta}\left(2 \bar{q}_{2} \cdot p_{2}\right)\right.\right. \\
& \times e^{-i b \cdot \bar{q}_{1}} \bar{w}_{1}^{\mu} \hat{\delta}^{(4)}\left(\bar{w}_{1}+\bar{w}_{2}+\bar{k}\right) \hat{\delta}^{(4)}\left(\bar{q}_{1}+\bar{q}_{2}\right) \\
& \times \overline{\mathcal{A}}^{(0)}\left(p_{1}, p_{2} \rightarrow p_{1}+\hbar \bar{w}_{1}, p_{2}+\hbar \bar{w}_{2}, \hbar \bar{k}\right) \\
& \left.\left.\times \overline{\mathcal{A}}^{(0) *}\left(p_{1}+\hbar \bar{q}_{1}, p_{2}+\hbar \bar{q}_{2} \rightarrow p_{1}+\hbar \bar{w}_{1}, p_{2}+\hbar \bar{w}_{2}, \hbar \bar{k}\right)\right\rangle\right\rangle
\end{aligned}
$$

To see that this balances the radiated momentum, we use eq. (4.36). The structure of the expressions are almost identical; conservation of momentum holds because the factor $\bar{k}^{\mu}$ in eq. (4.36) is balanced by $\bar{w}_{1}^{\mu}$ in eq. (4.46) and $\bar{w}_{2}^{\mu}$ in the equivalent expression for particle 2.

Thus conservation of momentum continues to hold in our expressions once we have passed to the classical limit, at least through NNLO. At this order there is non-zero momentum radiated, so momentum conservation is non-trivial from the classical point of view. We will see by explicit calculation in later sections that our classical impulse correctly incorporates the impulse from the ALD force in addition to the Lorentz force.

\section{Examples}

To build confidence in the formalism developed in prior sections, let us use it to calculate some classical point-particle observables explicitly. We will work in the context of scalar electrodynamics, with Lagrangian

$$
\mathcal{L}=-\frac{1}{4} F^{\mu \nu} F_{\mu \nu}+\sum_{i=1,2}\left[\left(D^{\mu} \Phi_{i}\right)^{\dagger} D_{\mu} \Phi_{i}-m_{i}^{2} \Phi_{i}^{\dagger} \Phi_{i}\right] .
$$

We take the charges of the fields $\Phi_{i}$ to be $Q_{i}$, with the electromagnetic coupling the usual $e$.

We will begin by studying the impulse at leading and next-to-leading order. Later, in section 6.1, we will study the same quantity by iterating the classical equations of motion. The fact that this iterative classical approach to the impulse is reminiscent of Feynman diagrams was recently highlighted by Damour [85]. Following our discussion of the impulse, we will discuss the momentum radiated before turning to momentum conservation.

\subsection{Leading-order electromagnetic impulse}

We begin by computing the impulse, $\Delta p_{1}^{\mu,(0)}$, on particle 1 at leading order. At this order, only $I_{(1)}^{\mu}$ contributes, as expressed in eq. (4.30). To evaluate the impulse, we must first compute the $2 \rightarrow 2$ tree-level scattering amplitude. The reduced amplitude $\overline{\mathcal{A}}^{(0)}$ is,

$$
i \overline{\mathcal{A}}^{(0)}\left(p_{1} p_{2} \rightarrow p_{1}+\hbar \bar{q}, p_{2}-\hbar \bar{q}\right)=\sum_{p_{2}-\hbar \bar{q}}^{p_{1}+\hbar \bar{q}}=i Q_{1} Q_{2} \frac{4 p_{1} \cdot p_{2}+\hbar^{2} \bar{q}^{2}}{\hbar^{2} \bar{q}^{2}} .
$$


We can neglect the second term in the numerator, which is subleading in the classical limit.

Substituting this expression into eq. (4.30), we obtain,

$$
\Delta p_{1}^{\mu,(0)}=i e^{2} Q_{1} Q_{2}\left\langle\left\langle\int \hat{d}^{4} \bar{q} \hat{\delta}\left(\bar{q} \cdot p_{1}\right) \hat{\delta}\left(\bar{q} \cdot p_{2}\right) e^{-i b \cdot \bar{q}} \frac{p_{1} \cdot p_{2}}{\bar{q}^{2}} \bar{q}^{\mu}\right\rangle .\right.
$$

As promised, the leading-order expression is independent of $\hbar$. Evaluating the $p_{1,2}$ integrals, in the process applying the simplifications explained in section 4.1, namely replacing $p_{i} \rightarrow$ $m_{i} u_{i}$, we find that,

$$
\Delta p_{1}^{\mu,(0)}=i e^{2} Q_{1} Q_{2} \int \hat{d}^{4} \bar{q} \hat{\delta}\left(\bar{q} \cdot u_{1}\right) \hat{\delta}\left(\bar{q} \cdot u_{2}\right) e^{-i b \cdot \bar{q}} \frac{u_{1} \cdot u_{2}}{\bar{q}^{2}} \bar{q}^{\mu} .
$$

This expression has intriguing similarities to quantities that arise in the high-energy limit of two-body scattering [118-135]. The eikonal approximation used there is known to exponentiate, and it would be interesting to explore this connection further.

It is straightforward to perform the integral over $\bar{q}$ in eq. (5.4) to obtain an explicit expression for the leading order impulse. To do so, we work in the rest frame of particle 1 , so that $u_{1}=(1,0,0,0)$. Without loss of generality we can orientate the spatial coordinates in this frame so that particle 2 is moving along the $z$ axis, with proper velocity $u_{2}=$ $(\gamma, 0,0, \gamma \beta)$. We have introduced the standard Lorentz gamma factor $\gamma=u_{1} \cdot u_{2}$ and the velocity parameter $\beta$ satisfying $\gamma^{2}\left(1-\beta^{2}\right)=1$. In terms of these variables, the impulse is

$$
\begin{aligned}
\Delta p_{1}^{\mu,(0)} & =i e^{2} Q_{1} Q_{2} \int \hat{d}^{4} \bar{q} \hat{\delta}\left(\bar{q}^{0}\right) \hat{\delta}\left(\gamma \bar{q}^{0}-\gamma \beta \bar{q}^{3}\right) e^{-i b \cdot \bar{q}} \frac{\gamma}{\bar{q}^{2}} \bar{q}^{\mu} \\
& =-i \frac{e^{2} Q_{1} Q_{2}}{4 \pi^{2}|\beta|} \int d^{2} \bar{q} e^{i \boldsymbol{b} \cdot \overline{\boldsymbol{q}}_{\perp}} \frac{1}{\overline{\boldsymbol{q}}_{\perp}^{2}} \bar{q}^{\mu},
\end{aligned}
$$

where $\bar{q}^{0}=\bar{q}^{3}=0$ and the non-vanishing components of $\bar{q}^{\mu}$ in the $x y$ plane of our corrdinate system are $\overline{\boldsymbol{q}}_{\perp}$. It remains to perform the two dimensional integral over $\overline{\boldsymbol{q}}_{\perp}$, which is easily done using polar coordinates. Let the magnitude of $\overline{\boldsymbol{q}}_{\perp}$ be $\chi$ and orient the $x$ and $y$ axes so that $\boldsymbol{b} \cdot \overline{\boldsymbol{q}}_{\perp}=|\boldsymbol{b}| \chi \cos \theta$. Then the non-vanishing components of $\bar{q}^{\mu}$ are $\bar{q}^{\mu}=(0, \chi \cos \theta, \chi \sin \theta, 0)$ and the impulse is

$$
\begin{aligned}
\Delta p_{1}^{\mu,(0)} & =-i \frac{e^{2} Q_{1} Q_{2}}{4 \pi^{2}|\beta|} \int_{0}^{\infty} d \chi \chi \int_{-\pi}^{\pi} d \theta e^{i|\boldsymbol{b}| \chi \cos \theta} \frac{1}{\chi^{2}}(0, \chi \cos \theta, \chi \sin \theta, 0) \\
& =-i \frac{e^{2} Q_{1} Q_{2}}{4 \pi^{2}|\beta|} \int_{0}^{\infty} d \chi \int_{-\pi}^{\pi} d \theta e^{i|\boldsymbol{b}| \chi \cos \theta}(0, \cos \theta, \sin \theta, 0) \\
& =\frac{e^{2} Q_{1} Q_{2}}{2 \pi|\beta|} \int_{0}^{\infty} d \chi J_{1}(|\boldsymbol{b}| \chi) \hat{\boldsymbol{b}} \\
& =\frac{e^{2} Q_{1} Q_{2}}{2 \pi|\beta|} \frac{\hat{\boldsymbol{b}}}{|\boldsymbol{b}|}
\end{aligned}
$$

where $\hat{\boldsymbol{b}}$ is the spatial unit vector in the direction of the impact parameter. To restore manifest Lorentz invariance, note that

$$
\frac{1}{|\beta|}=\frac{\gamma}{\sqrt{\gamma^{2}-1}}, \quad \frac{\hat{\boldsymbol{b}}}{|\boldsymbol{b}|}=-\frac{b^{\mu}}{b^{2}} .
$$


(Recall that $b^{\mu}$ is spacelike, so $-b^{2}>0$.) With this input, we may write the impulse as,

$$
\Delta p_{1}^{\mu,(0)}=-\frac{e^{2} Q_{1} Q_{2}}{2 \pi} \frac{\gamma}{\sqrt{\gamma^{2}-1}} \frac{b^{\mu}}{b^{2}} .
$$

In the non-relativistic limit this should match a familiar formula: the expansion of the Rutherford scattering angle $\theta(b)$ as a function of the impact parameter. To keep things simple, we consider Rutherford scattering of a light particle (for example, an electron) off a heavy particle (a nucleus). Taking particle 1 to be the moving light particle, particle 2 is very heavy and we work in its rest frame. Expanding the textbook Rutherford result to order $e^{2}$, we find

$$
\theta(b)=2 \tan ^{-1} \frac{e^{2} Q_{1} Q_{2}}{4 \pi m v^{2} b} \simeq \frac{e^{2} Q_{1} Q_{2}}{2 \pi m v^{2} b},
$$

where $v$ is the non-relativistic velocity of the particle. To recover this simple result from equation (5.4), recall that in the non-relativistic limit $\gamma \simeq 1+v^{2} / 2$. The scattering angle, at this order, is simply $\Delta v / v$. We will make use of this frame in later sections as well.

We note in passing that the second term in the numerator of eq. (5.2) is a quantum correction. It will ultimately be suppressed by $\ell_{c}^{2} / b^{2}$, and in addition would contribute only a contact interaction, as it leads to a $\delta^{(2)}(b)$ term in the impulse.

\subsection{Next-to-leading order impulse}

At the next order in perturbation theory, order $e^{4}$, a well-defined classical impulse is only obtained by combining all terms in the impulse $\left\langle\Delta p_{1}^{\mu}\right\rangle$ of order $e^{4}$. As we discussed in section 4.3 , both $I_{(1)}^{\mu}$ and $I_{(2)}^{\mu}$ contribute. We found in eq. (4.33) that the impulse is a simple integral over an impulse kernel $\mathcal{I}_{\mathrm{cl}}^{\mu,(1)}$, defined in eq. (4.34), which has a well-defined classical limit.

The determination of the impulse kernel at this order requires us to compute the four-point one-loop amplitude along with a cut amplitude, that is an integral over a term quadratic in the tree amplitude. As the one-loop amplitude in electrodynamics is simple, we compute it explicitly using on-shell renormalised perturbation theory in Feynman gauge.

The contributions to the impulse in the quantum theory can be divided into three classes, according to the prefactor in the charges they carry: $C_{1}$, those proportional to $Q_{1}^{3} Q_{2} ; C_{2}$, those to $Q_{1}^{2} Q_{2}^{2}$; and $C_{3}$, those to $Q_{1} Q_{2}^{3}$. The first class can be further subdivided into $C_{1 a}$, terms which would be proportional to $Q_{1}\left(Q_{1}^{2}+n_{s} Q_{3}^{2}\right) Q_{2}$ were we to add $n_{s}$ species of a third scalar with charge $Q_{3}$, and into $C_{1 b}$, terms which would retain the simple $Q_{1}^{3} Q_{2}$ prefactor. Likewise, the last class can be further subdivided into $C_{3 a}$, terms which would be proportional to $Q_{1}\left(Q_{2}^{2}+n_{s} Q_{3}^{2}\right) Q_{2}$, and into $C_{3 b}$, those whose prefactor would remain simply $Q_{1} Q_{2}^{3}$.

Classes $C_{1 a}$ and $C_{3 a}$ consist of photon self-energy corrections along with renormalization counterterms. They appear only in the one-loop corrections to the four-point amplitude, in the first term in the impulse kernel $\mathcal{I}_{\mathrm{cl}}^{\mu,(1)}$. We will discuss them in detail in section 5.2.1 below. As one may suspect ab ipso initio, they give no contribution in the classical limit. Likewise, classes $C_{1 b}$ and $C_{3 b}$ consist of vertex corrections, wavefunction 
renormalization, and their counterterms. We will not discuss them in detail, but they too give no contribution in the classical limit.

This leaves us with contributions of class $C_{2}$; these appear in both terms in the impulse kernel. These contributions to the one-loop amplitude in the first term take the form,

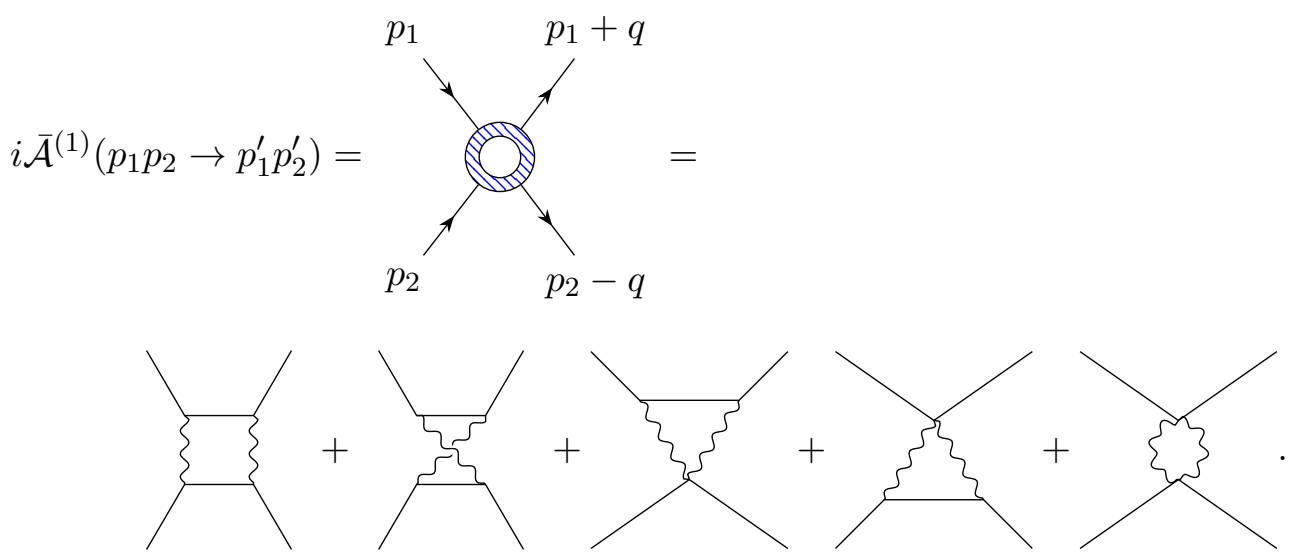

In each contribution, we count powers of $\hbar$ following the rules in section 4.1 , replacing $\ell \rightarrow \hbar \bar{\ell}$ and $q \rightarrow \hbar \bar{q}$. In the double-seagull contribution, we will get four powers from the loop measure, and four inverse powers from the two photon propagators. Overall, we will not get enough inverse powers to compensate the power in front of the integral in eq. (4.34), and so the seagull will die in the classical limit. The remaining diagrams will contribute in the limit, and we discuss them in sects. 5.2.2 and 5.2.3. We discuss the contributions from the second term in the impulse kernel in section 5.2.4, and combine terms in section 5.2.5.

\subsubsection{Purely quantum contributions}

Let us begin with the first and last classes of contributions described in the beginning of this section. These correspond to vertex and photon self-energy terms, including counterterms. Consider, for example, the photon self-energy terms, focussing on internal scalars of mass $m$ and charge $Q_{i}$. Define the self-energy via,

$$
Q_{i}^{2} \Pi\left(q^{2}\right)\left(q^{2} \eta^{\mu \nu}-q^{\mu} q^{\nu}\right) \equiv \underset{q}{\sim} \underset{q}{m}+\underset{q}{m}+\underset{m}{m},
$$

where we have made the projector required by gauge invariance manifest, but have not included factors of the electromagnetic coupling $e$. We have extracted the charges $Q_{i}$ for later convenience. The contribution of the photon self-energy to the reduced four-point amplitude is

$$
\overline{\mathcal{A}}_{\Pi}=Q_{1} Q_{2} Q_{i}^{2} \frac{\left(2 p_{1}+\hbar \bar{q}\right) \cdot\left(2 p_{2}-\hbar \bar{q}\right)}{\hbar^{2} \bar{q}^{2}} \Pi\left(\hbar^{2} \bar{q}^{2}\right) .
$$

The counterterm is adjusted to impose the renormalisation condition that $\Pi(0)=0$, required in order to match the identification of the electromagnetic coupling $e$ with its classical counterpart. As a power series in the dimensionless ratio $q^{2} / m^{2}=\hbar^{2} \bar{q}^{2} / m^{2}$, which is 
of order $\ell_{c}^{2} / b^{2}$,

$$
\Pi\left(q^{2}\right)=\hbar^{2} \Pi^{\prime}(0) \frac{\bar{q}^{2}}{m^{2}}+\mathcal{O}\left(\frac{\ell_{c}^{4}}{b^{4}}\right) .
$$

The renormalization condition is essential in eliminating possible contributions of $\mathcal{O}\left(\hbar^{0}\right)$. One way to see that $\overline{\mathcal{A}}_{\Pi}$ is a purely quantum correction is to follow the powers of $\hbar$. As $\Pi\left(q^{2}\right)$ is of order $\hbar^{2}, \overline{\mathcal{A}}_{\Pi}$ is of order $\hbar^{0}$. This gives a contribution of $\mathcal{O}(\hbar)$ to the impulse kernel (4.34), which in turn gives a contribution of $\mathcal{O}(\hbar)$ to the impulse, as can be seen in eq. (4.33).

Alternatively, one can consider the contribution of these graphs to $\Delta p / p$. Counting each factor of $\bar{q}$ as of order $b$, and using $\Pi\left(q^{2}\right) \sim \ell_{c}^{2} / b^{2}$, it is easy to see that these self-energy graphs yield a contribution to $\Delta p / p$ of order $\alpha^{2} \hbar^{3} /(m b)^{3} \sim\left(\rho_{c l}^{2} / b^{2}\right)\left(\ell_{c} / b\right)$.

The renormalisation of the vertex is similarly a purely quantum effect.

\subsubsection{Triangles}

We turn next to contributions which do survive in the classical limit. Let us first examine the two triangle diagrams in eq. (5.10). They are related by swapping particles 1 and 2 . The first diagram is,

$$
i T_{12}=\sum_{p_{2}-q}^{p_{1}+q}=-2 Q_{1}^{2} Q_{2}^{2} \int \hat{d}^{D} \ell \frac{\left(2 p_{1}+\ell\right) \cdot\left(2 p_{1}+q+\ell\right)}{\ell^{2}(\ell-q)^{2}\left(2 p_{1} \cdot \ell+\ell^{2}+i \epsilon\right)} .
$$

In this integral, we use a dimensional regulator in a standard way $(D=4-2 \epsilon)$ in order to regulate potential divergences. We have retained an explicit $i \epsilon$ in the massive scalar propagator, because it will play an important role below.

To extract the classical contribution of this integral to the amplitude, we recall from section 4.1 that we should set $q=\hbar \bar{q}$ and $\ell=\hbar \bar{\ell}$, and therefore that the components of $q$ and $\ell$ are all small compared to $m$. Consequently, the triangle simplifies to,

$$
T_{12}=\frac{4 i Q_{1}^{2} Q_{2}^{2} m_{1}^{2}}{\hbar} \int \hat{d}^{4} \bar{\ell}_{\overline{\ell^{2}}(\bar{\ell}-\bar{q})^{2}\left(p_{1} \cdot \bar{\ell}+i \epsilon\right)} .
$$

Here, we have taken the limit $D \rightarrow 4$, as the integral is now free of divergences. Notice that we have exposed one additional inverse power of $\hbar$. Comparing to the definition of $\mathcal{I}_{\mathrm{cl}}^{\mu,(1)}$ in eq. (4.34), we see that this inverse power of $\hbar$ will cancel against the explicit factor of $\hbar$ in $I_{(1), \mathrm{cl}}^{\mu,(1)}$, signaling a classical contribution to the impulse.

At this point we employ a simple trick which simplifies the loop integral appearing in eq. (5.15), and which will be of great help in simplifying the more complicated box topologies below. The on-shell condition for the outgoing particle 1 requires that $p_{1} \cdot \bar{q}=$ $-\hbar \bar{q}^{2} / 2$, so replace $\bar{\ell} \rightarrow \bar{\ell}^{\prime}=\bar{q}-\bar{\ell}$ in $T_{12}$ :

$$
\begin{aligned}
T_{12} & =-\frac{4 i Q_{1}^{2} Q_{2}^{2} m_{1}^{2}}{\hbar} \int \hat{d}^{4} \bar{\ell}^{\prime} \frac{1}{\bar{\ell}^{\prime 2}\left(\bar{\ell}^{\prime}-\bar{q}\right)^{2}\left(p_{1} \cdot \bar{\ell}^{\prime}+\hbar \bar{q}^{2}-i \epsilon\right)} \\
& =-\frac{4 i Q_{1}^{2} Q_{2}^{2} m_{1}^{2}}{\hbar} \int \hat{d}^{4} \bar{\ell}^{\prime} \frac{1}{\bar{\ell}^{\prime 2}\left(\bar{\ell}^{\prime}-\bar{q}\right)^{2}\left(p_{1} \cdot \bar{\ell}^{\prime}-i \epsilon\right)}+\mathcal{O}\left(\hbar^{0}\right),
\end{aligned}
$$


Because of the linear power of $\hbar$ appearing in eq. (4.34), the second term is in fact a quantum correction. We therefore neglect it, and write,

$$
T_{12}=-\frac{4 i Q_{1}^{2} Q_{2}^{2} m_{1}^{2}}{\hbar} \int \hat{d}^{4} \bar{\ell}_{\bar{\ell}^{2}(\bar{\ell}-\bar{q})^{2}\left(p_{1} \cdot \bar{\ell}-i \epsilon\right)},
$$

where we have dropped the prime on the loop momentum: $\ell^{\prime} \rightarrow \ell$. Comparing with our previous expression, eq. (5.15), for the triangle, the net result of these replacements has simply been to introduce an overall sign while, crucially, also switching the sign of the $i \epsilon$ term. Symmetrising over the two expressions for $T_{12}$, we learn that

$$
T_{12}=\frac{2 Q_{1}^{2} Q_{2}^{2} m_{1}^{2}}{\hbar} \int \hat{d}^{4} \bar{\ell} \frac{\hat{\delta}\left(p_{1} \cdot \bar{\ell}\right)}{\bar{\ell}^{2}(\bar{\ell}-\bar{q})^{2}}
$$

using the identity

$$
\frac{1}{x-i \epsilon}-\frac{1}{x+i \epsilon}=i \hat{\delta}(x)
$$

The second triangle contributing to the amplitude, $T_{21}$, can be obtained from $T_{12}$ simply by interchanging the labels 1 and 2 :

$$
T_{21}=\frac{2 Q_{1}^{2} Q_{2}^{2} m_{2}^{2}}{\hbar} \int \hat{d}^{4} \bar{\ell} \frac{\hat{\delta}\left(p_{2} \cdot \bar{\ell}\right)}{\bar{\ell}^{2}(\bar{\ell}-\bar{q})^{2}} .
$$

These triangles contribute to the impulse kernel via

$$
\begin{aligned}
\left.\mathcal{I}_{\mathrm{cl}}^{\mu,(1)}\right|_{\text {triangle }} & =\hbar \bar{q}^{\mu}\left(T_{12}+T_{21}\right) \\
& =2 Q_{1}^{2} Q_{2}^{2} \bar{q}^{\mu} \int \frac{\hat{d}^{4} \bar{\ell}}{\bar{\ell}^{2}(\bar{\ell}-\bar{q})^{2}}\left(m_{1}^{2} \hat{\delta}\left(p_{1} \cdot \bar{\ell}\right)+m_{2}^{2} \hat{\delta}\left(p_{2} \cdot \bar{\ell}\right)\right) .
\end{aligned}
$$

Recall that we must integrate over the wavefunctions in order to obtain the classical impulse from the impulse kernel. As we have discussed in section 4.1, because the inverse power of $\hbar$ here is canceled by the linear power present explicitly in eq. (4.33), we may evaluate the wavefunction integrals by replacing the $p_{i}$ by their classical values $m_{i} u_{i}$. The result for the contribution to the kernel is,

$$
\mathcal{I}_{1}^{\mu} \equiv 2 Q_{1}^{2} Q_{2}^{2} \bar{q}^{\mu} \int \hat{d}^{4} \bar{\ell} \frac{1}{\bar{\ell}^{2}(\bar{\ell}-\bar{q})^{2}}\left(m_{1} \hat{\delta}\left(u_{1} \cdot \bar{\ell}\right)+m_{2} \hat{\delta}\left(u_{2} \cdot \bar{\ell}\right)\right) .
$$

One must still integrate this expression over $\bar{q}$ as in eq. (4.33) to obtain the contribution to the impulse.

\subsubsection{Boxes}

The one-loop amplitude also includes boxes, crossed boxes, and the NLO contribution to the impulse includes as well a term quadratic in the tree amplitude which we can think of as the cut of a one-loop box. Because of the power of $\hbar$ in front of the first term in eq. (4.34), we need to extract the contributions of all of these quantities at order $1 / \hbar$. However, as we will see, each individual diagram also contains singular terms of order $1 / \hbar^{2}$. We might fear that 
these terms pose an obstruction to the very existence of a classical limit of the observable in which we are interested. As we will see, this fear is misplaced, as these singular terms cancel completely, leaving a well-defined classical result. It is straightforward to evaluate the individual contributions, but making the cancellation explicit requires some care. We begin with the box:

$$
\begin{aligned}
& i B=\ell \uparrow \sum_{p_{2}-q}=Q_{1}^{2} Q_{2}^{2} \int \hat{d}^{D} \ell \frac{\left(2 p_{1}+\ell\right) \cdot\left(2 p_{2}-\ell\right)\left(2 p_{1}+q+\ell\right) \cdot\left(2 p_{2}-q-\ell\right)}{\ell^{2}(\ell-q)^{2}\left(2 p_{1} \cdot \ell+\ell^{2}+i \epsilon\right)\left(-2 p_{2} \cdot \ell+\ell^{2}+i \epsilon\right)}= \\
& \frac{Q_{1}^{2} Q_{2}^{2}}{\hbar^{2+2 \epsilon}} \int \hat{d}^{D} \bar{\ell} \frac{\left[4 p_{1} \cdot p_{2}-2 \hbar\left(p_{1}-p_{2}\right) \cdot \bar{\ell}-\hbar^{2} \bar{\ell}^{2}\right]\left[4 p_{1} \cdot p_{2}-2 \hbar\left(p_{1}-p_{2}\right) \cdot(\bar{\ell}+\bar{q})-\hbar^{2}(\bar{\ell}+\bar{q})^{2}\right]}{\bar{\ell}^{2}(\bar{\ell}-\bar{q})^{2}\left(2 p_{1} \cdot \bar{\ell}+\hbar \bar{\ell}^{2}+i \epsilon\right)\left(-2 p_{2} \cdot \bar{\ell}+\hbar \bar{\ell}^{2}+i \epsilon\right)},
\end{aligned}
$$

where as usual, we have set $q=\hbar \bar{q}, \ell=\hbar \bar{\ell}$. We get four powers of $\hbar$ from changing variables in the measure, but six inverse powers from the propagators. ${ }^{3}$ We thus encounter an apparently singular $1 / \hbar^{2}$ leading behaviour. We must extract both this singular, $\mathcal{O}\left(1 / \hbar^{2}\right)$, term as well as the terms contributing in the classical limit, which here are $\mathcal{O}(1 / \hbar)$. Consequently, we must take care to remember that the on-shell delta functions enforce $\bar{q} \cdot p_{1}=-\hbar \bar{q}^{2} / 2$ and $\bar{q} \cdot p_{2}=\hbar \bar{q}^{2} / 2$.

Performing a Laurent expansion in $\hbar$, truncating after order $1 / \hbar$, and separating different orders in $\hbar$, we find that the box is given by,

$$
\begin{aligned}
& B=B_{-1}+B_{0}, \\
& B_{-1}=\frac{4 i Q_{1}^{2} Q_{2}^{2}}{\hbar^{2+2 \epsilon}}\left(p_{1} \cdot p_{2}\right)^{2} \int \frac{\hat{d}^{D} \bar{\ell}}{\bar{\ell}^{2}(\bar{\ell}-\bar{q})^{2}\left(p_{1} \cdot \bar{\ell}+i \epsilon\right)\left(p_{2} \cdot \bar{\ell}-i \epsilon\right)}, \\
& B_{0}=-\frac{2 i Q_{1}^{2} Q_{2}^{2}}{\hbar^{1+2 \epsilon}} p_{1} \cdot p_{2} \int \frac{\hat{d}^{D} \bar{\ell}}{\bar{\ell}^{2}(\bar{\ell}-\bar{q})^{2}\left(p_{1} \cdot \bar{\ell}+i \epsilon\right)\left(p_{2} \cdot \bar{\ell}-i \epsilon\right)} \\
& \times\left[2\left(p_{1}-p_{2}\right) \cdot \bar{\ell}+\frac{\left(p_{1} \cdot p_{2}\right) \bar{\ell}^{2}}{\left(p_{1} \cdot \bar{\ell}+i \epsilon\right)}-\frac{\left(p_{1} \cdot p_{2}\right) \bar{\ell}^{2}}{\left(p_{2} \cdot \bar{\ell}-i \epsilon\right)}\right] .
\end{aligned}
$$

Note that pulling out a sign from one of the denominators has given the appearance of flipping the sign of one of the denominator $i \epsilon$ terms. We must also bear in mind that the integral in $B_{-1}$ is itself not $\hbar$-independent, so that we will later need to expand it as well.

\footnotetext{
${ }^{3}$ We omit fractional powers of $\hbar$ in this counting as they will disappear when we take $D \rightarrow 4$.
} 
Similarly, the crossed box is

$$
\begin{aligned}
& =Q_{1}^{2} Q_{2}^{2} \int \hat{d}^{D} \ell \frac{\left(2 p_{1}+\ell\right) \cdot\left(2 p_{2}-2 q+\ell\right)\left(2 p_{1}+q+\ell\right) \cdot\left(2 p_{2}-q+\ell\right)}{\ell^{2}(\ell-q)^{2}\left(2 p_{1} \cdot \ell+\ell^{2}+i \epsilon\right)\left(2 p_{2} \cdot(\ell-q)+(\ell-q)^{2}+i \epsilon\right)} \\
& =\frac{Q_{1}^{2} Q_{2}^{2}}{\hbar^{2}+2 \epsilon} \int \hat{d}^{D} \bar{\ell} \frac{\left(2 p_{1}+\hbar \bar{\ell}\right) \cdot\left(2 p_{2}-2 \hbar \bar{q}+\hbar \bar{\ell}\right)\left(2 p_{1}+\hbar \bar{q}+\hbar \bar{\ell}\right) \cdot\left(2 p_{2}-\hbar \bar{q}+\hbar \bar{\ell}\right)}{\bar{\ell}^{2}(\bar{\ell}-\bar{q})^{2}\left(2 p_{1} \cdot \bar{\ell}+\hbar \bar{\ell}^{2}+i \epsilon\right)\left(2 p_{2} \cdot(\bar{\ell}-\bar{q})+\hbar(\bar{\ell}-\bar{q})^{2}+i \epsilon\right)} .
\end{aligned}
$$

Using the on-shell conditions to simplify $p_{i} \cdot \bar{q}$ terms in the denominator and numerator, and once again expanding in powers of $\hbar$, truncating after order $1 / \hbar$, and separating different orders in $\hbar$, we find,

$$
\begin{aligned}
C= & C_{-1}+C_{0}, \\
C_{-1}= & -\frac{4 i Q_{1}^{2} Q_{2}^{2}}{\hbar^{2+2 \epsilon}}\left(p_{1} \cdot p_{2}\right)^{2} \int \frac{\hat{d}^{D} \bar{\ell}}{\bar{\ell}^{2}(\bar{\ell}-\bar{q})^{2}} \frac{1}{\left(p_{1} \cdot \bar{\ell}+i \epsilon\right)\left(p_{2} \cdot \bar{\ell}+i \epsilon\right)} \\
C_{0}= & -\frac{2 i Q_{1}^{2} Q_{2}^{2}}{\hbar^{1+2 \epsilon}} p_{1} \cdot p_{2} \int \frac{\hat{d}^{D} \bar{\ell}}{\bar{\ell}^{2}(\bar{\ell}-\bar{q})^{2}\left(p_{1} \cdot \bar{\ell}+i \epsilon\right)\left(p_{2} \cdot \bar{\ell}+i \epsilon\right)} \\
& \times\left[2\left(p_{1}+p_{2}\right) \cdot \bar{\ell}-\frac{\left(p_{1} \cdot p_{2}\right) \bar{\ell}^{2}}{\left(p_{1} \cdot \bar{\ell}+i \epsilon\right)}-\frac{\left(p_{1} \cdot p_{2}\right)\left[(\bar{\ell}-\bar{q})^{2}-\bar{q}^{2}\right]}{\left(p_{2} \cdot \bar{\ell}+i \epsilon\right)}\right] .
\end{aligned}
$$

Comparing the expressions for the $\mathcal{O}\left(1 / \hbar^{2}\right)$ terms in the box and the crossed box, $B_{-1}$ and $C_{-1}$ respectively, we see that there is only a partial cancellation of the singular, $\mathcal{O}\left(1 / \hbar^{2}\right)$, term in the reduced amplitude $\overline{\mathcal{A}}^{(1)}$. The impulse kernel, eq. (4.34), does contain another term, which is quadratic in the tree-level reduced amplitude $\overline{\mathcal{A}}^{(0)}$. We will see below that taking this additional contribution into account leads to a complete cancellation of the singular term; but the classical limit does not exist for each of these terms separately.

\subsubsection{Cut box}

In order to see the cancellation of the singular term we must incorporate the term in the impulse kernel which is quadratic in tree amplitudes. This contribution can be viewed as proportional to the cut of the one-loop box, weighted by the loop momentum $\hbar \bar{w}^{\mu}$ :

$$
\left.B^{\mu}=-i \hbar^{2} \int \hat{d}^{4} \bar{w} \bar{w}^{\mu} \hat{\delta}\left(2 p_{1} \cdot \bar{w}+\hbar \bar{w}^{2}\right) \hat{\delta}\left(2 p_{2} \cdot \bar{w}-\hbar \bar{w}^{2}\right) \times \hbar \bar{w}^{\uparrow}\right\}_{p_{2}-\hbar \bar{q}},
$$

where an additional factor of $\hbar$ in the second term of eq. (4.34) will be multiplied into eq. (5.32) below, as it parallels the factor in the first term of eq. (4.34). Evaluating the 
Feynman diagrams, we obtain,

$$
\begin{aligned}
B^{\mu}= & -i \frac{Q_{1}^{2} Q_{2}^{2}}{\hbar^{2}} \int \hat{d}^{4} \bar{w} \hat{\delta}\left(2 p_{1} \cdot \bar{w}+\hbar \bar{w}^{2}\right) \hat{\delta}\left(2 p_{2} \cdot \bar{w}-\hbar \bar{w}^{2}\right) \frac{\bar{w}^{\mu}}{\bar{w}^{2}(\bar{w}-\bar{q})^{2}} \\
& \times\left(2 p_{1}+\hbar \bar{w}\right) \cdot\left(2 p_{2}-\bar{w} \hbar\right)\left(2 p_{1}+\hbar \bar{q}+\hbar \bar{w}\right) \cdot\left(2 p_{2}-\hbar \bar{q}-\hbar \bar{w}\right) .
\end{aligned}
$$

As in the previous subsection, expand in $\hbar$, and truncate after order $1 / \hbar$, so that,

$$
\begin{aligned}
B^{\mu} & =B_{-1}^{\mu}+B_{0}^{\mu} \\
B_{-1}^{\mu} & =-\frac{4 i Q_{1}^{2} Q_{2}^{2}}{\hbar^{2}}\left(p_{1} \cdot p_{2}\right)^{2} \int \frac{\hat{d}^{4} \bar{\ell} \bar{\ell}^{\mu}}{\bar{\ell}^{2}(\bar{\ell}-\bar{q})^{2}} \hat{\delta}\left(p_{1} \cdot \bar{\ell}\right) \hat{\delta}\left(p_{2} \cdot \bar{\ell}\right) \\
B_{0}^{\mu} & =-\frac{2 i Q_{1}^{2} Q_{2}^{2}}{\hbar}\left(p_{1} \cdot p_{2}\right)^{2} \int \frac{\hat{d}^{4} \bar{\ell} \bar{\ell}^{\mu}}{\bar{\ell}^{2}(\bar{\ell}-\bar{q})^{2}} \bar{\ell}^{2}\left(\hat{\delta}^{\prime}\left(p_{1} \cdot \bar{\ell}\right) \hat{\delta}\left(p_{2} \cdot \bar{\ell}\right)-\hat{\delta}\left(p_{1} \cdot \bar{\ell}\right) \hat{\delta}^{\prime}\left(p_{2} \cdot \bar{\ell}\right)\right) .
\end{aligned}
$$

We have relabeled $\bar{w} \rightarrow \bar{\ell}$ in order to line up terms more transparently with corresponding ones in the box and crossed box contributions.

\subsubsection{Combining contributions}

We are now in a position to assemble the elements computed in the three previous subsections in order to obtain the NLO contributions to the impulse kernel $\mathcal{I}_{\mathrm{cl}}^{\mu,(1)}$, and thence the NLO contributions to the impulse using eq. (4.33). Let us begin by examining the singular terms. We must combine the terms from the box, crossed box, and cut box.

We can simplify the cut-box contribution $B_{-1}^{\mu}$ by exploiting the linear change of variable $\bar{\ell}^{\prime}=\bar{q}-\bar{\ell}$

$$
\begin{aligned}
B_{-1}^{\mu} & =-\frac{4 i Q_{1}^{2} Q_{2}^{2}}{\hbar^{2}}\left(p_{1} \cdot p_{2}\right)^{2} \int \hat{d}^{4} \bar{\ell}^{\prime} \frac{\left(\bar{q}^{\mu}-\bar{\ell}^{\prime \mu}\right)}{\bar{\ell}^{\prime 2}\left(\bar{\ell}^{\prime}-\bar{q}\right)^{2}} \hat{\delta}\left(p_{1} \cdot \bar{\ell}^{\prime}-p_{1} \cdot \bar{q}\right) \hat{\delta}\left(p_{2} \cdot \bar{\ell}^{\prime}-p_{2} \cdot \bar{q}\right) \\
& =-\frac{4 i Q_{1}^{2} Q_{2}^{2}}{\hbar^{2}}\left(p_{1} \cdot p_{2}\right)^{2} \int \hat{d}^{4} \bar{\ell}^{\prime} \frac{\left(\bar{q}^{\mu}-\bar{\ell}^{\prime \mu}\right)}{\bar{\ell}^{\prime 2}\left(\bar{\ell}^{\prime}-\bar{q}\right)^{2}} \hat{\delta}\left(p_{1} \cdot \bar{\ell}^{\prime}+\hbar \bar{q}^{2} / 2\right) \hat{\delta}\left(p_{2} \cdot \bar{\ell}^{\prime}-\hbar \bar{q}^{2} / 2\right) \\
& =-\frac{2 i Q_{1}^{2} Q_{2}^{2}}{\hbar^{2}}\left(p_{1} \cdot p_{2}\right)^{2} \bar{q}^{\mu} \int \hat{d}^{4} \overline{\bar{\ell}} \frac{\hat{\delta}\left(p_{1} \cdot \bar{\ell}\right) \hat{\delta}\left(p_{2} \cdot \bar{\ell}\right)}{\bar{\ell}^{2}(\bar{\ell}-\bar{q})^{2}}+\mathcal{O}(1 / \hbar),
\end{aligned}
$$

where we have used the on-shell conditions to replace $p_{1} \cdot \bar{q} \rightarrow-\hbar \bar{q}^{2} / 2$ and $p_{2} \cdot \bar{q} \rightarrow \hbar \bar{q}^{2} / 2$, and where the last line arises from averaging over the two equivalent expressions for $B_{-1}^{\mu}$.

We may similarly simplify the singular terms from the box and cross box. Indeed, using the identity (5.19) followed by the linear change of variable we have,

$$
\begin{aligned}
B_{-1}+C_{-1} & =-\frac{4 Q_{1}^{2} Q_{2}^{2}}{\hbar^{2+2 \epsilon}}\left(p_{1} \cdot p_{2}\right)^{2} \int \frac{\hat{d}^{D} \bar{\ell}}{\bar{\ell}^{2}(\bar{\ell}-\bar{q})^{2}} \frac{1}{\left(p_{1} \cdot \bar{\ell}+i \epsilon\right)} \hat{\delta}\left(p_{2} \cdot \bar{\ell}\right) \\
& =\frac{4 Q_{1}^{2} Q_{2}^{2}}{\hbar^{2+2 \epsilon}}\left(p_{1} \cdot p_{2}\right)^{2} \int \frac{\hat{d}^{D} \bar{\ell}^{\prime}}{\bar{\ell}^{\prime 2}\left(\bar{\ell}^{\prime}-\bar{q}\right)^{2}} \frac{1}{\left(p_{1} \cdot \bar{\ell}^{\prime}+\hbar \bar{q}^{2} / 2-i \epsilon\right)} \hat{\delta}\left(p_{2} \cdot \bar{\ell}^{\prime}-\hbar \bar{q}^{2} / 2\right) \\
& =\frac{2 i Q_{1}^{2} Q_{2}^{2}}{\hbar^{2}}\left(p_{1} \cdot p_{2}\right)^{2} \int \frac{\hat{d}^{4} \bar{\ell} \hat{\delta}\left(p_{1} \cdot \bar{\ell}\right) \hat{\delta}\left(p_{2} \cdot \bar{\ell}\right)}{\bar{\ell}^{2}(\bar{\ell}-\bar{q})^{2}}+\mathcal{O}(1 / \hbar)
\end{aligned}
$$

where we have averaged over equivalent forms, and then used eq. (5.19) a second time in obtaining the last line. At the very end, we took $D \rightarrow 4$. 
Combining eqs. (5.30) and (5.31), we find that the potentially singular contributions to the impulse kernel in the classical limit are,

$$
\begin{aligned}
& \left.\mathcal{I}_{\mathrm{cl}}^{\mu,(1)}\right|_{\text {singular }}=\hbar \bar{q}^{\mu}\left(B_{-1}+C_{-1}\right)+\hbar B_{-1}^{\mu} \\
& \quad=\frac{2 i Q_{1}^{2} Q_{2}^{2}}{\hbar}\left(p_{1} \cdot p_{2}\right)^{2} \bar{q}^{\mu}\left[\int \frac{\hat{d}^{4} \bar{\ell} \hat{\delta}\left(p_{1} \cdot \bar{\ell} \hat{\delta}\left(p_{2} \cdot \bar{\ell}\right)\right.}{\bar{\ell}^{2}(\bar{\ell}-\bar{q})^{2}}-\int \hat{d}^{4} \bar{\ell} \frac{\hat{\delta}\left(p_{1} \cdot \bar{\ell}\right) \hat{\delta}\left(p_{2} \cdot \bar{\ell}\right)}{\bar{\ell}^{2}(\bar{\ell}-\bar{q})^{2}}\right]+\mathcal{O}\left(\hbar^{0}\right) \\
& \quad=\mathcal{O}\left(\hbar^{0}\right) .
\end{aligned}
$$

The dangerous terms cancel, leaving only well-defined contributions.

It remains to extract the $\mathcal{O}(1 / \hbar)$ terms from the box, crossed box, and cut box contributions, and to combine them with the triangles (5.22), which are of this order. In addition to $B_{0}$ from eq. (5.24), $C_{0}$ from eq. (5.26), and $B_{0}^{\mu}$ from eq. (5.29), we must also include the $\mathcal{O}(1 / \hbar)$ terms left implicit in eqs. (5.30) and (5.31). In the former contributions, we can now set $p_{1,2} \cdot \bar{q}=0$, as the $\hbar$ terms in the on-shell delta functions would give rise to contributions of $\mathcal{O}\left(\hbar^{0}\right)$ to the impulse kernel, which in turn will give contributions of $\mathcal{O}(\hbar)$ to the impulse. In combining all these terms, we make use of summing over an expression and the expression after the linear change of variables; the identity (5.19); and the identity,

$$
\hat{\delta}^{\prime}(x)=\frac{i}{(x-i \epsilon)^{2}}-\frac{i}{(x+i \epsilon)^{2}} .
$$

We find that,

$$
\begin{aligned}
& \hbar \bar{q}^{\mu}\left(B_{0}+C_{0}\right)+\left.\left[\hbar \bar{q}^{\mu}\left(B_{-1}+C_{-1}\right)\right]\right|_{\mathcal{O}\left(\hbar^{0}\right)}= \\
& 2 Q_{1}^{2} Q_{2}^{2}\left(p_{1} \cdot p_{2}\right)^{2} \bar{q}^{\mu} \\
& \quad \times \int \frac{\hat{d}^{4} \bar{\ell}}{\bar{\ell}^{2}(\bar{\ell}-\bar{q})^{2}}\left(\hat{\delta}\left(p_{2} \cdot \bar{\ell}\right) \frac{\bar{\ell} \cdot(\bar{\ell}-\bar{q})}{\left(p_{1} \cdot \bar{\ell}+i \epsilon\right)^{2}}+\hat{\delta}\left(p_{1} \cdot \bar{\ell}\right) \frac{\bar{\ell} \cdot(\bar{\ell}-\bar{q})}{\left(p_{2} \cdot \bar{\ell}-i \epsilon\right)^{2}}\right)+Z^{\mu}, \\
& \hbar B_{0}^{\mu}+\left.\left[\hbar B_{-1}^{\mu}\right]\right|_{\mathcal{O}\left(\hbar^{0}\right)}= \\
& \quad-2 i Q_{1}^{2} Q_{2}^{2}\left(p_{1} \cdot p_{2}\right)^{2} \\
& \quad \times \int \frac{\hat{d}^{4} \bar{\ell}}{\bar{\ell}^{2}(\bar{\ell}-\bar{q})^{2}} \bar{\ell}^{\mu} \bar{\ell} \cdot(\bar{\ell}-\bar{q})\left(\hat{\delta}^{\prime}\left(p_{1} \cdot \bar{\ell}\right) \hat{\delta}\left(p_{2} \cdot \bar{\ell}\right)-\hat{\delta}^{\prime}\left(p_{2} \cdot \bar{\ell}\right) \hat{\delta}\left(p_{1} \cdot \bar{\ell}\right)\right)-Z^{\mu},
\end{aligned}
$$

where we have now taken $D \rightarrow 4$, and where the quantity $Z^{\mu}$ is,

$$
\begin{aligned}
Z^{\mu}= & i Q_{1}^{2} Q_{2}^{2}\left(p_{1} \cdot p_{2}\right)^{2} \bar{q}^{\mu} \\
& \times \int \frac{\hat{d}^{4} \bar{\ell}}{\bar{\ell}^{2}(\bar{\ell}-\bar{q})^{2}}\left(2 \bar{\ell} \cdot \bar{q}-\bar{\ell}^{2}\right)\left(\hat{\delta}^{\prime}\left(p_{1} \cdot \bar{\ell}\right) \hat{\delta}\left(p_{2} \cdot \bar{\ell}\right)-\hat{\delta}^{\prime}\left(p_{2} \cdot \bar{\ell}\right) \hat{\delta}\left(p_{1} \cdot \bar{\ell}\right)\right) .
\end{aligned}
$$

Finally, we integrate over the external wavefunctions. The possible singularity in $\hbar$ has canceled, so as discussed in section 4.1, we perform the integrals by replacing the momenta $p_{i}$ with their classical values $m_{i} u_{i}$, so that the box-derived contribution is,

$$
\begin{aligned}
\mathcal{I}_{2}^{\mu} \equiv & 2 Q_{1}^{2} Q_{2}^{2} \gamma^{2} \bar{q}^{\mu} \\
& \times \int \frac{\hat{d}^{4} \bar{\ell}}{\bar{\ell}^{2}(\bar{\ell}-\bar{q})^{2}}\left(m_{2} \hat{\delta}\left(u_{2} \cdot \bar{\ell}\right) \frac{\bar{\ell} \cdot(\bar{\ell}-\bar{q})}{\left(u_{1} \cdot \bar{\ell}+i \epsilon\right)^{2}}+m_{1} \hat{\delta}\left(u_{1} \cdot \bar{\ell}\right) \frac{\bar{\ell} \cdot(\bar{\ell}-\bar{q})}{\left(u_{2} \cdot \bar{\ell}-i \epsilon\right)^{2}}\right),
\end{aligned}
$$


while that from the cut box is,

$$
\mathcal{I}_{3}^{\mu} \equiv-2 i Q_{1}^{2} Q_{2}^{2} \gamma^{2} \int \frac{\hat{d}^{4} \bar{\ell}}{\bar{\ell}^{2}(\bar{\ell}-\bar{q})^{2}} \bar{\ell}^{\mu} \bar{\ell} \cdot(\bar{\ell}-\bar{q})\left(m_{2} \hat{\delta}^{\prime}\left(u_{1} \cdot \bar{\ell}\right) \hat{\delta}\left(u_{2} \cdot \bar{\ell}\right)-m_{1} \hat{\delta}^{\prime}\left(u_{2} \cdot \bar{\ell}\right) \hat{\delta}\left(u_{1} \cdot \bar{\ell}\right)\right)
$$

In both contributions, we have dropped the $Z^{\mu}$ term which cancels between the two. The full impulse kernel is given by the sum $\mathcal{I}_{1}^{\mu}+\mathcal{I}_{2}^{\mu}+\mathcal{I}_{3}^{\mu}$, and the impulse by,

$$
\begin{aligned}
\Delta p_{1}^{\mu,(1)}= & \frac{i e^{4}}{4} \hbar \int \hat{d}^{4} \bar{q} \hat{\delta}\left(\bar{q} \cdot u_{1}\right) \hat{\delta}\left(\bar{q} \cdot u_{2}\right) e^{-i \bar{q} \cdot b} \mathcal{I}_{\mathrm{cl}}^{\mu,(1)} \\
= & \frac{i e^{4}}{4} \hbar \int \hat{d}^{4} \bar{q} \hat{\delta}\left(\bar{q} \cdot u_{1}\right) \hat{\delta}\left(\bar{q} \cdot u_{2}\right) e^{-i \bar{q} \cdot b}\left(\mathcal{I}_{1}^{\mu}+\mathcal{I}_{2}^{\mu}+\mathcal{I}_{3}^{\mu}\right) \\
= & \frac{i Q_{1}^{2} Q_{2}^{2} e^{4}}{2} \int \frac{\hat{d}^{4} \bar{\ell}}{\overline{\ell^{2}}(\bar{\ell}-\bar{q})^{2}} \hat{d}^{4} \bar{q} \hat{\delta}\left(\bar{q} \cdot u_{1}\right) \hat{\delta}\left(\bar{q} \cdot u_{2}\right) e^{-i \bar{q} \cdot b} \\
& \times\left[\bar{q}^{\mu}\left(\frac{\hat{\delta}\left(u_{1} \cdot \bar{\ell}\right)}{m_{2}}+\frac{\hat{\delta}\left(u_{2} \cdot \bar{\ell}\right)}{m_{1}}\right)\right. \\
& +\gamma^{2} \bar{q}^{\mu}\left(\frac{\hat{\delta}\left(u_{2} \cdot \bar{\ell}\right)}{m_{1}} \frac{\bar{\ell} \cdot(\bar{\ell}-\bar{q})}{\left(u_{1} \cdot \bar{\ell}+i \epsilon\right)^{2}}+\frac{\hat{\delta}\left(u_{1} \cdot \bar{\ell}\right)}{m_{2}} \frac{\bar{\ell} \cdot(\bar{\ell}-\bar{q})}{\left(u_{2} \cdot \bar{\ell}-i \epsilon\right)^{2}}\right) \\
& \left.-i \gamma^{2} \bar{\ell}^{\mu} \bar{\ell} \cdot(\bar{\ell}-\bar{q})\left(\frac{\hat{\delta}^{\prime}\left(u_{1} \cdot \bar{\ell}\right) \hat{\delta}\left(u_{2} \cdot \bar{\ell}\right)}{m_{1}}-\frac{\hat{\delta}^{\prime}\left(u_{2} \cdot \bar{\ell}\right) \hat{\delta}\left(u_{1} \cdot \bar{\ell}\right)}{m_{2}}\right)\right] .
\end{aligned}
$$

\subsubsection{On-shell cross check}

As we have seen, careful inclusion of boxes, crossed boxes as well as cut boxes are necessary to determine the impulse in the classical regime. This may seem to be at odds with other work on the classical limit of amplitudes, which often emphasises the particular importance of triangle diagrams to the classical potential at next to leading order. However, in the context of the potential, the partial cancellation between boxes and crossed boxes is well-understood [79], and it is because of this fact that triangle diagrams are particularly important. The residual phase is known to exponentiate so that it does not effect classical physics. Meanwhile, the relevance of the subtraction of iterated (cut) diagrams has been discussed in $[69,81,136]$.

Nevertheless in the case of the impulse it may seem that the various boxes play a more significant role, as they certainly contribute to the classical result for the impulse. In fact, it is easy to see that these terms must be included to recover a physically sensible result. The key observation is that the final momentum, $r_{1}^{\mu}$, of the outgoing particle after a classical scattering process must be on shell, $r_{1}^{2}=m_{1}^{2}$.

We may express the final momentum in terms of the initial momentum and the impulse, so that

$$
r_{1}^{\mu}=p_{1}^{\mu}+\Delta p_{1}^{\mu}
$$

The on-shell condition is then

$$
\left(\Delta p_{1}\right)^{2}+2 p_{1} \cdot \Delta p_{1}=0
$$


At order $e^{2}$, this requirement is satisfied trivially. At this order $\left(\Delta p_{1}\right)^{2}$ is negligible, while

$$
p_{1} \cdot \Delta p_{1}=i m_{1} e^{2} Q_{1} Q_{2} \int \hat{d}^{4} \bar{q} \hat{\delta}\left(\bar{q} \cdot u_{1}\right) \hat{\delta}\left(\bar{q} \cdot u_{2}\right) e^{-i \bar{q} \cdot b} \bar{q} \cdot u_{1} \frac{u_{1} \cdot u_{2}}{\bar{q}^{2}}=0
$$

using our result for the $\mathrm{LO}$ impulse in eq. (5.4).

The situation is less trivial at order $e^{4}$, as neither $p_{1} \cdot \Delta p_{1}$ nor $\left(\Delta p_{1}\right)^{2}$ vanish. In fact, at this order we may use eq. (5.4) once again to find that

$$
\begin{aligned}
\left(\Delta p_{1}\right)^{2}= & -e^{4} Q_{1}^{2} Q_{2}^{2}\left(u_{1} \cdot u_{2}\right)^{2} \\
& \times \int \hat{d}^{4} \bar{q} \hat{d}^{4} \bar{q}^{\prime} \hat{\delta}\left(\bar{q} \cdot u_{1}\right) \hat{\delta}\left(\bar{q} \cdot u_{2}\right) \hat{\delta}\left(\bar{q}^{\prime} \cdot u_{1}\right) \hat{\delta}\left(\bar{q}^{\prime} \cdot u_{2}\right) e^{-i\left(\bar{q}+\bar{q}^{\prime}\right) \cdot b} \frac{\bar{q} \cdot \bar{q}^{\prime}}{\bar{q}^{2} \bar{q}^{\prime 2}} .
\end{aligned}
$$

Meanwhile, to evaluate $p_{1} \cdot \Delta p_{1}$ we must turn to our NLO result for the impulse, eq. (5.38). Thanks to the delta functions present in the impulse, we find a simple expression:

$$
\begin{aligned}
2 p_{1} \cdot \Delta p_{1}= & e^{4} Q_{1}^{2} Q_{2}^{2}\left(u_{1} \cdot u_{2}\right)^{2} \\
& \times \int \hat{d}^{4} \bar{q} \hat{\delta}\left(\bar{q} \cdot u_{1}\right) \hat{\delta}\left(\bar{q} \cdot u_{2}\right) e^{-i \bar{q} \cdot b} \int \hat{d}^{4} \bar{\ell} \bar{\ell} \cdot u_{1} \hat{\delta}^{\prime}\left(\bar{\ell} \cdot u_{1}\right) \hat{\delta}\left(\bar{\ell} \cdot u_{2}\right) \frac{\bar{\ell} \cdot(\bar{\ell}-\bar{q})}{\bar{\ell}^{2}(\bar{\ell}-\bar{q})^{2}} .
\end{aligned}
$$

To simplify this expression, it may be helpful to imagine working in the restframe of the timelike vector $u_{1}$. Then, the $\bar{\ell}$ integral involves the distribution $\bar{\ell}_{0} \hat{\delta}^{\prime}\left(\bar{\ell}_{0}\right)$, while $\bar{q}_{0}=0$. Thus the $\bar{\ell}_{0}$ integral has the form

$$
\int \hat{d} \bar{\ell}_{0} \bar{\ell}_{0} \hat{\delta}^{\prime}\left(\bar{\ell}_{0}\right) f\left(\bar{\ell}_{0}^{2}\right)=-\int \hat{d} \bar{\ell}_{0} \hat{\delta}\left(\bar{\ell}_{0}\right) f\left(\bar{\ell}_{0}^{2}\right) .
$$

Using this observation, we may simplify equation (5.43) to find

$$
\begin{aligned}
2 p_{1} \cdot \Delta p_{1}= & -e^{4} Q_{1}^{2} Q_{2}^{2}\left(u_{1} \cdot u_{2}\right)^{2} \\
& \times \int \hat{d}^{4} \bar{q} \hat{d}^{4} \bar{\ell} \hat{\delta}\left(\bar{q} \cdot u_{1}\right) \hat{\delta}\left(\bar{q} \cdot u_{2}\right) \hat{\delta}\left(\bar{\ell} \cdot u_{1}\right) \hat{\delta}\left(\bar{\ell} \cdot u_{2}\right) e^{-i \bar{q} \cdot b} \overline{\bar{\ell} \cdot(\bar{\ell}-\bar{q})} \\
= & e^{4} Q_{1}^{2} Q_{2}^{2}\left(u_{1} \cdot u_{2}\right)^{2} \\
& \times \int \hat{d}^{4} \bar{\ell} \hat{d}^{4} \bar{q}^{\prime} \hat{\delta}\left(\bar{\ell} \cdot u_{1}\right) \hat{\delta}\left(\bar{\ell} \cdot u_{2}\right) \hat{\delta}\left(\bar{q}^{\prime} \cdot u_{1}\right) \hat{\delta}\left(\bar{q}^{\prime} \cdot u_{2}\right) e^{-i\left(\bar{\ell}+\bar{q}^{\prime}\right) \cdot b} \frac{\bar{\ell} \cdot \bar{q}^{\prime}}{\bar{\ell}^{2} \bar{q}^{\prime 2}},
\end{aligned}
$$

where in the last line we set $\bar{q}^{\prime}=\bar{q}-\bar{\ell}$. This expression is equal but opposite to eq. (5.42), and so the final momentum is on shell as it must be.

It is worth remarking that the part of the NLO impulse that is relevant in this cancellation arises solely from the cut boxes. One can therefore view this phenomenon as an analogue of the removal of iterations of the tree in the potential.

\subsection{Radiation}

The LO and NLO impulse are conservative in the sense that momentum is simply exchanged from particle 1 to particle 2 at these orders; it is only at NNLO that momentum radiated away back-reacts on the impulse. We will study this back-reaction in the next section, but first we turn to a direct computation of the radiated momentum. 


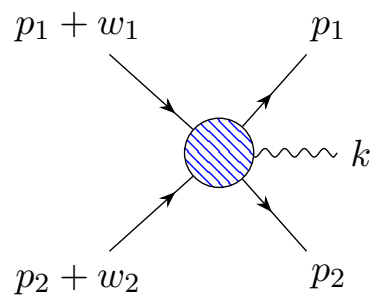

Figure 1. The amplitude $\mathcal{A}^{(0)}\left(p_{1}+w_{1}, p_{2}+w_{2} \rightarrow p_{1}, p_{2}, k\right)$ appearing in the radiation kernel at leading order.

The relevance of a classical limit of a scattering amplitude to what we are calling the radiation kernel was previously discussed by one of the authors and his collaborators [137]. The main advantage of the present discussion of radiation is that we have constructed a first-principles definition of the radiated momentum eq. (4.41) in terms of an on-shell scattering amplitude, eq. (4.40). Our goal in this section is to compute the LO radiation kernel explicitly in electrodynamics. Later, in section 6.2 .2 , we will see that the radiation kernel has the classical interpretation of a current.

The amplitude appearing in the radiation kernel is a five-point, tree amplitude (figure 1) which is easily computed. We find that the radiation kernel is,

$$
\begin{aligned}
\mathcal{R}^{(0)}(\bar{k})= & 4 \int \hat{d}^{4} \bar{w}_{1} \hat{d}^{4} \bar{w}_{2} \hat{\delta}\left(2 p_{1} \cdot \bar{w}_{1}\right) \hat{\delta}\left(2 p_{2} \cdot \bar{w}_{2}\right) \hat{\delta}^{(4)}\left(\bar{k}-\bar{w}_{1}-\bar{w}_{2}\right) e^{i \bar{w}_{1} \cdot b} \\
& \times\left\{\frac { Q _ { 1 } ^ { 2 } Q _ { 2 } } { \overline { w } _ { 2 } ^ { 2 } } \left[-p_{2} \cdot \varepsilon+\frac{\left(p_{1} \cdot p_{2}\right)\left(\bar{w}_{2} \cdot \varepsilon\right)}{p_{1} \cdot \bar{k}}+\frac{\left(p_{2} \cdot \bar{k}\right)\left(p_{1} \cdot \varepsilon\right)}{p_{1} \cdot \bar{k}}\right.\right. \\
& \left.\left.-\frac{\left(\bar{k} \cdot \bar{w}_{2}\right)\left(p_{1} \cdot p_{2}\right)\left(p_{1} \cdot \varepsilon\right)}{\left(p_{1} \cdot \bar{k}\right)^{2}}\right]+(1 \leftrightarrow 2)\right\},
\end{aligned}
$$

where $\varepsilon$ is the polarization vector for the emitted photon. As the quantities $p_{i} \cdot \bar{k}$ do not vanish on the support of the integrals in the radiation kernel, we have ignored the $i \epsilon$ factors in the massive propagators.

This lowest order radiation kernel is of $\mathcal{O}\left(\hbar^{0}\right)$, so we may now perform the integrals over the particle wavefunctions, which effectively replaces the momenta $p_{i}$ in the radiation kernel by their classical values $m_{i} u_{i}$.

$$
\begin{aligned}
\mathcal{R}^{(0)}(\bar{k}) \rightarrow & \frac{1}{m_{1}} \int \hat{d}^{4} \bar{w}_{1} \hat{d}^{4} \bar{w}_{2} \hat{\delta}\left(u_{1} \cdot \bar{w}_{1}\right) \hat{\delta}\left(u_{2} \cdot \bar{w}_{2}\right) \hat{\delta}^{(4)}\left(\bar{k}-\bar{w}_{1}-\bar{w}_{2}\right) e^{i \bar{w}_{1} \cdot b} \\
& \times\left\{\frac { Q _ { 1 } ^ { 2 } Q _ { 2 } } { \overline { w } _ { 2 } ^ { 2 } } \left[-u_{2} \cdot \varepsilon+\frac{\left(u_{1} \cdot u_{2}\right)\left(\bar{w}_{2} \cdot \varepsilon\right)}{u_{1} \cdot \bar{k}}+\frac{\left(u_{2} \cdot \bar{k}\right)\left(u_{1} \cdot \varepsilon\right)}{u_{1} \cdot \bar{k}}\right.\right. \\
& \left.\left.-\frac{\left(\bar{k} \cdot \bar{w}_{2}\right)\left(u_{1} \cdot u_{2}\right)\left(u_{1} \cdot \varepsilon\right)}{\left(u_{1} \cdot \bar{k}\right)^{2}}\right]+(1 \leftrightarrow 2)\right\}
\end{aligned}
$$

We will see this expression once again in section 6.2.2, appearing as a classical current. 


\subsection{Momentum conservation and radiation reaction}

We have already seen that conservation of momentum holds exactly (in section 3.5) and in our classical expressions (in section 4.5). Let us now make sure that there is no subtlety in these discussions by explicit calculation.

To do so, we calculate the part of the NNLO impulse $I_{(2), \text { cl }}^{\mu,(\mathrm{rad})}$ which encodes radiation reaction, defined in eq. (4.46). The two amplitudes appearing in equation (4.46) are in common with the amplitudes relevant for the radiated momentum, equation (4.42), though they are evaluated at slightly different kinematics. It will be convenient to change the sign of $\bar{w}_{i}$ here; with that change, the amplitudes are:

$$
\begin{aligned}
& \overline{\mathcal{A}}^{(0)}\left(p_{1} p_{2} \rightarrow p_{1}-\hbar \bar{w}_{1}, p_{2}-\hbar \bar{w}_{2}, \bar{k}\right)= \\
& \quad \frac{4 Q_{1}^{2} Q_{2}}{\hbar^{2} \bar{w}_{2}^{2}}\left[-p_{2} \cdot \varepsilon+\frac{\left(p_{1} \cdot p_{2}\right)\left(\bar{w}_{2} \cdot \varepsilon\right)}{p_{1} \cdot \bar{k}}+\frac{\left(p_{2} \cdot \bar{k}\right)\left(p_{1} \cdot \varepsilon\right)}{p_{1} \cdot \bar{k}}-\frac{\left(\bar{k} \cdot \bar{w}_{2}\right)\left(p_{1} \cdot p_{2}\right)\left(p_{1} \cdot \varepsilon\right)}{\left(p_{1} \cdot \bar{k}\right)^{2}}\right] \\
& \quad+(1 \leftrightarrow 2),
\end{aligned}
$$

and

$$
\begin{aligned}
& \overline{\mathcal{A}}^{(0) *}\left(p_{1}+\hbar \bar{q}_{1}, p_{2}+\hbar \bar{q}_{2} \rightarrow p_{1}-\hbar \bar{w}_{1}, p_{2}-\hbar \bar{w}_{2}, \bar{k}\right)= \\
& \quad \frac{4 Q_{1}^{2} Q_{2}}{\hbar^{2} \bar{w}_{2}^{\prime 2}}\left[-p_{2} \cdot \varepsilon^{*}+\frac{\left(p_{1} \cdot p_{2}\right)\left(\bar{w}_{2}^{\prime} \cdot \varepsilon^{*}\right)}{p_{1} \cdot \bar{k}}+\frac{\left(p_{2} \cdot \bar{k}\right)\left(p_{1} \cdot \varepsilon^{*}\right)}{p_{1} \cdot \bar{k}}-\frac{\left(\bar{k} \cdot \bar{w}_{2}^{\prime}\right)\left(p_{1} \cdot p_{2}\right)\left(p_{1} \cdot \varepsilon^{*}\right)}{\left(p_{1} \cdot \bar{k}\right)^{2}}\right] \\
& \quad+(1 \leftrightarrow 2),
\end{aligned}
$$

where we find it convenient to define $\bar{w}_{i}^{\prime}=\bar{q}_{i}+\bar{w}_{i}$ (after the change of sign).

We can now write the impulse contribution as,

$$
I_{(2), \mathrm{cl}}^{\mu,(\mathrm{rad})}=-e^{6}\left\langle\left\langle\int d \Phi(\bar{k}) \prod_{i=1,2} \int \hat{d}^{4} \bar{w}_{i} \hat{d}^{4} \bar{w}_{i}^{\prime} \bar{w}_{1}^{\mu} \mathcal{X}\left(\bar{w}_{1}, \bar{w}_{2}, \bar{k}\right) \mathcal{X}^{*}\left(\bar{w}_{1}^{\prime}, \bar{w}_{2}^{\prime}, \bar{k}\right)\right\rangle\right\rangle,
$$

where

$$
\begin{aligned}
\mathcal{X}\left(\bar{w}_{1}, \bar{w}_{2}, \bar{k}\right)= & 4 \hat{\delta}\left(2 \bar{w}_{1} \cdot p_{1}\right) \hat{\delta}\left(2 \bar{w}_{2} \cdot p_{2}\right) \hat{\delta}^{(4)}\left(\bar{k}-\bar{w}_{1}-\bar{w}_{2}\right) e^{i b \cdot \bar{w}_{1}} \\
& \times\left\{Q _ { 1 } ^ { 2 } Q _ { 2 } \frac { \varepsilon _ { \mu } } { \overline { w } _ { 2 } ^ { 2 } } \left[-p_{2}^{\mu}+\frac{p_{1} \cdot p_{2} \bar{w}_{2}^{\mu}}{p_{1} \cdot \bar{k}}+\frac{p_{2} \cdot \bar{k} p_{1}^{\mu}}{p_{1} \cdot \bar{k}}\right.\right. \\
& \left.\left.-\frac{\left(\bar{k} \cdot \bar{w}_{2}\right)\left(p_{1} \cdot p_{2}\right) p_{1}^{\mu}}{\left(p_{1} \cdot \bar{k}\right)^{2}}\right]+(1 \leftrightarrow 2)\right\} .
\end{aligned}
$$

This expression is directly comparable to those for radiated momentum: eq. (5.50), and the equivalent impulse contribution to particle 2, balance the radiated momentum eq. (4.43) using $\bar{w}_{1}^{\mu}+\bar{w}_{2}^{\mu}=\bar{k}^{\mu}$, provided that the radiation kernel, eq. (5.46), is related to integrals over $\mathcal{X}$. Indeed this relationship holds: the integrations present in the radiation kernel are supplied by the $\bar{w}_{i}$ and $\bar{w}_{i}^{\prime}$ integrals in eq. (5.50); these integrations disentangle in the sum of impulses on particles 1 and 2 when we impose $\bar{w}_{1}^{\mu}+\bar{w}_{2}^{\mu}=\bar{k}^{\mu}$, and then form the square of the radiation kernel.

It is interesting to compare this radiated momentum with the situation in traditional formulations of classical physics, where one must include the ALD radiation reaction force by hand in order to enforce momentum conservation. Because the situation is simplest 
when only one particle is dynamical, let us take the mass $m_{2}$ to be very large compared to $m_{1}$ in the remainder of this section, and work in particle 2's rest frame. In this frame, it does not radiate, and the only radiation reaction is on particle 1 - the radiated momentum is precisely balanced by the impulse on particle 1 due to the ALD force. We can therefore continue our discussion with reference to our expression for radiated momentum, eq. (4.43) and the radiation kernel, eq. (5.46). In this situation we may also simplify the kernels by dropping the $(1 \leftrightarrow 2)$ instruction: notice that the explicit terms in the kernel of equation (5.46) are linear in $m_{2}$. Terms obtained by symmetrising in particle labels are therefore linear in $m_{1}$, and so are subdominant when $m_{2} \gg m_{1}$.

We will compute the impulse due to the ALD force directly from its classical expression in section 6.3. But in preparation for that comparison there is one step which we must take. Classical expressions for the force - which involve only the particle's momentum and its derivatives - do not involve any photon phase space. So we must perform the integration over $d \Phi(\bar{k})$ which is present in eq. (4.43).

To organise the calculation, we integrate over the $\bar{q}_{1}$ variables in the radiation kernel, eq. (5.46) using the four-fold delta function, so that we may write the radiated momentum as,

$$
R_{\mathrm{cl}}^{\mu,(0)}=-\frac{e^{6} Q_{1}^{4} Q_{2}^{2}}{m_{1}^{2}} \int \hat{d}^{4} \bar{q} \hat{d}^{4} \bar{q}^{\prime} e^{-i b \cdot\left(\bar{q}-\bar{q}^{\prime}\right)} \hat{\delta}\left(u_{1} \cdot\left(\bar{q}-\bar{q}^{\prime}\right)\right) \frac{\hat{\delta}\left(u_{2} \cdot \bar{q}\right)}{\bar{q}^{2}} \frac{\hat{\delta}\left(u_{2} \cdot \bar{q}^{\prime}\right)}{\bar{q}^{\prime 2}} Y_{\mathrm{r}}^{\mu},
$$

where we renamed the remaining variables, $\bar{w}_{2} \rightarrow \bar{q}$ and $\bar{w}_{2}^{\prime} \rightarrow \bar{q}^{\prime}$, in order to match the notation used later in section 6 . After some algebra we find,

$$
\begin{aligned}
Y_{\mathrm{r}}^{\mu}= & \int d \Phi(\bar{k}) \hat{\delta}\left(u_{1} \cdot \bar{k}-\bar{E}\right) \bar{k}^{\mu}\left[1+\frac{\left(u_{1} \cdot u_{2}\right)^{2}\left(\bar{q} \cdot \bar{q}^{\prime}\right)}{\bar{E}^{2}}+\frac{\left(u_{2} \cdot \bar{k}\right)^{2}}{\bar{E}^{2}}\right. \\
& \left.-\frac{\left(u_{1} \cdot u_{2}\right)\left(u_{2} \cdot \bar{k}\right) \bar{k} \cdot\left(\bar{q}+\bar{q}^{\prime}\right)}{\bar{E}^{3}}+\frac{\left(u_{1} \cdot u_{2}\right)^{2}(\bar{k} \cdot \bar{q})\left(\bar{k} \cdot \bar{q}^{\prime}\right)}{\bar{E}^{4}}\right] .
\end{aligned}
$$

The quantity $\bar{E}$ is defined to be $\bar{E}=u_{1} \cdot \bar{k}$; in view of the delta function, the integral is constrained so that $\bar{E}=u_{1} \cdot \bar{q}$. This quantity is the wavenumber of the photon in the rest frame of particle 1 , and is fixed from the point of view of the phase space integration. As a result, the integrals are simple: there are two delta functions (one explicit, one in the phase space measure) which can be used to perform the $\bar{k}^{0}$ integration and to fix the magnitude of the spatial wavevector. The remaining integrals are over angles and are performed in appendix $\mathrm{C}$. The radiated momentum takes a remarkably simple form after the phase space integration:

$$
\begin{aligned}
R_{\mathrm{cl}}^{\mu,(0)}= & -\frac{e^{6} Q_{1}^{4} Q_{2}^{2}}{3 \pi m_{1}^{2}} \int \hat{d}^{4} \bar{q} \hat{d}^{4} \bar{q}^{\prime} e^{-i b \cdot\left(\bar{q}-\bar{q}^{\prime}\right)} \hat{\delta}\left(u_{1} \cdot\left(\bar{q}-\bar{q}^{\prime}\right)\right) \frac{\hat{\delta}\left(u_{2} \cdot \bar{q}\right)}{\bar{q}^{2}} \frac{\hat{\delta}\left(u_{2} \cdot \bar{q}^{\prime}\right)}{\bar{q}^{\prime 2}} \Theta\left(u_{1} \cdot \bar{q}\right) \\
& \times\left[\left(u_{1} \cdot \bar{q}\right)^{2}+\bar{q} \cdot \bar{q}^{\prime}\left(u_{1} \cdot u_{2}\right)^{2}\right] u_{1}^{\mu} .
\end{aligned}
$$

The $\Theta$ function is a remnant of the photon phase space volume, so it will be convenient to remove it. The delta functions in the integrand in eq. (5.54) constrain the components of the vectors $\bar{q}$ and $\bar{q}^{\prime}$ which lie in the two dimensional space spanned by $u_{1}$ and $u_{2}$. Let 
us call the components of $q$ and $q^{\prime}$ in this plane to be $q_{\|}$and $q_{\|}^{\prime}$. Then the delta functions set $q_{\|}=q_{\|}^{\prime}$. As a result, the integrand (ignoring the $\Theta$ function) is symmetric in $q_{\|} \rightarrow-q_{\|}$. Consequently we may symmetrise to find

$$
\begin{aligned}
R_{\mathrm{cl}}^{\mu,(0)}= & -\frac{e^{6} Q_{1}^{4} Q_{2}^{2}}{6 \pi m_{1}^{2}} \int \hat{d}^{4} \bar{q} \hat{d}^{4} \bar{q}^{\prime} e^{-i b \cdot\left(\bar{q}-\bar{q}^{\prime}\right)} \hat{\delta}\left(u_{1} \cdot\left(\bar{q}-\bar{q}^{\prime}\right)\right) \frac{\hat{\delta}\left(u_{2} \cdot \bar{q}\right)}{\bar{q}^{2}} \frac{\hat{\delta}\left(u_{2} \cdot \bar{q}^{\prime}\right)}{\bar{q}^{\prime 2}} \\
& \times\left[\left(u_{1} \cdot \bar{q}\right)^{2}+\bar{q} \cdot \bar{q}^{\prime}\left(u_{1} \cdot u_{2}\right)^{2}\right] u_{1}^{\mu} .
\end{aligned}
$$

We will see in section 6.3 that this expression is equal but opposite to the impulse obtained from the classical ALD force.

\section{Classical calculations}

In previous sections, we have shown how to understand the electromagnetic scattering of point-like particles $Q_{i}$ using the methods of quantum field theory. We now turn to a purely classical approach to the same observables, restricting (once again) to electromagnetic scattering for simplicity. Our goal in this section is to reassure any skeptical reader that the expressions which we have claimed to be classical, are indeed classical. This is straightforward in electrodynamics; it would be harder in gravity. We expect, however, that an application of our methods in a gravitational context will be advantageous.

As usual we have in mind a two-body collision, and we discuss, firstly, the impulse on a particle, secondly the momentum radiated away, and lastly the topic of conservation of momentum and the ALD radiation reaction force. Our classical approach will be to solve the coupled equations of motion perturbatively. As we will see, these calculations have an iterative structure. This iteration is straightforward in principle, though it quickly becomes tedious in practice. For this reason we will frequently restrict to the case where the mass $m_{2}$ of particle 2 is much larger than the mass $m_{1}$ of particle 1 , so that particle 2 can be treated as being static. This assumption was unnecessary using quantum methods, ${ }^{4}$ where the symmetry of Feynman diagrams simplifies matters.

\subsection{The classical electromagnetic impulse}

Classically, we may take our particles to move along world-lines $x_{i}\left(\tau_{i}\right)$ with proper velocities $v_{i}\left(\tau_{i}\right)=d x_{i} / d \tau_{i}$ and momenta $p_{i}\left(\tau_{i}\right)=m_{i} v_{i}\left(\tau_{i}\right)$. We must solve the Maxwell equation,

$$
\partial_{\mu} F^{\mu \nu}(x)=J^{\nu}(x)=e \sum_{i} Q_{i} \int d \tau_{i} \delta^{4}\left(x-x_{i}\left(\tau_{i}\right)\right) v_{i}^{\nu}\left(\tau_{i}\right),
$$

and the Lorentz force laws, ${ }^{5}$

$$
\frac{d p_{i}^{\mu}}{d \tau_{i}}=e Q_{i} F^{\mu \nu}\left(x_{i}\left(\tau_{i}\right)\right) v_{i \nu}\left(\tau_{i}\right)
$$

\footnotetext{
${ }^{4}$ With the exception of radiation reaction - in that case, we took $m_{2}$ large to prepare for a comparison with classical results.

${ }^{5}$ We will need to include the ALD force law later to account for radiation reaction.
} 
To set up the perturbative method, we assume that the trajectories can be expanded as a power series in the coupling. At zeroth order in the expansion, the trajectories are simply straight lines:

$$
x_{1}\left(\tau_{1}\right)=b+u_{1} \tau_{1}, \quad x_{2}\left(\tau_{2}\right)=u_{2} \tau_{2},
$$

where $u_{1}$ and $u_{2}$ are constant vectors. Note that, as with our quantum mechanical setup in eq. (3.1), we have aligned the initial trajectory of particle 2 with the spatial origin, and have translated particle 1 through an impact parameter $b$ relative to particle 2 . The vectors $u_{i}$ are the zeroth order terms in the proper velocities: $v_{i}\left(\tau_{i}\right)=u_{i}+\mathcal{O}\left(e^{2}\right)$; they will play a prominent role below.

The perturbative expansion proceeds by determining the first order electromagnetic field sourced by the two particles, taken to be moving on straight-line trajectories. Knowledge of the field allows us to compute the first order forces on the particles, and hence the associated (small) first order deviations of the trajectories from straight line motion. Armed with this knowledge of the first order trajectory, we may compute the second order fields, forces and deviations. Iterating this procedure allows us to compute to any desired perturbative order. More formally, we expand the trajectories as,

$$
\begin{aligned}
& x_{1}(\tau)=b+u_{1} \tau+\Delta^{(1)} x_{1}(\tau)+\Delta^{(2)} x_{1}(\tau)+\cdots, \\
& x_{2}(\tau)=u_{2} \tau+\Delta^{(1)} x_{2}(\tau)+\Delta^{(2)} x_{2}(\tau)+\cdots,
\end{aligned}
$$

where $\Delta^{(n)} x(\tau)$ is of order $e^{2 n}$. We will similarly expand the velocities and forces perturbatively:

$$
\begin{aligned}
& v_{i}\left(\tau_{i}\right)=u_{i}+\Delta^{(1)} v_{i}\left(\tau_{i}\right)+\Delta^{(2)} v_{i}\left(\tau_{i}\right), \\
& f_{i}\left(\tau_{i}\right)=\Delta^{(1)} f_{i}\left(\tau_{i}\right)+\Delta^{(2)} f_{i}\left(\tau_{i}\right),
\end{aligned}
$$

where again $\Delta^{(n)} v_{i}\left(\tau_{i}\right)$ and $\Delta^{(n)} f_{i}\left(\tau_{i}\right)$ are of order $e^{2 n}$.

\subsubsection{Leading order}

Our first order of business is to determine the leading order electromagnetic fields. Classically, the force on particle 1 is due to the field of particle 2 ; as the result is symmetric under interchange of the two particles, we will only compute the field due to particle 2 . Working in Lorenz gauge, the relevant gauge field is,

$$
\partial^{2} A_{2}^{\mu}(x)=Q_{2} e \int d \tau \delta^{4}\left(x-u_{2} \tau\right) u_{2}^{\mu} .
$$

We find it convenient to work in Fourier space, choosing the conventions

$$
\begin{aligned}
& f(x)=\int \hat{d}^{4} \bar{q} \tilde{f}(\bar{q}) e^{-i \bar{q} \cdot x}, \\
& \tilde{f}(\bar{q})=\int d^{4} x f(x) e^{i \bar{q} \cdot x} .
\end{aligned}
$$


The momentum-space gauge field is easily found to be

$$
\widetilde{A}_{2}^{\mu}(\bar{q})=-\frac{e Q_{2}}{\bar{q}^{2}} u_{2}^{\mu} \hat{\delta}\left(\bar{q} \cdot u_{2}\right)
$$

with field strength (in position space)

$$
F_{2}^{\mu \nu}(x)=i e Q_{2} \int \hat{d}^{4} \bar{q} \hat{\delta}\left(\bar{q} \cdot u_{2}\right) e^{-i \bar{q} \cdot x} \frac{\bar{q}^{\mu} u_{2}^{\nu}-u_{2}^{\mu} \bar{q}^{\nu}}{\bar{q}^{2}} .
$$

The Lorentz force, equation (6.2), on particle 1 requires this field strength evaluated at the position of particle 1 . At leading order, we may use the straight line approximation to the trajectory of particle 1 , so that the leading order force is

$$
\frac{d p_{1}^{\mu}}{d \tau_{1}}=i e^{2} Q_{1} Q_{2} \int \hat{d}^{4} \bar{q} \hat{\delta}\left(\bar{q} \cdot u_{2}\right) e^{-i \bar{q} \cdot\left(b+u_{1} \tau_{1}\right)} \frac{\bar{q}^{\mu} u_{1} \cdot u_{2}-u_{2}^{\mu} \bar{q} \cdot u_{1}}{\bar{q}^{2}} .
$$

The leading order impulse is the total time integral of the force,

$$
\begin{aligned}
\Delta p_{1}^{\mu,(0)} & \equiv \int_{-\infty}^{\infty} d \tau_{1} \frac{d p_{1}^{\mu}}{d \tau_{1}} \\
& =i e^{2} Q_{1} Q_{2} \int \hat{d}^{4} \bar{q} \hat{\delta}\left(\bar{q} \cdot u_{1}\right) \hat{\delta}\left(\bar{q} \cdot u_{2}\right) e^{-i \bar{q} \cdot b} \bar{q}^{\mu} u_{1} \cdot u_{2} \frac{1}{\bar{q}^{2}}
\end{aligned}
$$

in complete agreement with eq. (5.4), which was obtained from a tree-level scattering amplitude.

\subsubsection{Next-to-leading order}

The LO calculation is very simple, and so it is more interesting to look at the next order of perturbation theory. In our quantum-mechanical treatment, this following order was computed in section 5.2 using loop diagrams. In this section, we derive the same expression by iterating the perturbative solution of the classical equations to the next order. The fact that both methods yield the same result is a vivid demonstration that loops involving massive particles are not simply quantum corrections [83].

In this section we take particle 2 to be static, leaving the more general case as an exercise for the reader. When particle 2 is static, its field strength is given by equation (6.11) to all orders.

The NLO impulse is the time integral of the NLO Lorentz force. With our assumption of a static particle 2, we know the gauge field acting on particle 1 exactly, and so the origin of this NLO force is simply that particle 1 is not quite moving on a straight line. Thus, to find the correction to the force, we must first determine the motion of particle 1 with NLO accuracy.

We obtain the first perturbative correction to the velocity and to the trajectory of particle 1 by integrating the leading order force, equation (6.12) from time $\tau_{1}=-\infty$ to a finite time $\tau_{1}$. This integral must converge as $\tau_{1} \rightarrow-\infty$, so we follow standard practice (see, for example, Jackson [138] p. 676) and replace $\bar{q} \cdot u_{1}$ in the argument of the exponential 
with $\bar{q} \cdot u_{1}+i \epsilon$. The correction to the velocity is then

$$
\begin{aligned}
m_{1} \Delta^{(1)} v_{1}^{\mu} & =i e^{2} Q_{1} Q_{2} \int \hat{d}^{4} \bar{q} \hat{\delta}\left(\bar{q} \cdot u_{2}\right) e^{-i \bar{q} \cdot b} \frac{\bar{q}^{\mu} u_{1} \cdot u_{2}-u_{2}^{\mu} \bar{q} \cdot u_{1}}{\bar{q}^{2}} \int_{-\infty}^{\tau_{1}} d \tau_{1} e^{-i\left(\bar{q} \cdot u_{1}+i \epsilon\right) \tau_{1}} \\
& =-e^{2} Q_{1} Q_{2} \int \hat{d}^{4} \bar{q} \hat{\delta}\left(\bar{q} \cdot u_{2}\right) e^{-i \bar{q} \cdot\left(b+u_{1} \tau_{1}\right)} \frac{\bar{q}^{\mu} u_{1} \cdot u_{2}-u_{2}^{\mu} \bar{q} \cdot u_{1}}{\bar{q}^{2}\left(\bar{q} \cdot u_{1}+i \epsilon\right)}
\end{aligned}
$$

where on the second line, we have displayed the $i \epsilon$ convergence factor in the denominator explicitly while leaving it implicit in the argument of the exponential. The leading correction to the position of the particle is given by integrating once more, with the result:

$$
\Delta^{(1)} x_{1}^{\mu}\left(\tau_{1}\right)=-i \frac{e^{2} Q_{1} Q_{2}}{m_{1}} \int \hat{d}^{4} \bar{q} \hat{\delta}\left(\bar{q} \cdot u_{2}\right) e^{-i \bar{q} \cdot\left(b+u_{1} \tau_{1}\right)} \frac{\bar{q}^{\mu} u_{1} \cdot u_{2}-u_{2}^{\mu} \bar{q} \cdot u_{1}}{\bar{q}^{2}\left(\bar{q} \cdot u_{1}+i \epsilon\right)^{2}} .
$$

We have now collected the information we need to compute the NLO Lorentz force $\Delta^{(1)} f^{\mu}$, and therefore the NLO impulse. Recalling that the field strength involved in the force is given by equation (6.11), it is easy to see that the NLO force is

$$
\begin{aligned}
\Delta^{(1)} f^{\mu}= & i e^{2} Q_{1} Q_{2} \int \hat{d}^{4} \bar{\ell} \hat{\delta}\left(\bar{\ell} \cdot u_{2}\right) e^{-i \bar{\ell} \cdot\left(b+u_{1} \tau_{1}\right)} \frac{\bar{\ell}^{\mu} u_{2}^{\nu}-\bar{\ell}^{\nu} u_{2}^{\mu}}{\bar{\ell}^{2}} \\
& \times\left(\frac{d}{d \tau_{1}} \Delta^{(1)} x_{1 \nu}\left(\tau_{1}\right)-i \bar{\ell} \cdot \Delta^{(1)} x_{1}\left(\tau_{1}\right) u_{1 \nu}\right),
\end{aligned}
$$

where we relabelled the variable of integration $\bar{q} \rightarrow \bar{\ell}$ for later convenience. Using our knowledge of the corrected trajectory, we evaluate

$$
\begin{aligned}
\frac{d}{d \tau} \Delta^{(1)} x_{1 \nu}\left(\tau_{1}\right)-i \bar{\ell} \cdot \Delta^{(1)} x_{1}\left(\tau_{1}\right) u_{1 \nu}= & -\frac{e^{2} Q_{1} Q_{2}}{m_{1}} \int \hat{d}^{4} \bar{q}^{\prime} \hat{\delta}\left(\bar{q}^{\prime} \cdot u_{2}\right) e^{-i \bar{q}^{\prime} \cdot\left(b+u_{1} \tau_{1}\right)} \\
& \times\left(\frac{\bar{q}_{\nu}^{\prime} u_{1} \cdot u_{2}-u_{2 \nu} \bar{q}^{\prime} \cdot u_{1}}{\bar{q}^{\prime 2}\left(\bar{q}^{\prime} \cdot u_{1}+i \epsilon\right)}+\frac{\bar{\ell} \cdot \bar{q}^{\prime} u_{1} \cdot u_{2} u_{1 \nu}}{\bar{q}^{\prime 2}\left(\bar{q}^{\prime} \cdot u_{1}+i \epsilon\right)^{2}}\right),
\end{aligned}
$$

omitting terms which vanish on the support of the delta functions in equation (6.17). The integral $\int_{-\infty}^{\infty} d \tau_{1} \Delta^{(1)} f^{\mu}$ is the next-to-leading order impulse, given explicitly by

$$
\begin{aligned}
\left.\Delta p_{1}^{\mu,(1)}\right|_{m_{2} \rightarrow \infty}= & \frac{i e^{4} Q_{1}^{2} Q_{2}^{2}}{m_{1}} \int \hat{d}^{4} \bar{q} \hat{\delta}\left(\bar{q} \cdot u_{1}\right) \hat{\delta}\left(\bar{q} \cdot u_{2}\right) e^{-i \bar{q} \cdot b} \int \hat{d}^{4} \ell \frac{\hat{\delta}\left(\bar{\ell} \cdot u_{2}\right)}{\bar{\ell}^{2}(\bar{\ell}-\bar{q})^{2}} \\
& \times\left[1+\frac{\bar{\ell} \cdot(\bar{\ell}-\bar{q})\left(u_{1} \cdot u_{2}\right)^{2}}{\left(\bar{\ell} \cdot u_{1}-i \epsilon\right)^{2}}\right] \bar{\ell}^{\mu} .
\end{aligned}
$$

To obtain this result, we shifted one of the variables of integration by setting $\bar{q}=\bar{q}^{\prime}+\bar{\ell}$.

This expression for the NLO impulse is not quite in the form we obtained using quantum methods in section 5.2. The necessary rearrangement is as follows. We exploit the change of variable $\bar{\ell}^{\prime}=\bar{q}-\bar{\ell}$ and define a vector integral

$$
I^{\mu}=\int \hat{d}^{4} \bar{\ell} \frac{\hat{\delta}\left(\bar{\ell} \cdot u_{2}\right)}{\bar{\ell}^{2}(\bar{\ell}-\bar{q})^{2}}\left[1+\frac{\bar{\ell} \cdot(\bar{\ell}-\bar{q})\left(u_{1} \cdot u_{2}\right)^{2}}{\left(\bar{\ell} \cdot u_{1}-i \epsilon\right)^{2}}\right] \bar{\ell}^{\mu}
$$


then we find

$$
\begin{aligned}
I^{\mu}= & \int \hat{d}^{4} \bar{\ell}^{\prime} \frac{\hat{\delta}\left(\overline{\ell^{\prime}} \cdot u_{2}\right)}{{\overline{\ell^{\prime}}}^{2}\left(\overline{\ell^{\prime}}-\bar{q}\right)^{2}}\left[1+\frac{\bar{\ell}^{\prime} \cdot\left(\overline{\ell^{\prime}}-\bar{q}\right)\left(u_{1} \cdot u_{2}\right)^{2}}{\left(\bar{\ell}^{\prime} \cdot u_{1}+i \epsilon\right)^{2}}\right]\left(\bar{q}^{\mu}-\bar{\ell}^{\prime \mu}\right) \\
= & \frac{1}{2} \int \hat{d}^{4} \overline{\bar{\ell}} \frac{\hat{\delta}\left(\bar{\ell} \cdot u_{2}\right)}{\bar{\ell}^{2}(\bar{\ell}-q)^{2}}\left\{\left[1+\frac{\bar{\ell} \cdot(\bar{\ell}-\bar{q})\left(u_{1} \cdot u_{2}\right)^{2}}{\left(\bar{\ell} \cdot u_{1}+i \epsilon\right)^{2}}\right] \bar{q}^{\mu}\right. \\
& \left.-i\left[\bar{\ell} \cdot(\bar{\ell}-\bar{q})\left(u_{1} \cdot u_{2}\right)^{2} \hat{\delta}^{\prime}\left(\bar{\ell} \cdot u_{1}\right)\right] \bar{\ell}^{\mu}\right\} .
\end{aligned}
$$

Putting the pieces together, we arrive at our final result for the NLO impulse when $m_{2} \rightarrow \infty$ :

$$
\begin{aligned}
\left.\Delta p_{1}^{\mu,(1)}\right|_{m_{2} \rightarrow \infty}= & \int \hat{d}^{4} \bar{q} \hat{\delta}\left(\bar{q} \cdot u_{1}\right) \hat{\delta}\left(\bar{q} \cdot u_{2}\right) e^{-i \bar{q} \cdot b} \frac{e^{4} Q_{1}^{2} Q_{2}^{2}}{2 m_{1}} \int \hat{d}^{4} \bar{\ell} \frac{\hat{\delta}\left(\bar{\ell} \cdot u_{2}\right)}{\overline{\ell^{2}(\bar{\ell}-\bar{q})^{2}}} \\
& \times\left[i \bar{q}^{\mu}\left(1+\frac{\bar{\ell} \cdot(\bar{\ell}-\bar{q})\left(u_{1} \cdot u_{2}\right)^{2}}{\left(\bar{\ell} \cdot u_{1}+i \epsilon\right)^{2}}\right)+\bar{\ell}^{\mu} \bar{\ell} \cdot(\bar{\ell}-\bar{q})\left(u_{1} \cdot u_{2}\right)^{2} \hat{\delta}^{\prime}\left(\bar{\ell} \cdot u_{1}\right)\right] .
\end{aligned}
$$

We have thereby reproduced all terms in eq. (5.38) which survive in the limit $m_{2} \rightarrow \infty$, consistent with our present assumption that $m_{2}$ is very large. The full result for the NLO impulse requires accounting for corrections to the field strength of particle 2 due to its motion. The mechanics of the calculation are very much the same as in the discussion above. Taking these additional effects into account, one finds that the NLO impulse is precisely eq. (5.38).

\subsection{Classical radiated momentum}

Our next topic is the momentum radiated during a classical collision, using the standard methods of classical field theory. We first discuss a general expression for the momentum radiation which is analogous to the all-order radiation formula, equation (4.39), we found using quantum methods. We then apply the formula at leading order, making explicit contact with the LO radiation kernel we computed in section 5.3.

\subsubsection{General expressions}

The electromagnetic stress-energy tensor,

$$
T^{\mu \nu}(x)=F^{\mu \alpha}(x) F_{\alpha}{ }^{\nu}(x)+\frac{1}{4} \eta^{\mu \nu} F^{\alpha \beta}(x) F_{\alpha \beta}(x),
$$

is the key quantity which describes the distribution and flux of energy and momentum. In particular, the (four-)momentum flux through a three dimensional surface $\partial \Omega$ with surface element $d \Sigma_{\nu}$ is,

$$
K^{\mu}=\int_{\partial \Omega} d \Sigma_{\nu} T^{\mu \nu}(x) .
$$

We are interested in the total momentum radiated as two particles scatter. At each time $t$, we therefore surround the two particles with a large sphere. The instantaneous flux of momentum is measured by integrating over the surface area of the sphere; the total momentum 
radiated is then the integral of this instantaneous flux over all times. It is straightforward to determine the momentum radiated by direct integration over these spheres using textbook methods, as discussed in appendix D.

A simpler but more indirect method is the following. We wish to use the Gauss theorem to write,

$$
K^{\mu}=\int_{\partial \Omega} d \Sigma_{\nu} T^{\mu \nu}(x)=\int d^{4} x \partial_{\nu} T^{\mu \nu}(x) .
$$

However, the spheres surrounding our particle are not the boundary of all spacetime: they do not include the timelike future and past boundaries. To remedy this, we use a trick due to Dirac [109].

The radiation we have in mind is causal, so we solve the Maxwell equation with retarded boundary conditions. We denote these fields by $F_{\text {ret }}^{\mu \nu}(x)$. We could equivalently solve the Maxwell equation using the advanced Green's function. If we wish to determine precisely the same fields $F_{\text {ret }}^{\mu \nu}(x)$ but using the advanced Green's function, we must add a homogeneous solution of the Maxwell equation. Fitting the boundary conditions in this way requires subtracting the incoming radiation field $F_{\text {in }}^{\mu \nu}(x)$ which is present in the advanced solution (but not in the retarded solution) and adding the outgoing radiation field (which is present in the retarded solution, but not the advanced solution.) In other words,

$$
F_{\text {ret }}^{\mu \nu}(x)-F_{\text {adv }}^{\mu \nu}(x)=-F_{\text {in }}^{\mu \nu}(x)+F_{\text {out }}^{\mu \nu}(x) .
$$

Now, the radiated momentum $K^{\mu}$ in which we are interested is described by $F_{\text {out }}^{\mu \nu}(x)$. The field $F_{\text {in }}^{\mu \nu}(x)$ transports the same total amount of momentum in from infinity, ie it transports momentum $-K^{\mu}$ out. Therefore the difference between the momenta transported out to infinity by the retarded and by the advanced fields is simply $2 K^{\mu}$. This is useful, because the contributions of the point-particle sources cancel in this difference.

The relationship between the momentum transported by the retarded and advanced field is reflected at the level of the Green's functions themselves. The difference in the Green's function takes an instructive form:

$$
\begin{aligned}
\tilde{G}_{\text {ret }}(\bar{k})-\tilde{G}_{\text {adv }}(\bar{k}) & =\frac{(-1)}{\left(\bar{k}^{0}+i \epsilon\right)^{2}-\overline{\boldsymbol{k}}^{2}}-\frac{(-1)}{\left(\bar{k}^{0}-i \epsilon\right)^{2}-\overline{\boldsymbol{k}}^{2}} \\
& =i\left(\Theta\left(\bar{k}^{0}\right)-\Theta\left(-\bar{k}^{0}\right)\right) \hat{\delta}\left(\bar{k}^{2}\right) .
\end{aligned}
$$

In this equation, $\overline{\boldsymbol{k}}$ denotes the spatial components of wavenumber four-vector $\bar{k}$. This difference is a homogeneous solution of the wave equation since it is supported on $\bar{k}^{2}=0$. The two terms correspond to positive and negative angular frequencies. As we will see, the relative sign ensures that the momenta transported to infinity add.

With this in mind, we return to the problem of computing the momentum radiated and write,

$$
2 K^{\mu}=\int_{\partial \Omega} d \Sigma_{\nu}\left(T_{\text {ret }}^{\mu \nu}(x)-T_{\text {adv }}^{\mu \nu}(x)\right) .
$$

In this difference, the contribution of the sources at timelike infinity cancel, so we may regard the surface $\partial \Omega$ as the boundary of spacetime. Therefore,

$$
2 K^{\mu}=\int d^{4} x \partial_{\nu}\left(T_{\mathrm{ret}}^{\mu \nu}(x)-T_{\mathrm{adv}}^{\mu \nu}(x)\right)=-\int d^{4} x\left(F_{\mathrm{ret}}^{\mu \nu}(x)-F_{\mathrm{adv}}^{\mu \nu}(x)\right) J_{\nu}(x),
$$


where the last equality follows from the equations of motion. We now pass to momentum space, noting that,

$$
F^{\mu \nu}(x)=-i \int \hat{d}^{4} \bar{k}\left(\bar{k}^{\mu} \tilde{A}^{\nu}(\bar{k})-\bar{k}^{\nu} \tilde{A}^{\mu}(\bar{k})\right) e^{-i \bar{k} \cdot x} .
$$

Using conservation of momentum, the radiated momentum becomes,

$$
\begin{aligned}
2 K^{\mu} & =i \int \hat{d}^{4} \bar{k} \bar{k}^{\mu}\left(\tilde{A}_{\text {ret }}^{\nu}(\bar{k})-\tilde{A}_{\text {adv }}^{\nu}(\bar{k})\right) \tilde{J}_{\nu}^{*}(\bar{k}), \\
& =-\int \hat{d}^{4} \bar{k} \bar{k}^{\mu}\left(\Theta\left(\bar{k}^{0}\right)-\Theta\left(-\bar{k}^{0}\right)\right) \hat{\delta}\left(\bar{k}^{2}\right) \tilde{J}^{\nu}(\bar{k}) \tilde{J}_{\nu}^{*}(\bar{k}) .
\end{aligned}
$$

The two different $\Theta$ functions arise from the outgoing and incoming radiation fields. Setting $k^{\prime \mu}=-k^{\mu}$ in the second term, and then dropping the prime, it is easy to see that the two terms add as anticipated. We arrive at a simple general result for the momentum radiated:

$$
\begin{aligned}
K^{\mu} & =-\int \hat{d}^{4} \bar{k} \Theta\left(\bar{k}^{0}\right) \hat{\delta}\left(\bar{k}^{2}\right) \bar{k}^{\mu} \tilde{J}^{\nu}(\bar{k}) \tilde{J}_{\nu}^{*}(\bar{k}) \\
& =-\int d \Phi(\bar{k}) \bar{k}^{\mu} \tilde{J}^{\nu}(\bar{k}) \tilde{J}_{\nu}^{*}(\bar{k}) .
\end{aligned}
$$

It is worth pausing to compare this general classical formula for the radiated momentum to the expression, eq. (4.41) which we derived in section 4.4. Evidently the radiation kernel we defined in eq. (4.40) is related to the classical current $\tilde{J}^{\mu}(\bar{k})$. This fact was anticipated in ref. [137]. Indeed, if we introduce a basis of polarisation vectors $\varepsilon^{\mu(h)}(\bar{k})$ associated with the wavevector $\bar{k}$ with helicity $h$, we may write the classical momentum radiated as,

$$
K^{\mu}=\sum_{h} \int d \Phi(\bar{k}) \bar{k}^{\mu}\left|\varepsilon^{(h)} \cdot \tilde{J}(\bar{k})\right|^{2},
$$

where here we have written the sum over helicities explicitly. In the next subsection, we will take this observation a step further by demonstrating that the leading order radiation kernel is proportional to $\varepsilon^{(h)} . \tilde{J}(\bar{k})$ on the support of the phase space integral in eq. (6.33).

\subsubsection{Application at leading order}

We have established a convenient, general formula for the momentum radiated in the classical theory. It is now rather straightforward to use this expression, as well as our perturbative knowledge of the trajectories, in order to determine the momentum radiated order by order in perturbation theory. We will again simplify our classical discussion by assuming that $m_{2}$ is so large that we can treat particle 2 as static. Our goal in this section will be to compare the classical momentum radiated with our previous quantum calculation for the expectation of this quantity, in the limit where this expectation is very sharply peaked. As we have seen, the general expressions for the radiation have the same structure so it will be enough for us to compare the radiation kernel to the classical current.

Static particles do not radiate, so we can ignore the contribution of particle 2 while evaluating the momentum radiated. To see that this is indeed the case, note that the part of the current involving particle 2 is

$$
J_{2}^{\mu}=e Q_{2} \int d \tau_{2} u_{2}^{\mu} \delta^{(4)}\left(x-u_{2} \tau_{2}\right) \Rightarrow \tilde{J}_{2}(\bar{k})=e Q_{2} u_{2}^{\mu} \hat{\delta}\left(\bar{k} \cdot u_{2}\right)
$$


Working in the rest frame of particle 2 , the delta function in $\tilde{J}_{2}(\bar{k})$ forces $\bar{k}^{0}=0$. But on the other hand the integral over $\bar{k}$ in the radiated momentum, eq. (6.32), requires that $\bar{k}^{0}=|\overline{\boldsymbol{k}}|$. The only solution is $\bar{k}^{\mu}=0$, so the integral vanishes.

Therefore we may replace the current $\tilde{J}^{\mu}(\bar{k})$ in the radiated momentum with the contribution $\tilde{J}_{1}^{\mu}(\bar{k})$ from particle 1 . This current is,

$$
\begin{aligned}
\tilde{J}_{1}^{\mu}(\bar{k})= & e Q_{1} \int d \tau_{1} v_{1}^{\mu}\left(\tau_{1}\right) e^{i \bar{k} \cdot x_{1}\left(\tau_{1}\right)} \\
= & e Q_{1} u_{1}^{\mu} \hat{\delta}\left(\bar{k} \cdot u_{1}\right) e^{i \bar{k} \cdot b} \\
& +e Q_{1} \int d \tau_{1} e^{i \bar{k} \cdot\left(b+u_{1} \tau_{1}\right)}\left(\Delta^{(1)} v_{1}^{\mu}\left(\tau_{1}\right)+i u_{1}^{\mu} \bar{k} \cdot \Delta^{(1)} x_{1}\left(\tau_{1}\right)\right)+\mathcal{O}\left(e^{5}\right)
\end{aligned}
$$

We can ignore the leading-order static part of this current just as we ignored the static current of particle 2. The dynamical part involves the perturbative correction to the trajectory of particle 1 , which we computed in section 6.1 .2 in order to determine the NLO impulse on particle 1 . With the help of these results, we can write down the NLO current of particle 1. Comparison to the quantum calculation is facilitated by resolving this current onto a basis of polarisation vectors and using eq. (6.32) for the momentum radiated. We find,

$$
\begin{aligned}
\varepsilon^{(h)} \cdot \tilde{J}_{1}(\bar{k}) \rightarrow & \frac{e^{3} Q_{1}^{2} Q_{2}}{m_{1}} \int \hat{d}^{4} \bar{q}_{1} \hat{d}^{4} \bar{q}_{2} \hat{\delta}\left(\bar{q}_{1} \cdot u_{1}\right) \hat{\delta}\left(\bar{q}_{2} \cdot u_{2}\right) \hat{\delta}^{(4)}\left(\bar{k}-\bar{q}_{1}-\bar{q}_{2}\right) e^{i \bar{q}_{1} \cdot b} \frac{1}{\bar{q}_{2}^{2}} \\
& \times\left[u_{2} \cdot \varepsilon^{(h)}-\frac{u_{1} \cdot u_{2} \bar{q}_{2} \cdot \varepsilon^{(h)}}{\bar{k} \cdot u_{1}}-\frac{\bar{k} \cdot u_{2} u_{1} \cdot \varepsilon^{(h)}}{\bar{k} \cdot u_{1}}\right. \\
& \left.+\frac{\bar{k} \cdot \bar{q}_{2} u_{1} \cdot u_{2} u_{1} \cdot \varepsilon^{(h)}}{\left(\bar{k} \cdot u_{1}\right)^{2}}\right]+\mathcal{O}\left(e^{5}\right) .
\end{aligned}
$$

We are now ready to compare this classical result to equation (5.47) for the radiation kernel we encountered in the quantum case. The expressions are proportional, with proportionality constant $e^{3} \hbar^{2}$ (and an irrelevant sign). This factor is precisely provided by the definition of the radiated momentum in terms of the radiation kernel, eq. (4.43). Thus, we see once again that the quantum and classical calculations match - as they must in the classical limit.

The calculation we have described in this subsection is an Abelian version of the nonAbelian calculation of Goldberger and Ridgway [91], which was motivated by the doublecopy relation between gauge theory and gravity. The fact that the classical current can be reproduced by taking a limit of a scattering amplitude was pointed out in ref. [137]. Recently, the classical computation was pushed to one higher order in perturbation theory by Shen [95]. It would be interesting to reproduce Shen's result using the methods of scattering amplitudes.

\subsection{Momentum conservation and the radiation reaction force}

Finally we turn to conservation of momentum in the classical theory. This is a celebrated problem in classical field theory, where the point particle approximation leads to wellknown issues. Problems arise because of the singular nature of the point particle source. 
In particular, the electromagnetic field at the position of a point charge is infinite, so to make sense of the Lorentz force acting on the particle the traditional route is to subtract the particle's own field from the full electromagnetic field in the force law. The result is a well-defined force, but conservation of momentum is lost.

Conservation of momentum is restored by including another force, the AbrahamLorentz-Dirac (ALD) force [105-109], acting on the particles. This gives rise to an impulse on particle 1 in addition to the impulse due to the Lorentz force; the change in the momentum due to the ALD force balances the momentum lost to radiation. The ALD impulse is,

$$
\Delta p_{1}^{\mu}=\frac{e^{2} Q_{1}^{2}}{6 \pi m_{1}} \int_{-\infty}^{\infty} d \tau\left(\frac{d^{2} p_{1}^{\mu}}{d \tau^{2}}+\frac{p_{1}^{\mu}}{m_{1}^{2}} \frac{d p_{1}}{d \tau} \cdot \frac{d p_{1}}{d \tau}\right) .
$$

To keep our discussion as simple as possible, we again take particle 2 to be static in this section.

Working in perturbation theory, the lowest order contribution to $d p_{1} / d \tau$ is of order $e^{2}$, due to the LO Lorentz force. Therefore $\Delta p_{1}^{\mu}$ is at least of order $e^{4}$. However, this potential contribution to the ALD impulse vanishes. To see this, recall that the LO force on particle 1 is given by eq. (6.12). The acceleration due to this leading order Lorentz force in turn gives rise to an ALD impulse of,

$$
\Delta p_{1}^{\mu}=\frac{e^{4} Q_{1}^{3} Q_{2}}{6 \pi m_{1}} \int \hat{d}^{4} \bar{q} \hat{\delta}\left(\bar{q} \cdot u_{1}\right) \hat{\delta}\left(\bar{q} \cdot u_{2}\right) e^{-i \bar{q} \cdot b} \bar{q} \cdot u_{1} \frac{\bar{q}^{\mu} u_{1} \cdot u_{2}-u_{2}^{\mu} \bar{q} \cdot u_{1}}{\bar{q}^{2}}=0 .
$$

An alternative point of view on the same result is to perform the time integral in equation (6.37), noting that the second term in the ALD force is higher order. The impulse is then proportional to $f^{\mu}(+\infty)-f^{\mu}(-\infty)$, the difference in the asymptotic Lorentz forces on particle 1. But at asymptotically large times the two particles are infinitely far away, so the Lorentz forces must vanish. Since this second argument does not rely on perturbation theory we may ignore the first term in the ALD force law in the remainder of the section.

Thus, the first non-vanishing impulse due to radiation reaction is of order $e^{6}$. Since we only need the leading order Lorentz force, eq. (6.12), to evaluate the ALD impulse, we can anticipate that the result will be very simple. Indeed, integrating the ALD force, we find that the impulse on particle 1 due to radiation reaction is

$$
\begin{aligned}
\Delta p_{1}^{\mu}= & \frac{e^{6} Q_{1}^{4} Q_{2}^{2}}{6 \pi m_{1}^{2}} u_{1}^{\mu} \int \hat{d}^{4} \bar{q} \hat{d}^{4} \bar{q}^{\prime} \hat{\delta}\left(\bar{q} \cdot u_{2}\right) \hat{\delta}\left(\bar{q}^{\prime} \cdot u_{2}\right) \hat{\delta}\left(u_{1} \cdot\left(\bar{q}-\bar{q}^{\prime}\right)\right) e^{-i b \cdot\left(\bar{q}-\bar{q}^{\prime}\right)} \frac{1}{\bar{q}^{2} \bar{q}^{\prime 2}} \\
& \times\left[\left(\bar{q} \cdot u_{1}\right)^{2}+\bar{q} \cdot \bar{q}^{\prime}\left(u_{1} \cdot u_{2}\right)^{2}\right] .
\end{aligned}
$$

This is precisely the expression (5.55) we found using our quantum mechanical approach in section 5.4. In that case, the simple final result arose after integrating the square of a more complicated five-point tree amplitude over the phase space of the photon. 


\section{Discussion and conclusions}

In order to apply on-shell scattering amplitudes to the calculation of classically observable quantities, one needs a definition of the observables in the quantum theory. One also needs a set of rules and a path for taking the classical limit of the quantum observables. Ideally the rules will be straightforward, simpler to apply than the full quantum calculation, and the path will lead gently downhill.

In this article, we have shown how to construct suitable expressions for two example observables, the momentum transfer or impulse (3.18) on a particle and the momentum emitted as radiation (3.34) during the scattering of two spinless point particles. We have shown how to restore $\hbar$ s and classify momenta in section 2 ; in section 4 , how to choose suitable wavefunctions for the incoming particles and under what conditions the classical limit is simple. In section 4 , we further gave simplified leading- and next-to-leadingorder expressions in terms of on-shell scattering amplitudes for the impulse in eqs. (4.30) and (4.33), and for the radiated momentum in eq. (4.41). These expressions apply directly to both electrodynamics and gravity. In section 5, we used explicit expressions for the amplitudes in quantum electrodynamics to obtain results in the classical theory. We have been careful throughout to ensure that our methods correctly incorporate conservation of momentum, without the need to introduce an analogue of the Abrahams-Lorentz-Dirac radiation reaction.

Other observables should be readily accessible by similar derivations: the same two observables, but for the scattering of spinning particles; the change in spin during scattering; the polarization of emitted radiation; the radiation flux as a function of spherical angle; and more. Higher-order corrections, to the extent they are unambiguously defined in the classical theory, require the harder work of computing two- and higher-loop amplitudes, but the formalism of this article will continue to apply.

Our setup has features in common with two related, but somewhat separate, areas of current interest. One area is the study of the potential between two massive bodies [69-78, 80-82]. The second is the study of particle scattering in the eikonal regime [118-135]. Diagrammatically, the study of the potential is evidently closely related to the impulse of the present article. To some extent this is by design: we wished to construct an on-shell observable related to the potential. But we have also been able to construct an additional observable, the radiated momentum, which is related to the gravitational flux.

It is interesting that classical physics emerges in the study of the high-energy limit of quantum scattering [118-121], see also refs. [84, 85]. Indeed the classical center-ofmomentum scattering angle can be obtained from the eikonal function (see, for example ref. [127]). This latter function must therefore be related as well to the impulse, even though we have not taken any high-energy limit. Indeed, the impulse and the scattering angle are equivalent at $\mathrm{LO}$ and NLO, because no momentum is radiated at these orders. Therefore the scattering angle completely determines the change in momentum of the particles (and vice versa). The connection to the eikonal function should be interesting to explore. 
At NNLO, on the other hand, the equivalence between the angle and the impulse fails. This is because of radiation: knowledge of the angle tells you where the particles went, but not how fast. In this respect the impulse is more informative than the angle. Eikonal methods are still applicable in the radiative case [122], so they should reproduce the high-energy limit of the expectation value of the radiated momentum. Meanwhile at low energies, methods based on soft theorems could provide a bridge between the impulse and the radiated momentum [102-104].

The NLO scattering angle is, in fact, somewhat simpler than the impulse: see ref. [134] for example. Thanks to the exponentiation at play in the eikonal limit, it is the triangle diagram which is responsible for the NLO correction. But the impulse contains additional contributions, as we discussed in section 5.2. Perhaps this is because the impulse must satisfy an on-shell constraint, unlike the angle.

We restricted attention to spinless scattering in this article. In this context, the impulse (or equivalently, the angle) is the only physical observable at LO and NLO, and completely determines the interaction Hamiltonian between the two particles [84, 85]. The situation is richer in the case of arbitrarily aligned spins: then the change in spins of the particles is an observable which is not determined by the scattering angle. We expect that this observable can also be extracted from scattering amplitudes using our methods.

As in any application of traditional scattering amplitudes, however, time-dependent phenomena are not readily accessible. This reflects the fact that amplitudes are the matrix elements of a time evolution operator from the far past to the far future. For a direct application of our methods to the time-dependent gravitational waveform, we must overcome this limitation. One possible path for future investigation would start from the fact that the two observables we have discussed are essentially expectation values. They are therefore most naturally discussed using the time-dependent in-in formalism, which has a well-known Schwinger-Keldysh diagrammatic formulation. Whether the double copy applies in this context, offering an avenue to simpler calculations in gravity, remains to be explored.

An advantage of our methods is that they naturally incorporate the actual radiated flux, which is obviously a key physical quantity for gravitational wave observatories. The formalism presented in this paper opens the door to applying the many tools and recent advances in the study of scattering amplitudes to computing a variety of observables at higher orders in massless classical field theories. The application of one of these insights, the double copy, to observables in gravity should prove particularly fruitful.

\section{Acknowledgments}

We thank Richard Ball, Arjun Berera, Zvi Bern, Lucile Cangemi, John Joseph Carrasco, Clifford Cheung, Maria Derda, Einan Gardi, Grisha Korchemsky, Andrés Luna, Rafael Porto, Justin Vines, and Chris White for helpful discussions and comments. DAK also thanks the Galileo Galilei Institute where this work was completed during the 2018 Amplitudes in the LHC era program. This work was supported in part by the French Agence Nationale pour la Recherche, under grant ANR-17-CE31-0001-01. BM is supported by 
STFC studentship ST/R504737/1. DOC is an IPPP associate, and thanks the IPPP for on-going support as well as for hospitality during this work. He is supported in part by the Marie Curie FP7 grant 631370 and by the STFC consolidated grant Particle Physics at the Higgs Centre. Several of our figures were produced with the help of the TikZ-Feynman package [139].

\section{A Conventions}

We have chosen a mostly-minus signature metric. To deal with a proliferation of factors of $2 \pi$ appearing in measures, we use a short-hand notation first defined in equations $(2.3)$ and $(3.2)$

$$
\begin{aligned}
\hat{\delta}^{(n)}(u) & \equiv(2 \pi)^{n} \delta^{(n)}(u)=\int d^{n} x e^{i u \cdot x}, \\
\hat{d}^{n} q & \equiv \frac{d^{n} q}{(2 \pi)^{n}} .
\end{aligned}
$$

The measure for integrals over Lorentz-invariant phase space, eq. (3.4), is

$$
d \Phi\left(k_{i}\right) \equiv \hat{d}^{4} k_{i} \hat{\delta}^{(+)}\left(k_{i}^{2}-m_{i}^{2}\right) .
$$

We occasionally find it convenient to separate a Lorentz vector $x^{\mu}$ into its time component $x^{0}$ and its three spatial components $\boldsymbol{x}$ so that $x^{\mu}=\left(x^{0}, x^{i}\right)=\left(x^{0}, \boldsymbol{x}\right)$ (where $i=1,2,3$.)

Our convention for Fourier transforms, eq. (6.9), is

$$
\begin{aligned}
& f(x)=\int \hat{d}^{4} \bar{q} \tilde{f}(\bar{q}) e^{-i \bar{q} \cdot x}, \\
& \tilde{f}(\bar{q})=\int d^{4} x f(x) e^{i \bar{q} \cdot x} .
\end{aligned}
$$

\section{B Linear wavefunction integrals}

In order to compute the wavefunction normalization in eq. (4.18), we must compute the following integral,

$$
m^{-2} \int d \Phi(p) \exp \left[-\frac{2 p \cdot u}{m \xi}\right]
$$

Let us introduce the following parametrization for the on-shell phase space,

$$
p^{\mu}=E(\cosh \zeta, \sinh \zeta \sin \theta \cos \phi, \sinh \zeta \sin \theta \sin \phi, \sinh \zeta \cos \theta)
$$

so that,

$$
\begin{aligned}
d \Phi(p) & =(2 \pi)^{-3} \hat{d} E d \zeta d \Omega_{2} \hat{\delta}\left(E^{2}-m^{2}\right) \Theta(E) E^{3} \sinh ^{2} \zeta \\
& =(2 \pi)^{-3} \hat{d} E d \zeta d \theta d \phi \hat{\delta}\left(E^{2}-m^{2}\right) \Theta(E) E^{3} \sinh ^{2} \zeta \sin \theta,
\end{aligned}
$$

with $\zeta$ running over $[0, \infty], \theta$ over $[0, \pi]$, and $\phi$ over $[0,2 \pi]$. Performing the $E$ integration, we obtain,

$$
d \Phi(p) \rightarrow \frac{m^{2}}{2(2 \pi)^{3}} d \zeta d \theta d \phi \sinh ^{2} \zeta \sin \theta
$$


along with $E=m$ in the integrand. The integral must be a Lorentz-invariant function of $u$; as the only available Lorentz invariant is $u^{2}=1$, we conclude that the result must be a function of $\xi$ alone. We can compute it in the rest frame of $u$, where our desired integral is,

$$
\frac{1}{2(2 \pi)^{3}} \int_{0}^{\infty} d \zeta \sinh ^{2} \zeta \int_{0}^{\pi} d \theta \sin \theta \int_{0}^{2 \pi} d \phi \exp \left[-2 \frac{\cosh \zeta}{\xi}\right]=\frac{1}{2(2 \pi)^{2}} \xi K_{1}(2 / \xi),
$$

where $K_{1}$ is a modified Bessel function of the second kind. The normalization condition (3.12) then yields,

$$
\frac{2 \sqrt{2} \pi}{\xi^{1 / 2} K_{1}^{1 / 2}(2 / \xi)}
$$

for the wavefunction's normalization.

Next, we compute $\left\langle p^{\mu}\right\rangle$. Lorentz invariance implies that the expectation value must be proportional to $u^{\mu}$; again computing in the rest frame, we find that,

$$
\left\langle p^{\mu}\right\rangle=m u^{\mu} \frac{K_{2}(2 / \xi)}{K_{1}(2 / \xi)} .
$$

The phase-space measure fixes $\left\langle p^{2}\right\rangle=m^{2}$, so we conclude that,

$$
\begin{aligned}
\frac{\left\langle(\Delta p)^{2}\right\rangle}{\left\langle p^{2}\right\rangle} & =1-\frac{\langle p\rangle^{2}}{\left\langle p^{2}\right\rangle} \\
& =1-\frac{K_{2}^{2}(2 / \xi)}{K_{1}^{2}(2 / \xi)} \\
& =-\frac{3}{2} \xi+\mathcal{O}\left(\xi^{2}\right) .
\end{aligned}
$$

We must further evaluate an integral with an on-shell delta function,

$$
T(\bar{q})=\frac{\mathcal{N}^{2}}{\hbar m^{3}} \exp \left[-\frac{\hbar \bar{q} \cdot u}{m \xi}\right] \int d \Phi(p) \hat{\delta}\left(2 p \cdot \bar{q} / m+\hbar \bar{q}^{2} / m\right) \exp \left[-\frac{2 p \cdot u}{m \xi}\right] .
$$

This integral is dimensionless, and can depend only on two Lorentz invariants, $\bar{q} \cdot u$ and $\bar{q}^{2}$, along with $\xi$. It is convenient to write it as a function of two dimensionless variables built out of these invariants,

$$
\begin{aligned}
\omega & \equiv \frac{\bar{q} \cdot u}{\sqrt{-\bar{q}^{2}}}, \\
\tau & \equiv \frac{\hbar \sqrt{-\bar{q}^{2}}}{2 m} .
\end{aligned}
$$

We again work in the rest frame of $u^{\mu}$, and without loss of generality, choose the $z$-axis of the $p$ integration to lie along the direction of $\overline{\boldsymbol{q}}$. The only components that appear in the integral are then $\bar{q}^{0}$ and $\bar{q}^{z}$; after integration, we can obtain the dependence on $\tau$ and $\omega$ via the replacements,

$$
\begin{aligned}
& \bar{q}^{0} \rightarrow \frac{2 m \omega \tau}{\hbar} \\
& \bar{q}^{z} \rightarrow \frac{2 m \sqrt{1+\omega^{2}} \tau}{\hbar} ;
\end{aligned}
$$


and hence,

$$
-\bar{q}^{2} \rightarrow \frac{4 m^{2} \tau^{2}}{\hbar^{2}}
$$

Using the measure (B.4), we find,

$$
\begin{aligned}
& \frac{1}{2 \pi \hbar m \xi K_{1}(2 / \xi)} \exp \left[-\frac{\hbar \bar{q}^{0}}{m \xi}\right] \\
& \quad \times \int_{0}^{\infty} d \zeta \sinh ^{2} \zeta \int_{0}^{\pi} d \theta \sin \theta \int_{0}^{2 \pi} d \phi \exp \left[-2 \frac{\cosh \zeta}{\xi}\right] \\
& \quad \times \hat{\delta}\left(2 \bar{q}^{0} \cosh \zeta-2 \bar{q}^{z} \sinh \zeta \cos \theta+\hbar \bar{q}^{2} / m\right) .
\end{aligned}
$$

The $\phi$ integral is trivial, and we can use the delta function to do the $\theta$ integral,

$$
\begin{aligned}
& \frac{1}{2 \hbar m \bar{q}^{z} \xi K_{1}(2 / \xi)} \exp \left[-\frac{\hbar \bar{q}^{0}}{m \xi}\right] \int_{0}^{\infty} d \zeta \sinh \zeta \exp \left[-2 \frac{\cosh \zeta}{\xi}\right] \\
& \quad \times \Theta\left(1+\bar{q}^{0} \operatorname{coth} \zeta / \bar{q}^{z}+\hbar \bar{q}^{2} \operatorname{csch} \zeta /\left(2 m \bar{q}^{z}\right)\right) \\
& \quad \times \Theta\left(1-\bar{q}^{0} \operatorname{coth} \zeta / \bar{q}^{z}-\hbar \bar{q}^{2} \operatorname{csch} \zeta /\left(2 m \bar{q}^{z}\right)\right) .
\end{aligned}
$$

In the $\hbar \rightarrow 0$ limit, the first theta function will have no effect, even with $\bar{q}^{2}<0$. Changing variables to $w=\cosh \zeta$, the second theta function will impose the constraint,

$$
w \geq \frac{\bar{q}^{z} \sqrt{1-\hbar^{2} \bar{q}^{2} /\left(4 m^{2}\right)}}{\sqrt{-\bar{q}^{2}}}-\frac{\hbar \bar{q}^{0}}{2 m} .
$$

In terms of $\omega$ and $\tau$, this constraint is,

$$
w \geq \sqrt{1+\omega^{2}} \sqrt{1+\tau^{2}}-\omega \tau .
$$

Up to corrections of $\mathcal{O}(\hbar)$, the right-hand side is greater than 1, and so becomes the lower limit of integration. The result for the integral is then,

$$
\begin{aligned}
T(\bar{q}) & =\frac{1}{8 m^{2} \sqrt{1+\omega^{2}} \tau K_{1}(2 / \xi)} \exp \left[-2 \frac{\omega \tau}{\xi}\right] \exp \left[-\frac{2}{\xi}\left(\sqrt{1+\omega^{2}} \sqrt{1+\tau^{2}}-\omega \tau\right)\right] \\
& =\frac{1}{4 \hbar m \sqrt{(\bar{q} \cdot u)^{2}-\bar{q}^{2}} K_{1}(2 / \xi)} \exp \left[-\frac{2}{\xi} \frac{\sqrt{(\bar{q} \cdot u)^{2}-\bar{q}^{2}}}{\sqrt{-\bar{q}^{2}}} \sqrt{1-\hbar^{2} \bar{q}^{2} /\left(4 m^{2}\right)}\right] .
\end{aligned}
$$

How does this function behave in the $\hbar, \xi \rightarrow 0$ limit (with $\bar{q}$ fixed)? The Bessel function simplifies,

$$
\frac{1}{K_{1}(2 / \xi)} \sim \frac{2}{\sqrt{\pi} \sqrt{\xi}} \exp \left[\frac{2}{\xi}\right]
$$

so we must take into account a modification of the exponent. In the limit, $\sqrt{\xi} \sim \hbar$, so that $\hbar \sqrt{\xi} \sim \xi$, and $T$ has the form shown in eq. (4.27),

$$
\frac{1}{\xi} \exp \left[-\frac{f(\bar{q})}{\xi}\right]
$$


which will yield a delta function so long as $f(\bar{q})$ is positive. To figure out its argument, recall that $\bar{q}^{2}<0$, and use a parametrization analogous that in eq. (B.2),

$$
\bar{q}^{\mu}=E_{\bar{q}}(\sinh \zeta, \cosh \zeta \sin \theta \cos \phi, \cosh \zeta \sin \theta \sin \phi, \cosh \zeta \cos \theta),
$$

so that again working in the rest frame of $u^{\mu}$, the exponent in eq. (B.17) (including the term from eq. (B.18)) is,

$$
-\frac{2}{\xi}\left(\cosh \zeta \sqrt{1+\hbar^{2} E_{\bar{q}}^{2} /\left(4 m^{2}\right)}-1\right)
$$

so that the delta function will ultimately localize

$$
\cosh \zeta \rightarrow \frac{1}{\sqrt{1+\hbar^{2} E_{\bar{q}}^{2} /\left(4 m^{2}\right)}}=1-\frac{\hbar^{2} E_{\bar{q}}^{2}}{8 m^{2}}+\mathcal{O}\left(\hbar^{4}\right),
$$

or translating back to Lorentz-invariant expressions, localize

$$
\bar{q} \cdot u+\frac{\hbar^{2}}{8 m^{2}} \bar{q} \cdot u \bar{q}^{2}
$$

to zero.

\section{Angular integrals}

In section 5.4 we encountered an integral over the on-shell phase space of a photon, namely eq. (5.53) which we reproduce here for ease of discussion:

$$
\begin{aligned}
Y_{\mathrm{r}}^{\mu}= & \int d \Phi(\bar{k}) \hat{\delta}\left(u_{1} \cdot \bar{k}-\bar{E}\right) \bar{k}^{\mu}\left[1+\frac{\left(u_{1} \cdot u_{2}\right)^{2}\left(\bar{q} \cdot \bar{q}^{\prime}\right)}{\bar{E}^{2}}+\frac{\left(u_{2} \cdot \bar{k}\right)^{2}}{\bar{E}^{2}}\right. \\
& \left.-\frac{\left(u_{1} \cdot u_{2}\right)\left(u_{2} \cdot \bar{k}\right) \bar{k} \cdot\left(\bar{q}+\bar{q}^{\prime}\right)}{\bar{E}^{3}}+\frac{\left(u_{1} \cdot u_{2}\right)^{2}(\bar{k} \cdot \bar{q})\left(\bar{k} \cdot \bar{q}^{\prime}\right)}{\bar{E}^{4}}\right] .
\end{aligned}
$$

In this appendix we will perform the integral over $\bar{k}$. We begin by defining

$$
\begin{aligned}
y_{0} & \equiv \int d \Phi(\bar{k}) \hat{\delta}\left(u_{1} \cdot \bar{k}-\bar{E}\right), \\
y_{1}^{\mu} & \equiv \int d \Phi(\bar{k}) \hat{\delta}\left(u_{1} \cdot \bar{k}-\bar{E}\right) \bar{k}^{\mu}, \\
y_{2}^{\mu \nu} & \equiv \int d \Phi(\bar{k}) \hat{\delta}\left(u_{1} \cdot \bar{k}-\bar{E}\right) \bar{k}^{\mu} \bar{k}^{\nu}, \\
y_{3}^{\mu \nu \rho} & \equiv \int d \Phi(\bar{k}) \hat{\delta}\left(u_{1} \cdot \bar{k}-\bar{E}\right) \bar{k}^{\mu} \bar{k}^{\nu} \bar{k}^{\rho} .
\end{aligned}
$$

We may then write

$$
\begin{aligned}
Y_{\mathrm{r}}^{\mu}= & \left(1+\frac{\left(u_{1} \cdot u_{2}\right)^{2}\left(\bar{q} \cdot \bar{q}^{\prime}\right)}{\bar{E}^{2}}\right) y_{1}^{\mu} \\
& +\left(\frac{u_{2 \nu} u_{2 \rho}}{\bar{E}^{2}}-\frac{\left(u_{1} \cdot u_{2}\right) u_{2 \nu}\left(\bar{q}_{\rho}+\bar{q}_{\rho}^{\prime}\right)}{\bar{E}^{3}}+\frac{\left.\left(u_{1} \cdot u_{2}\right)^{2} \bar{q}_{\nu} \bar{q}_{\rho}^{\prime}\right)}{\bar{E}^{4}}\right) y_{3}^{\mu \nu \rho} .
\end{aligned}
$$


Thus we need to know the integrals $y_{1}^{\mu}$ and $y_{3}^{\mu \nu \rho}$. It is also convenient to compute $y_{0}$, but we will omit the calculation of $y_{2}$.

We begin by computing $y_{0}$ : as we will see, this integral appears in the evaluation of the rest. It is convenient to work in the frame where particle 1 is stationary, so $u_{1}^{\mu}=(1, \mathbf{0})$. Then we can write

$$
y_{0}=\frac{1}{(2 \pi)^{2}} \int_{-\infty}^{\infty} d \bar{k}^{0} \int_{0}^{\infty} d \bar{\lambda} \bar{\lambda}^{2} \int d \Omega_{2} \Theta\left(\bar{k}^{0}\right) \delta\left(\bar{k}^{0}-\bar{E}\right) \delta\left(\left(\bar{k}^{0}\right)^{2}-\bar{\lambda}^{2}\right),
$$

where $\lambda=|\boldsymbol{k}|$. Each integral is straightforward. The integral over solid angle $d \Omega_{2}$ evaluates to $4 \pi$; we perform the $\bar{k}^{0}$ integral using the first delta function to discover a factor $\Theta(\bar{E})$, and finally we use the second delta function to perform the $\bar{k}$ integral. The result is

$$
y_{0}=\frac{\bar{E}}{2 \pi} \Theta(\bar{E}) \text {. }
$$

To evaluate $y_{1}^{\mu}$, we exploit Lorentz covariance which dictates that

$$
y_{1}^{\mu}=\int d \Phi(\bar{k}) \hat{\delta}\left(u_{1} \cdot \bar{k}-\bar{E}\right) \bar{k}^{\mu}=a_{1} u_{1}^{\mu},
$$

where $a_{1}$ is a scalar factor to be determined. In fact it is trivial to compute $a_{1}$ by dotting this expression into $u_{1}$; then the delta function enforces $a_{1}=y_{1} \cdot u_{1}=\bar{E} y_{0}$, so that

$$
y_{1}^{\mu}=\frac{\bar{E}^{2}}{2 \pi} u_{1}^{\mu} \Theta(\bar{E})
$$

In the same manner, Lorentz covariance and the symmetries of the integral require that,

$$
y_{3}^{\mu \nu \rho}=a_{3} u_{1}^{\mu} u_{1}^{\nu} u_{1}^{\rho}+b_{3}\left(u_{1}^{\mu} \eta^{\nu \rho}+u_{1}^{\nu} \eta^{\mu \rho}+u_{1}^{\rho} \eta^{\mu \nu}\right),
$$

in terms of scalar factors $a_{3}$ and $b_{3}$ which we must determine. To do so, we contract $y_{3}^{\mu \nu \rho}$ with $u_{1 \mu} u_{1 \nu} u_{1 \rho}$ and with $\eta_{\mu \nu} u_{1}^{\rho}$ to develop simultaneous equations for $a_{3}$ and $b_{3}$ :

$$
\begin{aligned}
u_{1 \mu} u_{1 \nu} u_{1 \rho} y_{3}^{\mu \nu \rho} & =a_{3}+3 b_{3}=\bar{E}^{3} y_{0}, \\
\eta_{\mu \nu} u_{1 \rho} y_{3}^{\mu \nu \rho} & =a_{3}+6 b_{3}=0 .
\end{aligned}
$$

As this system has the solution $a=\frac{\bar{E}^{4}}{\pi} \Theta(\bar{E})$ and $b=-\frac{\bar{E}^{4}}{6 \pi} \Theta(\bar{E})$, we learn that

$$
y_{3}^{\mu \nu \rho}=\frac{\bar{E}^{4}}{\pi}\left(u_{1}^{\mu} u_{1}^{\nu} u_{1}^{\rho}-\frac{1}{6}\left(u_{1}^{\mu} \eta^{\nu \rho}+u_{1}^{\nu} \eta^{\mu \rho}+u_{1}^{\rho} \eta^{\mu \nu}\right)\right) \Theta(\bar{E}) .
$$

\section{An alternative classical point of view on momentum radiation}

In the main text, we provided a classical derivation of a formula for the momentum radiated in electrodynamics. The result, eq. (6.32), was

$$
K^{\mu}=-\int d \Phi(\bar{k}) \bar{k}^{\mu} \tilde{J}^{\nu}(\bar{k}) \tilde{J}_{\nu}^{*}(\bar{k}) .
$$


The derivation maintained covariance, but involved a rather indirect extraction of the radiation field. In this appendix, we will derive the same formula from a somewhat simpler point of view, using textbook methods (see, for example, [140]).

We begin as in section 6.2.1 with the observation that the momentum radiated can be obtained by integrating the stress-energy tensor over the surfaces of large two-dimensional spheres, and over all time:

$$
K^{\mu}=\lim _{|\boldsymbol{x}| \rightarrow \infty} \int_{-\infty}^{\infty} d t \int d \Omega_{2}|\boldsymbol{x}|^{2} n_{\nu} T^{\mu \nu}(x)
$$

where $n \cdot e_{r}=1$ and $e_{r}$ is the unit radial outgoing vector on the spheres. To evaluate this integral, we need an expression for the gauge field $A^{\mu}(x)$. Working in Lorenz gauge, this can be obtained by solving the Maxwell equation using a Green's function $G(x)$ :

$$
A^{\mu}(x)=\int d^{4} x^{\prime} G\left(x-x^{\prime}\right) J^{\mu}\left(x^{\prime}\right) .
$$

In our situation, we assume that there is no incoming radiation, so the appropriate choice is the retarded Green's function $G_{\text {ret }}(x)$ which is explicitly

$$
G_{\text {ret }}(x)=\frac{1}{2 \pi} \Theta\left(x^{0}\right) \delta\left(x^{2}\right)=\frac{1}{4 \pi|\boldsymbol{x}|} \delta\left(x^{0}-|\boldsymbol{x}|\right),
$$

where $x^{\mu}=(t, \boldsymbol{x})$. As we will see, it is useful to Fourier transform $A^{\mu}(x)$ in the time dimension alone. We write,

$$
\begin{aligned}
A^{\mu}(\omega, \boldsymbol{x}) & \equiv \int d t e^{i \omega t} A^{\mu}(t, \boldsymbol{x}) \\
& =\int d t^{\prime} d^{3} x^{\prime} \frac{e^{i \omega t^{\prime}} e^{i \omega\left|\boldsymbol{x}-\boldsymbol{x}^{\prime}\right|}}{4 \pi\left|\boldsymbol{x}-\boldsymbol{x}^{\prime}\right|} J^{\mu}\left(t^{\prime}, \boldsymbol{x}^{\prime}\right) \\
& =\frac{1}{4 \pi} \int d^{3} x^{\prime} \frac{e^{i \omega\left|\boldsymbol{x}-\boldsymbol{x}^{\prime}\right|}}{\left|\boldsymbol{x}-\boldsymbol{x}^{\prime}\right|} J^{\mu}\left(\omega, \boldsymbol{x}^{\prime}\right) .
\end{aligned}
$$

This is an exact expression for the gauge field. However, we only need to know the gauge field on the surface of spheres surrounding our interacting particles, with radii very large compared to the separation of the particles. So we can expand the fields at large $|\boldsymbol{x}|$. In particular, the $\boldsymbol{x}^{\prime}$ integral in (D.5) extends only over the spatial support of the source $J^{\mu}\left(t^{\prime}, \boldsymbol{x}^{\prime}\right)$. We assume that radii of the spheres are always very large compared to this spatial size (which in our application will be of order $b$ ). We can therefore assume that $\left|\boldsymbol{x}^{\prime}\right| /|\boldsymbol{x}| \ll 1$.

Some care needs to be taken in the expansion of equation (D.5), however, since another length scale appears in the problem. This is the wavelength $\lambda$ of the radiation, of order $1 / \omega$. This wavelength need not be of order $b$, and in particular the quantity $\omega\left|\boldsymbol{x}^{\prime}\right| \sim\left|\boldsymbol{x}^{\prime}\right| / \lambda$ need not be small. We therefore expand the field as

$$
A^{\mu}(\omega, \boldsymbol{x}) \simeq \frac{e^{i \omega|\boldsymbol{x}|}}{4 \pi|\boldsymbol{x}|} \int d^{3} x^{\prime} e^{-i \omega \hat{\boldsymbol{x}} \cdot \boldsymbol{x}^{\prime}} J^{\mu}\left(\omega, \boldsymbol{x}^{\prime}\right)
$$


where $\hat{\boldsymbol{x}}=\boldsymbol{x} /|\boldsymbol{x}|$ is a unit three-dimensional vector. Defining the wavevector $\overline{\boldsymbol{k}}=\omega \hat{\boldsymbol{x}}$, can recognise the spatial Fourier transform present in eq. (D.6), and learn that

$$
A^{\mu}(\omega, \boldsymbol{x}) \simeq \frac{e^{i \omega|\boldsymbol{x}|}}{4 \pi|\boldsymbol{x}|} J^{\mu}(\omega, \overline{\boldsymbol{k}})=\frac{e^{i \omega|\boldsymbol{x}|}}{4 \pi|\boldsymbol{x}|} \tilde{J}^{\mu}(\bar{k}),
$$

where $\bar{k}^{\mu}=(\omega, \boldsymbol{k})$. Note that $\bar{k} \cdot \bar{k}=0$. For later use, we remark that

$$
A^{\mu}(-\omega, \boldsymbol{x})=\frac{e^{-i \omega|\boldsymbol{x}|}}{4 \pi|\boldsymbol{x}|} \tilde{J}^{\mu}(-\bar{k}) .
$$

It is straightforward to obtain the field strength $F^{\mu \nu}$ from our gauge field. Neglecting terms which are subdominant at large distances, we find that

$$
\begin{aligned}
F^{\mu \nu}(x) & \equiv \int \frac{d \omega}{2 \pi} e^{-i \omega t} F^{\mu \nu}(\omega, \boldsymbol{x}) \\
& =-i \int \frac{d \omega}{2 \pi} e^{-i \omega t}\left(\bar{k}^{\mu} A^{\nu}(\omega, \boldsymbol{x})-\bar{k}^{\nu} A^{\mu}(\omega, \boldsymbol{x})\right) .
\end{aligned}
$$

It is also worth noting that $\bar{k}_{\mu} A^{\mu}(\omega, \boldsymbol{x})=0$ as a consequence of current conservation.

We now return to the momentum radiated. We first trade the time integral of the stress-energy tensor for a frequency integral:

$$
\begin{aligned}
\int_{-\infty}^{\infty} d t & \left(F^{\mu \alpha}(x) F_{\alpha}{ }^{\nu}(x)+\frac{1}{4} \eta^{\mu \nu} F^{\alpha \beta}(x) F_{\alpha \beta}(x)\right) \\
= & \int \frac{d \omega}{2 \pi} \int_{-\infty}^{\infty} d t e^{-i \omega t}\left(F^{\mu \alpha}(\omega, \boldsymbol{x}) F_{\alpha}{ }^{\nu}(t, \boldsymbol{x})+\frac{1}{4} \eta^{\mu \nu} F^{\alpha \beta}(\omega, \boldsymbol{x}) F_{\alpha \beta}(t, \boldsymbol{x})\right) \\
& =\int \frac{d \omega}{2 \pi}\left(F^{\mu \alpha}(\omega, \boldsymbol{x}) F_{\alpha}{ }^{\nu}(-\omega, \boldsymbol{x})+\frac{1}{4} \eta^{\mu \nu} F^{\alpha \beta}(\omega, \boldsymbol{x}) F_{\alpha \beta}(-\omega, \boldsymbol{x})\right) \\
& =\int \frac{d \omega}{2 \pi} \bar{k}^{\mu} \bar{k}^{\nu} A^{\alpha}(\omega, \boldsymbol{x}) A_{\alpha}(-\omega, \boldsymbol{x}) .
\end{aligned}
$$

Armed with these results, it is an easy matter to complete the derivation. The momentum radiated is

$$
\begin{aligned}
K^{\mu} & =\lim _{|\boldsymbol{x}| \rightarrow \infty} \int \frac{d \omega}{2 \pi} \int d \Omega_{2}|\boldsymbol{x}|^{2} n_{\nu} \bar{k}^{\mu} \bar{k}^{\nu} A^{\alpha}(\omega, \boldsymbol{x}) A_{\alpha}(-\omega, \boldsymbol{x}) \\
& =-\frac{1}{(2 \pi)^{3}} \int_{0}^{\infty} d \omega \omega^{2} \int d \Omega_{2} \frac{1}{2 \omega} \bar{k}^{\mu} \tilde{J}^{\alpha}(\bar{k}) \tilde{J}_{\alpha}(-\bar{k}) \\
& =-\frac{1}{(2 \pi)^{3}} \int d^{3} \bar{k} d \bar{k}^{0} \frac{\delta\left(\bar{k}^{0}-|\overline{\boldsymbol{k}}|\right)}{2|\overline{\boldsymbol{k}}|} \bar{k}^{\mu} \tilde{J}^{\alpha}(\bar{k}) \tilde{J}_{\alpha}(-\bar{k}) \\
& =-\int d \Phi(\bar{k}) \bar{k}^{\mu} \tilde{J}^{\alpha}(\bar{k}) \tilde{J}_{\alpha}^{*}(\bar{k}),
\end{aligned}
$$

as expected. In this derivation, we lost manifest Lorentz invariance during the calculation, but we were able to restore it at the end because the observable of interest is Lorentz invariant.

Open Access. This article is distributed under the terms of the Creative Commons Attribution License (CC-BY 4.0), which permits any use, distribution and reproduction in any medium, provided the original author(s) and source are credited. 


\section{References}

[1] LIGO Scientific and Virgo collaborations, Observation of Gravitational Waves from a Binary Black Hole Merger, Phys. Rev. Lett. 116 (2016) 061102 [arXiv:1602.03837] [INSPIRE].

[2] LIGO SCIENTIFIC and VIRGO collaborations, GW151226: Observation of Gravitational Waves from a 22-Solar-Mass Binary Black Hole Coalescence, Phys. Rev. Lett. 116 (2016) 241103 [arXiv: 1606.04855] [INSPIRE].

[3] LIGO ScIEntific and Virgo collaborations, GW170104: Observation of a 50-Solar-Mass Binary Black Hole Coalescence at Redshift 0.2, Phys. Rev. Lett. 118 (2017) 221101 [Erratum ibid. 121 (2018) 129901] [arXiv:1706.01812] [INSPIRE].

[4] LIGO ScIEntific and VIRGo collaborations, GW170814: A Three-Detector Observation of Gravitational Waves from a Binary Black Hole Coalescence, Phys. Rev. Lett. 119 (2017) 141101 [arXiv:1709.09660] [INSPIRE].

[5] LIGO ScIEnTIFIC and VIRGo collaborations, GW17081\%: Observation of Gravitational Waves from a Binary Neutron Star Inspiral, Phys. Rev. Lett. 119 (2017) 161101 [arXiv:1710.05832] [INSPIRE].

[6] A. Buonanno and B.S. Sathyaprakash, Sources of Gravitational Waves: Theory and Observations, pp. 287-346, (2014), arXiv:1410.7832 [INSPIRE].

[7] R. Arnowitt and S. Deser, Quantum Theory of Gravitation: General Formulation and Linearized Theory, Phys. Rev. 113 (1959) 745 [INSPIRE].

[8] R.L. Arnowitt, S. Deser and C.W. Misner, Dynamical Structure and Definition of Energy in General Relativity, Phys. Rev. 116 (1959) 1322 [INSPIRE].

[9] R.L. Arnowitt, S. Deser and C.W. Misner, Canonical variables for general relativity, Phys. Rev. 117 (1960) 1595 [INSPIRE].

[10] R.L. Arnowitt, S. Deser and C.W. Misner, The Dynamics of general relativity, Gen. Rel. Grav. 40 (2008) 1997 [gr-qc/0405109] [INSPIRE].

[11] G. Schäfer and P. Jaranowski, Hamiltonian formulation of general relativity and post-Newtonian dynamics of compact binaries, Living Rev. Rel. 21 (2018) 7 [arXiv: 1805.07240] [INSPIRE].

[12] L. Blanchet, Gravitational Radiation from Post-Newtonian Sources and Inspiralling Compact Binaries, Living Rev. Rel. 17 (2014) 2 [arXiv:1310.1528] [INSPIRE].

[13] A. Buonanno and T. Damour, Effective one-body approach to general relativistic two-body dynamics, Phys. Rev. D 59 (1999) 084006 [gr-qc/9811091] [INSPIRE].

[14] A. Buonanno and T. Damour, Transition from inspiral to plunge in binary black hole coalescences, Phys. Rev. D 62 (2000) 064015 [gr-qc/0001013] [INSPIRE].

[15] T. Damour, Coalescence of two spinning black holes: an effective one-body approach, Phys. Rev. D 64 (2001) 124013 [gr-qc/0103018] [INSPIRE].

[16] T. Damour, P. Jaranowski and G. Schaefer, On the determination of the last stable orbit for circular general relativistic binaries at the third postNewtonian approximation, Phys. Rev. D 62 (2000) 084011 [gr-qc/0005034] [INSPIRE].

[17] F. Pretorius, Evolution of binary black hole spacetimes, Phys. Rev. Lett. 95 (2005) 121101 [gr-qc/0507014] [INSPIRE]. 
[18] F. Pretorius, Binary Black Hole Coalescence, arXiv:0710.1338 [INSPIRE].

[19] W.D. Goldberger and I.Z. Rothstein, An Effective field theory of gravity for extended objects, Phys. Rev. D 73 (2006) 104029 [hep-th/0409156] [INSPIRE].

[20] R.A. Porto, The effective field theorist's approach to gravitational dynamics, Phys. Rept. 633 (2016) 1 [arXiv: 1601.04914] [INSPIRE].

[21] M. Levi, Effective Field Theories of Post-Newtonian Gravity: A comprehensive review, arXiv: 1807.01699 [INSPIRE].

[22] W.D. Goldberger and I.Z. Rothstein, Dissipative effects in the worldline approach to black hole dynamics, Phys. Rev. D 73 (2006) 104030 [hep-th/0511133] [INSPIRE].

[23] W.D. Goldberger and I.Z. Rothstein, Towers of Gravitational Theories, Gen. Rel. Grav. 38 (2006) 1537 [hep-th/0605238] [INSPIRE].

[24] R.A. Porto, Post-Newtonian corrections to the motion of spinning bodies in NRGR, Phys. Rev. D 73 (2006) 104031 [gr-qc/0511061] [INSPIRE].

[25] R.A. Porto and I.Z. Rothstein, The Hyperfine Einstein-Infeld-Hoffmann potential, Phys. Rev. Lett. 97 (2006) 021101 [gr-qc/0604099] [INSPIRE].

[26] R.A. Porto, Absorption effects due to spin in the worldline approach to black hole dynamics, Phys. Rev. D 77 (2008) 064026 [arXiv:0710.5150] [INSPIRE].

[27] R.A. Porto and I.Z. Rothstein, Comment on 'On the next-to-leading order gravitational spin(1)-spin(2) dynamics' by J. Steinhoff et al., arXiv:0712.2032 [INSPIRE].

[28] B. Kol and M. Smolkin, Non-Relativistic Gravitation: From Newton to Einstein and Back, Class. Quant. Grav. 25 (2008) 145011 [arXiv:0712.4116] [INSPIRE].

[29] C.R. Galley and B.L. Hu, Self-force on extreme mass ratio inspirals via curved spacetime effective field theory, Phys. Rev. D 79 (2009) 064002 [arXiv:0801.0900] [INSPIRE].

[30] R.A. Porto and I.Z. Rothstein, Spin(1)Spin(2) Effects in the Motion of Inspiralling Compact Binaries at Third Order in the Post-Newtonian Expansion, Phys. Rev. D 78 (2008) 044012 [Erratum ibid. D 81 (2010) 029904] [arXiv:0802.0720] [INSPIRE].

[31] M. Levi, Next to Leading Order gravitational Spin1-Spin2 coupling with Kaluza-Klein reduction, Phys. Rev. D 82 (2010) 064029 [arXiv:0802.1508] [InSPIRE].

[32] B. Kol, The Delocalized Effective Degrees of Freedom of a Black Hole at Low Frequencies, Gen. Rel. Grav. 40 (2008) 2061 [arXiv:0804.0187] [inSPIRE].

[33] R.A. Porto and I.Z. Rothstein, Next to Leading Order Spin(1)Spin(1) Effects in the Motion of Inspiralling Compact Binaries, Phys. Rev. D 78 (2008) 044013 [Erratum ibid. D 81 (2010) 029905] [arXiv: 0804.0260] [INSPIRE].

[34] J.B. Gilmore and A. Ross, Effective field theory calculation of second post-Newtonian binary dynamics, Phys. Rev. D 78 (2008) 124021 [arXiv:0810.1328] [INSPIRE].

[35] Y.-Z. Chu, The n-body problem in General Relativity up to the second post-Newtonian order from perturbative field theory, Phys. Rev. D 79 (2009) 044031 [arXiv: 0812. 0012] [INSPIRE].

[36] W.D. Goldberger and A. Ross, Gravitational radiative corrections from effective field theory, Phys. Rev. D 81 (2010) 124015 [arXiv: 0912.4254] [InSPIRE].

[37] C.R. Galley and M. Tiglio, Radiation reaction and gravitational waves in the effective field theory approach, Phys. Rev. D 79 (2009) 124027 [arXiv:0903.1122] [INSPIRE]. 
[38] B. Kol and M. Smolkin, Dressing the Post-Newtonian two-body problem and Classical Effective Field Theory, Phys. Rev. D 80 (2009) 124044 [arXiv:0910.5222] [INSPIRE].

[39] R.A. Porto, Next to leading order spin-orbit effects in the motion of inspiralling compact binaries, Class. Quant. Grav. 27 (2010) 205001 [arXiv:1005.5730] [INSPIRE].

[40] M. Levi, Next to Leading Order gravitational Spin-Orbit coupling in an Effective Field Theory approach, Phys. Rev. D 82 (2010) 104004 [arXiv:1006.4139] [INSPIRE].

[41] R.A. Porto, A. Ross and I.Z. Rothstein, Spin induced multipole moments for the gravitational wave flux from binary inspirals to third Post-Newtonian order, JCAP 03 (2011) 009 [arXiv: 1007.1312] [INSPIRE].

[42] B. Kol, M. Levi and M. Smolkin, Comparing space+time decompositions in the post-Newtonian limit, Class. Quant. Grav. 28 (2011) 145021 [arXiv:1011.6024] [InSPIRE].

[43] S. Foffa and R. Sturani, Effective field theory calculation of conservative binary dynamics at third post-Newtonian order, Phys. Rev. D 84 (2011) 044031 [arXiv:1104.1122] [InSPIRE].

[44] M. Levi, Binary dynamics from spin1-spin2 coupling at fourth post-Newtonian order, Phys. Rev. D 85 (2012) 064043 [arXiv:1107.4322] [INSPIRE].

[45] S. Hergt, J. Steinhoff and G. Schaefer, Elimination of the spin supplementary condition in the effective field theory approach to the post-Newtonian approximation, Annals Phys. $\mathbf{3 2 7}$ (2012) 1494 [arXiv: 1110.2094] [INSPIRE].

[46] S. Foffa and R. Sturani, Tail terms in gravitational radiation reaction via effective field theory, Phys. Rev. D 87 (2013) 044056 [arXiv: 1111.5488] [INSPIRE].

[47] A. Ross, Multipole expansion at the level of the action, Phys. Rev. D 85 (2012) 125033 [arXiv: 1202.4750] [INSPIRE].

[48] R.A. Porto, A. Ross and I.Z. Rothstein, Spin induced multipole moments for the gravitational wave amplitude from binary inspirals to 2.5 Post-Newtonian order, JCAP 09 (2012) 028 [arXiv: 1203.2962] [INSPIRE].

[49] C.R. Galley and A.K. Leibovich, Radiation reaction at 3.5 post-Newtonian order in effective field theory, Phys. Rev. D 86 (2012) 044029 [arXiv: 1205.3842] [INSPIRE].

[50] W.D. Goldberger, A. Ross and I.Z. Rothstein, Black hole mass dynamics and renormalization group evolution, Phys. Rev. D 89 (2014) 124033 [arXiv:1211.6095] [INSPIRE].

[51] C.R. Galley and R.A. Porto, Gravitational self-force in the ultra-relativistic limit: the "large- $N$ " expansion, JHEP 11 (2013) 096 [arXiv: 1302.4486] [INSPIRE].

[52] O. Birnholtz, S. Hadar and B. Kol, Theory of post-Newtonian radiation and reaction, Phys. Rev. D 88 (2013) 104037 [arXiv: 1305.6930] [INSPIRE].

[53] S. Chakrabarti, T. Delsate and J. Steinhoff, Effective action and linear response of compact objects in Newtonian gravity, Phys. Rev. D 88 (2013) 084038 [arXiv:1306.5820] [INSPIRE].

[54] S. Foffa and R. Sturani, Effective field theory methods to model compact binaries, Class. Quant. Grav. 31 (2014) 043001 [arXiv:1309.3474] [INSPIRE].

[55] S. Foffa, Gravitating binaries at 5PN in the post-Minkowskian approximation, Phys. Rev. D 89 (2014) 024019 [arXiv:1309.3956] [INSPIRE]. 
[56] M. Levi and J. Steinhoff, Equivalence of ADM Hamiltonian and Effective Field Theory approaches at next-to-next-to-leading order spin1-spin2 coupling of binary inspirals, JCAP 12 (2014) 003 [arXiv: 1408.5762] [INSPIRE].

[57] M. Levi and J. Steinhoff, Leading order finite size effects with spins for inspiralling compact binaries, JHEP 06 (2015) 059 [arXiv:1410.2601] [INSPIRE].

[58] M. Levi and J. Steinhoff, Spinning gravitating objects in the effective field theory in the post-Newtonian scheme, JHEP 09 (2015) 219 [arXiv:1501.04956] [INSPIRE].

[59] M. Levi and J. Steinhoff, Next-to-next-to-leading order gravitational spin-orbit coupling via the effective field theory for spinning objects in the post-Newtonian scheme, JCAP 01 (2016) 011 [arXiv: 1506.05056] [INSPIRE].

[60] M. Levi and J. Steinhoff, Next-to-next-to-leading order gravitational spin-squared potential via the effective field theory for spinning objects in the post-Newtonian scheme, JCAP 01 (2016) 008 [arXiv: 1506.05794] [INSPIRE].

[61] C.R. Galley, A.K. Leibovich, R.A. Porto and A. Ross, Tail effect in gravitational radiation reaction: Time nonlocality and renormalization group evolution, Phys. Rev. D 93 (2016) 124010 [arXiv: 1511.07379] [INSPIRE].

[62] M. Levi and J. Steinhoff, Complete conservative dynamics for inspiralling compact binaries with spins at fourth post-Newtonian order, arXiv:1607.04252 [INSPIRE].

[63] S. Foffa, P. Mastrolia, R. Sturani and C. Sturm, Effective field theory approach to the gravitational two-body dynamics, at fourth post-Newtonian order and quintic in the Newton constant, Phys. Rev. D 95 (2017) 104009 [arXiv: 1612.00482] [INSPIRE].

[64] R.A. Porto and I.Z. Rothstein, Apparent ambiguities in the post-Newtonian expansion for binary systems, Phys. Rev. D 96 (2017) 024062 [arXiv:1703.06433] [INSPIRE].

[65] R.A. Porto, Lamb shift and the gravitational binding energy for binary black holes, Phys. Rev. D 96 (2017) 024063 [arXiv: 1703.06434] [INSPIRE].

[66] M. Levi and J. Steinhoff, EFTofPNG: A package for high precision computation with the Effective Field Theory of Post-Newtonian Gravity, Class. Quant. Grav. 34 (2017) 244001 [arXiv: 1705. 06309] [INSPIRE].

[67] N.T. Maia, C.R. Galley, A.K. Leibovich and R.A. Porto, Radiation reaction for spinning bodies in effective field theory I: Spin-orbit effects, Phys. Rev. D 96 (2017) 084064 [arXiv: 1705.07934] [INSPIRE].

[68] N.T. Maia, C.R. Galley, A.K. Leibovich and R.A. Porto, Radiation reaction for spinning bodies in effective field theory II: Spin-spin effects, Phys. Rev. D 96 (2017) 084065 [arXiv: 1705. 07938] [INSPIRE].

[69] D. Neill and I.Z. Rothstein, Classical Space-Times from the S Matrix, Nucl. Phys. B $\mathbf{8 7 7}$ (2013) 177 [arXiv: 1304.7263] [INSPIRE].

[70] N.E.J. Bjerrum-Bohr, J.F. Donoghue and P. Vanhove, On-shell Techniques and Universal Results in Quantum Gravity, JHEP 02 (2014) 111 [arXiv:1309.0804] [INSPIRE].

[71] N.E.J. Bjerrum-Bohr, B.R. Holstein, L. Planté and P. Vanhove, Graviton-Photon Scattering, Phys. Rev. D 91 (2015) 064008 [arXiv:1410.4148] [INSPIRE]. 
[72] N.E.J. Bjerrum-Bohr, J.F. Donoghue, B.R. Holstein, L. Planté and P. Vanhove, Bending of Light in Quantum Gravity, Phys. Rev. Lett. 114 (2015) 061301 [arXiv:1410.7590] [INSPIRE].

[73] N.E.J. Bjerrum-Bohr, J.F. Donoghue, B.R. Holstein, L. Plante and P. Vanhove, Light-like Scattering in Quantum Gravity, JHEP 11 (2016) 117 [arXiv:1609.07477] [INSPIRE].

[74] F. Cachazo and A. Guevara, Leading Singularities and Classical Gravitational Scattering, arXiv: 1705.10262 [INSPIRE].

[75] A. Guevara, Holomorphic Classical Limit for Spin Effects in Gravitational and Electromagnetic Scattering, arXiv:1706.02314 [INSPIRE].

[76] N.E.J. Bjerrum-Bohr, P.H. Damgaard, G. Festuccia, L. Planté and P. Vanhove, General Relativity from Scattering Amplitudes, Phys. Rev. Lett. 121 (2018) 171601 [arXiv: 1806.04920] [INSPIRE].

[77] J.F. Donoghue, Leading quantum correction to the Newtonian potential, Phys. Rev. Lett. 72 (1994) 2996 [gr-qc/9310024] [INSPIRE].

[78] J.F. Donoghue, General relativity as an effective field theory: The leading quantum corrections, Phys. Rev. D 50 (1994) 3874 [gr-qc/9405057] [INSPIRE].

[79] J.F. Donoghue and T. Torma, On the power counting of loop diagrams in general relativity, Phys. Rev. D 54 (1996) 4963 [hep-th/9602121] [INSPIRE].

[80] J.F. Donoghue, B.R. Holstein, B. Garbrecht and T. Konstandin, Quantum corrections to the Reissner-Nordstrom and Kerr-Newman metrics, Phys. Lett. B 529 (2002) 132 [Erratum ibid. B 612 (2005) 311] [hep-th/0112237] [INSPIRE].

[81] N.E.J. Bjerrum-Bohr, J.F. Donoghue and B.R. Holstein, Quantum corrections to the Schwarzschild and Kerr metrics, Phys. Rev. D 68 (2003) 084005 [Erratum ibid. D 71 (2005) 069904] [hep-th/0211071] [INSPIRE].

[82] N.E.J. Bjerrum-Bohr, J.F. Donoghue and B.R. Holstein, Quantum gravitational corrections to the nonrelativistic scattering potential of two masses, Phys. Rev. D 67 (2003) 084033 [Erratum ibid. D 71 (2005) 069903] [hep-th/0211072] [inSPIRE].

[83] B.R. Holstein and J.F. Donoghue, Classical physics and quantum loops, Phys. Rev. Lett. 93 (2004) 201602 [hep-th/0405239] [INSPIRE].

[84] T. Damour, Gravitational scattering, post-Minkowskian approximation and Effective One-Body theory, Phys. Rev. D 94 (2016) 104015 [arXiv: 1609.00354] [InSPIRE].

[85] T. Damour, High-energy gravitational scattering and the general relativistic two-body problem, Phys. Rev. D 97 (2018) 044038 [arXiv:1710.10599] [INSPIRE].

[86] H. Kawai, D.C. Lewellen and S.H.H. Tye, A Relation Between Tree Amplitudes of Closed and Open Strings, Nucl. Phys. B 269 (1986) 1 [INSPIRE].

[87] Z. Bern, J.J.M. Carrasco and H. Johansson, New Relations for Gauge-Theory Amplitudes, Phys. Rev. D 78 (2008) 085011 [arXiv: 0805. 3993] [INSPIRE].

[88] Z. Bern, J.J.M. Carrasco and H. Johansson, Perturbative Quantum Gravity as a Double Copy of Gauge Theory, Phys. Rev. Lett. 105 (2010) 061602 [arXiv:1004.0476] [INSPIRE].

[89] R. Monteiro, D. O'Connell and C.D. White, Black holes and the double copy, JHEP 12 (2014) 056 [arXiv: 1410.0239] [INSPIRE]. 
[90] A. Luna, R. Monteiro, I. Nicholson, D. O'Connell and C.D. White, The double copy: Bremsstrahlung and accelerating black holes, JHEP 06 (2016) 023 [arXiv: 1603.05737] [INSPIRE].

[91] W.D. Goldberger and A.K. Ridgway, Radiation and the classical double copy for color charges, Phys. Rev. D 95 (2017) 125010 [arXiv:1611.03493] [INSPIRE].

[92] A. Luna et al., Perturbative spacetimes from Yang-Mills theory, JHEP 04 (2017) 069 [arXiv: 1611.07508] [INSPIRE].

[93] A. Luna, R. Monteiro, I. Nicholson and D. O'Connell, Type D Spacetimes and the Weyl Double Copy, arXiv:1810.08183 [INSPIRE].

[94] W.D. Goldberger, S.G. Prabhu and J.O. Thompson, Classical gluon and graviton radiation from the bi-adjoint scalar double copy, Phys. Rev. D 96 (2017) 065009 [arXiv:1705.09263] [INSPIRE].

[95] C.-H. Shen, Gravitational Radiation from Color-Kinematics Duality, JHEP 11 (2018) 162 [arXiv: 1806. 07388] [INSPIRE].

[96] W.D. Goldberger and A.K. Ridgway, Bound states and the classical double copy, Phys. Rev. D 97 (2018) 085019 [arXiv:1711.09493] [INSPIRE].

[97] J. Plefka, J. Steinhoff and W. Wormsbecher, Effective action of dilaton gravity as the classical double copy of Yang-Mills theory, Phys. Rev. D 99 (2019) 024021 [arXiv: 1807.09859] [INSPIRE].

[98] W.D. Goldberger, J. Li and S.G. Prabhu, Spinning particles, axion radiation and the classical double copy, Phys. Rev. D 97 (2018) 105018 [arXiv:1712.09250] [INSPIRE].

[99] J. Li and S.G. Prabhu, Gravitational radiation from the classical spinning double copy, Phys. Rev. D 97 (2018) 105019 [arXiv:1803. 02405] [inSPIRE].

[100] B.R. Holstein and A. Ross, Spin Effects in Long Range Gravitational Scattering, arXiv:0802.0716 [INSPIRE].

[101] C. Cheung, I.Z. Rothstein and M.P. Solon, From Scattering Amplitudes to Classical Potentials in the Post-Minkowskian Expansion, Phys. Rev. Lett. 121 (2018) 251101 [arXiv: 1808. 02489] [INSPIRE].

[102] A. Laddha and A. Sen, Gravity Waves from Soft Theorem in General Dimensions, JHEP 09 (2018) 105 [arXiv: 1801.07719] [INSPIRE].

[103] A. Laddha and A. Sen, Logarithmic Terms in the Soft Expansion in Four Dimensions, JHEP 10 (2018) 056 [arXiv: 1804.09193] [INSPIRE].

[104] B. Sahoo and A. Sen, Classical and Quantum Results on Logarithmic Terms in the Soft Theorem in Four Dimensions, arXiv: 1808.03288 [INSPIRE].

[105] H.A. Lorentz, La théorie élecromagnetique de Maxwell et son application aux corps mouvemants, Arch. Néerl. Sci. Exactes Nat. 25 (1892) 363.

[106] M. Abraham, Prinzipien der Dynamik des Elektrons, Ann. Phys. 10 (1903) 105.

[107] M. Abraham, Theorie der Elektrizität. Vol. II: Elektromagnetische Theorie der Strahlung, Teubner, Leipzig Germany (1904).

[108] M. Abraham, Zur Theorie der Strahlung und des Strahlungsdruckes, Ann. Phys. 14 (1904) 236. 
[109] P.A.M. Dirac, Classical theory of radiating electrons, Proc. Roy. Soc. Lond. A 167 (1938) 148.

[110] L.D. Landau and E.M. Lifshitz, The Classical Theory of Fields, fourth edition, Butterworth-Heinemann, Oxford U.K. (1975), [ISBN:978-0750627689].

[111] A. Higuchi, Radiation reaction in quantum field theory, Phys. Rev. D 66 (2002) 105004 [Erratum ibid. D 69 (2004) 129903] [quant-ph/0208017] [INSPIRE].

[112] C.R. Galley, B.L. Hu and S.-Y. Lin, Electromagnetic and gravitational self-force on a relativistic particle from quantum fields in curved space, Phys. Rev. D 74 (2006) 024017 [gr-qc/0603099] [INSPIRE].

[113] C.R. Galley, A.K. Leibovich and I.Z. Rothstein, Finite size corrections to the radiation reaction force in classical electrodynamics, Phys. Rev. Lett. 105 (2010) 094802 [arXiv: 1005.2617] [INSPIRE].

[114] O. Birnholtz, S. Hadar and B. Kol, Theory of post-Newtonian radiation and reaction, Phys. Rev. D 88 (2013) 104037 [arXiv: 1305.6930] [InSPIRE].

[115] O. Birnholtz, S. Hadar and B. Kol, Radiation reaction at the level of the action, Int. J. Mod. Phys. A 29 (2014) 1450132 [arXiv:1402.2610] [INSPIRE].

[116] O. Birnholtz, Comments on initial conditions for the Abraham-Lorentz(-Dirac) equation, Int. J. Mod. Phys. A 30 (2015) 1550011 [arXiv:1410.5871] [INSPIRE].

[117] M.H. Al-Hashimi and U.J. Wiese, Minimal Position-Velocity Uncertainty Wave Packets in Relativistic and Non-relativistic Quantum Mechanics, Annals Phys. 324 (2009) 2599 [arXiv:0907.5178] [INSPIRE].

[118] D. Amati, M. Ciafaloni and G. Veneziano, Superstring Collisions at Planckian Energies, Phys. Lett. B 197 (1987) 81 [inSPIRE].

[119] G. 't Hooft, Graviton Dominance in Ultrahigh-Energy Scattering, Phys. Lett. B 198 (1987) 61 [INSPIRE].

[120] I.J. Muzinich and M. Soldate, High-Energy Unitarity of Gravitation and Strings, Phys. Rev. D 37 (1988) 359 [INSPIRE].

[121] D. Amati, M. Ciafaloni and G. Veneziano, Classical and Quantum Gravity Effects from Planckian Energy Superstring Collisions, Int. J. Mod. Phys. A 3 (1988) 1615 [inSPIRE].

[122] D. Amati, M. Ciafaloni and G. Veneziano, Higher Order Gravitational Deflection and Soft Bremsstrahlung in Planckian Energy Superstring Collisions, Nucl. Phys. B 347 (1990) 550 [INSPIRE].

[123] D. Amati, M. Ciafaloni and G. Veneziano, Planckian scattering beyond the semiclassical approximation, Phys. Lett. B 289 (1992) 87 [INSPIRE].

[124] D.N. Kabat and M. Ortiz, Eikonal quantum gravity and Planckian scattering, Nucl. Phys. B 388 (1992) 570 [hep-th/9203082] [INSPIRE].

[125] D. Amati, M. Ciafaloni and G. Veneziano, Effective action and all order gravitational eikonal at Planckian energies, Nucl. Phys. B 403 (1993) 707 [InSPIRE].

[126] I.J. Muzinich and S. Vokos, Long range forces in quantum gravity, Phys. Rev. D 52 (1995) 3472 [hep-th/9501083] [INSPIRE].

[127] G. D'Appollonio, P. Di Vecchia, R. Russo and G. Veneziano, High-energy string-brane scattering: Leading eikonal and beyond, JHEP 11 (2010) 100 [arXiv:1008.4773] [INSPIRE]. 
[128] S. Melville, S.G. Naculich, H.J. Schnitzer and C.D. White, Wilson line approach to gravity in the high energy limit, Phys. Rev. D 89 (2014) 025009 [arXiv:1306.6019] [InSPIRE].

[129] R. Akhoury, R. Saotome and G. Sterman, High Energy Scattering in Perturbative Quantum Gravity at Next to Leading Power, arXiv:1308.5204 [INSPIRE].

[130] G. D'Appollonio, P. Di Vecchia, R. Russo and G. Veneziano, Regge behavior saves String Theory from causality violations, JHEP 05 (2015) 144 [arXiv: 1502.01254] [INSPIRE].

[131] M. Ciafaloni, D. Colferai and G. Veneziano, Emerging Hawking-Like Radiation from Gravitational Bremsstrahlung Beyond the Planck Scale, Phys. Rev. Lett. 115 (2015) 171301 [arXiv: 1505. 06619] [INSPIRE].

[132] G. D'Appollonio, P. Di Vecchia, R. Russo and G. Veneziano, A microscopic description of absorption in high-energy string-brane collisions, JHEP 03 (2016) 030 [arXiv: 1510.03837] [INSPIRE].

[133] M. Ciafaloni, D. Colferai, F. Coradeschi and G. Veneziano, Unified limiting form of graviton radiation at extreme energies, Phys. Rev. D 93 (2016) 044052 [arXiv:1512.00281] [INSPIRE].

[134] A. Luna, S. Melville, S.G. Naculich and C.D. White, Next-to-soft corrections to high energy scattering in QCD and gravity, JHEP 01 (2017) 052 [arXiv: 1611.02172] [INSPIRE].

[135] A.K. Collado, P. Di Vecchia, R. Russo and S. Thomas, The subleading eikonal in supergravity theories, JHEP 10 (2018) 038 [arXiv: 1807.04588] [INSPIRE].

[136] J. Sucher, Two photon exchange force in scalar quantum electrodynamics: The Asymptotic story, Phys. Rev. D 49 (1994) 4284 [INSPIRE].

[137] A. Luna, I. Nicholson, D. O'Connell and C.D. White, Inelastic Black Hole Scattering from Charged Scalar Amplitudes, JHEP 03 (2018) 044 [arXiv:1711.03901] [INSPIRE].

[138] J.D. Jackson, Classical Electrodynamics, third edition, Wiley, New York U.S.A. (1998) [ISBN:978-0471309321].

[139] J. Ellis, TikZ-Feynman: Feynman diagrams with TikZ, Comput. Phys. Commun. 210 (2017) 103 [arXiv: 1601.05437] [INSPIRE].

[140] J. Schwinger, L.L. DeRaad Jr., K.A. Milton and W.Y. Tsai, Classical Electrodynamics, Perseus, New York U.S.A. (1998) [ISBN:978-0738200569]. 